\title{
DISCLAIMER
}

This report :-s prepared as an account of work sponsured by an agency of the 1 inited States

UCRL- -53768

Government. Neither the United States Government nor any ageney thereof. now any of their emplovees, makes any wirranty, express or implied, or assumes any legal hathili, ir responsibility for the accuracs, completeness, of usefolness of any information, appatatis. product. of process disclosed. or represen!s that its use would not infrange prwately ounded rights Reference herein 10 any speatic commercial product, process. or service by trade natsic. trademerk. manufacturer, or otherwise does not necessarily constilute or mply its endursernent, recommendation, or favoring by the United States (ivernment of any angency ahereuf The views and opinions of asuthors expressed hercin do not uecessiaris. statc or reflect those of the United States Government or any agchey thereor

\section{Electrical Conductivity and Equation of State of Liquid Nitrogen, Oxygen, Benzene, and 1-Butene Shocked to $60 \mathrm{GPa}$}

Douglas Cummings Hamilton

(Ph.D. Thesis)

\section{Manuscript date: October 8, 1986}

\section{LAWRENCE LIVERMORE NATIONAL LABORATORY University of California - Livermore, California - 94550}


ELECTRICAL COHDUCTIVITY AND EQUATION OF STATE. OF LIQUID NITROGEN, OXYGEN, BENZENE, AND 1-BUTENE SHOCKFD TO 50 GPa

\section{By}

Douglas Cummings Hamilton

\section{DISSERTATION}

Submitted in partial satisfaction of the requirements for the degree of DOCTOR OF PHILOSOPHY

iิn

ENGINEERING-APPLIED SCIENCE

in the

GRADUATE DIVISION

of the

UNIVERSITY OF CALIFORNIA

MAVIS

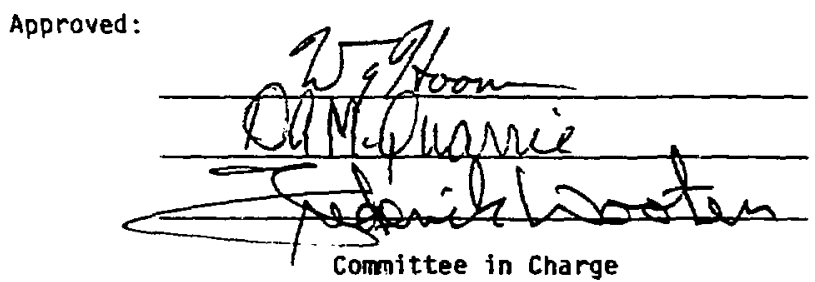

198 
Douglas Cummings Hamitton

September 1986

Applied Scjence

\begin{abstract}
Electrical Conductivity and Equation of State of Liquid Nitrogen, Oxygen, Benzene, and 1-Butene Shocked to $60 \mathrm{GPa}$
\end{abstract}

ABSTRAC 1

Measurements are reported for the electrical conductivity of licuid nitrogen $\left(\mathrm{N}_{2}\right)$, oxygen $\left(\mathrm{O}_{2}\right)$ and benzene $\left(\mathrm{C}_{6} \mathrm{H}_{6}\right)$, and Hugoniot equation of state of liquid 1-butene $\left(\mathrm{C}_{4} \mathrm{H}_{8}\right)$ under shock compressed conditions. The conductivity data span $7 \times 10^{-4}-7 \times 10^{7} \Omega^{-1} \mathrm{~cm}^{-1}$ over a dynamic pressure range 18.1 - 67.5 GPa and are discussed in terms of amorphous semiconduction models which include such transport phenomena as hopping, percolation, pseudogaps, and metallization. Excellent agreement is found between the equation-of-state measurements, which span a dynamic pressure range 12.3 - 53.8 $\mathrm{GPa}$, and Ree's calculated values which assume a 2-phase mixture consisting of molecular hydrogen and carbon in a dense diamondlike phase. There is a 2-1/2 fold increase in the thermal pressure contribution over a less dense, stoichiometrically equivalent liquid.

Chairman of Dissertation Committee: Professor W. G. Hoover Department of Applied Science 


\section{ACKNOWLEDGHENTS}

The author is grateful to Or. W. J. Nellis, Professor W. G. Hoover and $\mathrm{Mr}$. A. C. Mitchell for their encouragement, many thoughtful discussions and experimental insights. A special thanks is given to the staff of H-Division and the Department of Applied Science for providing a stimulating environment conducive to completing this work. The author expresses his appreciation to the personnel of the machine and electronic shops, associated with the two-stage gun, for passing along some of their skills. The author also thanks Ms. R. Jensen for willingly providing secretarial help which was crucial in the completion of this manuscript. Finally, the author recognizes the importance of Ms. K. J. Anderson, a goed friend who has endured and supported this enterprise from the beginning. 
Contents

Page

Chapter I Introduction ....................... . 1

1.7 General Comments .............. 1

1.2 Nitrogen, Oxygen and the Hydrocarbons: Motivation

for Inquiry ................. 3

1.3 Nitrogen, Oxygen and the Hydrocarbons: Recent

Experimental Results ............. 5

1.4 Information Gained From Electrical Conductivity

Measurements ................. 8

Chapter 2 Properties of the Liquid State............. . 13

2.1 Phenomenological Description of a Dense Fluid... 13

2.2 Theoretical Treatment of "Simple" Fluids . . . . 17

2.3 Mixtures .................... 26

Chapter 3 Shock Waves ....................... 30

3.1 Introduction ................ 30

3.2 1-D Elastic Bead Hodel .............. 31

3.3 Sh.ck Wave Formation .............. 35

3.4 Hugoniot Relations .............. 45

3.5 Distended Haterials .............. 50

3.6 Shock Impedarice Matching . . . . . . . . . . 52 
Contents (Continued)

Page

Chapter 4 Experimental Method ............ 57

4.1 Introduction ............... 57

4.2 Two-Stage Light-Gas Gun . . . . . . . . . 57

4.3 Targets ................61

4.4 Diagnostics............... 69

4.5 Sample Preparation and Condensation....... 81

4.6 Temperature Control ...........86

4.7 Mass Velocities and Impedance Matching ......88

4.8 Summary of Experimental Procedure . . . . . . . 93

Chapter 5 Experimental Results . . . . . . . . . . . . 94

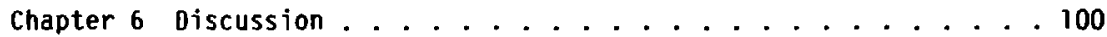

6.1 1-Butene Equation of State ........ 100

6.2 Nitrogen Electrical Conductivity ....... 103

6.3 Oxygen Electrical Conductivity ........ 118

6.4 Benzene Electrical Conductivity........ 122

Appendix A Error Analysis for Electrical Conductivity . . . . . 133

Appendix B Reference Energy for 1-Butene . . . . . . . . 136 
Contents (Continued)

Page

Appendix C Signal Shape for a Constant-Current Circuit ..... 139

Appendix 0 Target Parameters ............... 142

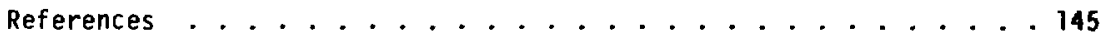




\section{Tables}

Number

Page

4.1 Fitting Parameters for the Liquid/Vapor Saturation Curves . 84

4.2 Initial Sample Densities . . . . . . . . . . . . . . 86

4.3 $U_{p}-U_{s}$ Parameters Used in Shock Impedance Analysis . . . . . 89

5.1 Hugoniot Data for Liquid 1-8utene . . . . . . . . . . . . 94

5.2 Electrical Conductivity of Shock Compressed Benzene, Nitrogen, and oxygen .................. 95

6.1 Dissociation Products of 1 -Butene at $\mathrm{T}=5800 \mathrm{~K}$ and

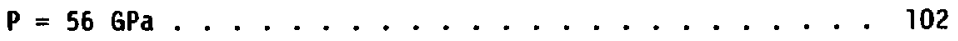

$6.2^{*}$ Degree of compression of Benzene and 0iamond ....... 129

0.1 Baseplate and Impactor Densities, Targe Dimensions, Electrode Materials, and Cell Constants . . . . . . . 144 
Figures

Number

Page

1.1 Hugoniot of liquid nitrogen in $\mathrm{P}-\mathrm{V}$ space $\ldots \ldots$

1.2 Hugoniot of liquid oxygen in P-V space . . . . . . . . 8

1.3 Hugoniot of liquid benzene in $\mathrm{P}-\mathrm{V}$ space........ .99

2.1 Volume response to cooling of a hypothetical material . . 14

2.2 Potentials frequently used in calculating properties if the liquid state ................. 22

3.1 Bead model used to illustrate basic shock wave concepts . . 31

3.2 Velocity history of the first bead ........... 33

$3.3 x-t$ diagram showing the trajectory of each bead ...... 33

3.4 Hugoniot in $\mathrm{P}-\mathrm{Y}$ space for the bead model . . . . . . . . 35

3.5 Shack wave formation ................. 41

3.6 Equation of state for a hypothetical material showing relative positions of the Hugoniot and adiabats for two values of the entropy................. 49

3.? Hugoniots for two similar materials with differing starting densities................... 52

3.8 Impedance matching the impact of two dissimilar materials in $U_{p}-P$ space . . . . . . . . . . . . . . . . . . 54

3.9 Side rarefactions originating at a release point . . . . 56

4.1 Layout of the two-stage light-gas gun and target chamber . 58

4.2 Typical flash $x$-ray records . . . . . . . . . . . 60 
Figures (Continued)

Number

Page

4.3 Cross sectional view of standard target used for benzene conductivity measurements . . . . . . . . . . . . 62

4.4 Cross sectional view of cryogenic target used for 1-butene equation of state, and oxygen and nitrogen conductivity measurements ..................... 63

4.5 Exploded view of electrode sandwich . . . . . . . . . . 64

4.6 Photograph of electrode foils enlarged $4 \mathrm{X} \ldots \ldots 5$

4.7 Pin and thermocouple positions for an equation-of-state measurement . . . . . . . . . . . . . . . .

4.B Equivalent electrical circuits for (a) constant-voltage and (b) constant-current voltage measurements . . . . . . .

4.9 Voltage deflection for a liquid nitrogen conductivity experiment using a constant-voltage circuit . . . . . .

4.10 Current pulser showing capacitor/switching circuit and interlock used in a constant-current conductivity experiment . . . . . . . . . . . . . . . . .

4.11 Essential electrical elements of a low resistance measurement

4.12 Voltage deflection for a liquid nitrogen conductivity experiment using a constant-current circuit 
Figures (Continued)

Number

Page

4.13 Complete elextronic diagnostic system for electrical conductivity experiments ...........

4.14 Typical Tektronix 519 record of pin signals from the first and second plane, and timing fiducial for an equationof-state experiment ..............

4.15 Sample preparation system used for benzene conductivity experiment ................... 82

4.76 Sample condensation system for cryogenic samples . . . . 83

4.17 Temperature control system used for liquid 1-tutene sample. 87

4.18 Impedance matching considerations in an equation-of-state experiment ................ 90

$5.1 \quad 1$-Butene Hugoniot data in $U_{p}-P$ space . . . . . . . 96

5.2 Benzere electrica? conductivity data as a function of shock pressure ................

5.3 Nitrogen electrical conductivity data and Ross' calculated fractional dissociation as a function of shock pressure.

5.4 0xygen electrical conductivity date as a funct: on of shock

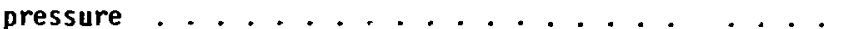

6.1 Comparison of the density of states for (a) crystalline and (b) amorphous semiconductors 


\section{Figures (Continued)}

Nuntier

6.2 The formation of a psuedc gap in the density of states for increasing interatomic separations . . . . . . . 112

6.3 Arrhenius plot of electrical conductivity for shock compressed liquid nitrogen ............. 114

6.4 o-T-P diagram for a hypothetical metallic vapor...... 115

6.5 Dependence of electrical conductivity of shock compressed

liquid nitrogen on the dissociation product separation . 116

6.6 Arrhenius plot of electrical conductivity for shock compressed liquid oxygen ................

6.7 Schematic representation of temperature dependence for carrier concentration and mobility ........... 120

6.8 Arrhenius plot of electrical conductivity for shock compressed liquid benzene . . . . . . . . . . . . 127

6.9 Geometry factor of the resistivity as a function of the volume fraction of diamond ............... 131

B.1 Determining the reference state fur 1-butene . . . . . 136

C. 1 Comparison of signal record with calculated shape assuming constant and reduced fringing field correction .... . 740

D. 1 Target dimensions .................. 143 


\section{CHAPTER 1. INTRODUCTION}

\subsection{General Comments}

In our era, the study of condensed matter physics has expanded and shifted its emphasis to enrompass thermodynamic conditions of extreme temperatures and pressures. Very high pressures (millions of atmospheres) and temperatures (tens of thousands of degrees Kelvin) can be achieved through shock wave-induced dynamic compression. The experimental lifetime is, typically, between $10^{-9}$ and $10^{-6}$ seconds. The significant achievements in obtaining the properties during this transient phenomena have required new measurement techniques and the development of appropriate instrumentation.

The thermodynamic properties of a simple material in equilibrium are determined by a function relating three variables (e.g. pressure, volume and temperature) called the equation of state (EOS). The EOS of a material in its explicit form can be described as a surface in 3-space. Reversibily changing the state of a sample is equivalent to traversing some trajectory located on the surface. Only the initial and final states are in equilbrium, and hence lie on the surface, for a shockcompressed sample. Plotting an extensive and compact mesh of trajectories is desirable if the surface is to be accurately characterized. Trajectories invariably diverge as we sample the more extreme regions of the surface. This, in turn, places a greater burden on models used in describing the material. More often than not, models come equipped with a sufficient supply of adjustable parameters. This is partly a 
consequence of reducing the formidable problen of treating dense, hot materials, by way of modest approxination, into a manageable task. The rub is that several models, based on differing premises. can successfully describe the same trajectory. Uniqueness is restored when errors in premises are revealed as the models venture to enbrace a larger class of trajectories or attempt to predict other properties of the material, such as its transport coefficients. Theoretical approximations necessary in calculating the EOS of a material are minimal compared to those necessary in predicting transport phenomena. This, in itself, justifies an interest in determining the electrical conductivity of shock compressed materials.

Under nomal conditions, the materials investigated in this thesis are excellent insulators. This is due to extremely low concentrations of free charge carriers in addition to the great difficulty carriers experience moving from one location to the next. It is a safe prediction that even the best insulators become good conductors if compressed sufficiently. In a shock compression process the material is also subject to large increases in temperature which can initiate ionization in insulators, excite current carriers across energy gaps in semiconductors, and dissociate complex molecules. Dissociation may allow the constituents to regroup in a denser packing arrangement. Such a transition is expected to accommodate an internal electronic reorganization and may ultimately lead to metalization.

The objective of this endeavor is to investigate the effects of dynamic compression (up to 3.5 fold over initial densities and 
temperatures in excess of $10^{4} \mathrm{~K}$ ) on the electrical conductivity of liquid nitrogen $\left(\mathrm{N}_{2}\right)$, oxygen $\left(\mathrm{O}_{2}\right)$ and benzene $\left(\mathrm{C}_{6} \mathrm{H}_{6}\right)$ and the Hugoniot EOS of liquid 3 -butene $\left(\mathrm{CH}_{2}=\mathrm{CH}-\mathrm{CH}_{2}-\mathrm{CH}_{3}\right)$. The following section examines the issues which motivate the interest in these fluids. Section 1.3 cites recent experimental measurements performed on these fluids and also provides additional motivation for this work. Section 1.4 reviews and interprets other electrical conductivity measurements that have been performed on shock-compressed materials in the last 20-25 years and examines what information might be gained from these experinents. Chapter 2 deals with the properties of liquids. It begins with a discussion of the essential properties which distinguish a liquid from the gaseous and solid phases, followed by a review of theoretical treatments of dense fluids which have successfully predicted the shock-compressed state of these liquids when compared with experimental results. Chapter 3 deals with basic principles of shock wave physics with emphasis on areas relevant in the determination of the final thermodynamic state of the samples. Chapter 4 covers experimental apparatus and procedure. The first part of Chapter 5 presents the data and the second part covers data reduction techniques (with examples) and results. Chapter 6 concludes with interpretive aspects of charge transport in the disordered final state of these dense, hot fluids.

\subsection{Nitrogen, Oxygen and the Hydrocarbons: Motivation for Inquiry}

A knowledge of the properties of liquid nitrogen, oxygen and the hydrocarbons at high densities and temperatures has three primary applications: condensed matter physics, detonation products and planetary 
modeling. The atomic species forming the fluids $\mathrm{H}_{2}, \mathrm{O}_{2}$ and the hydrocarbons (HCS) are the primary constituents of condensed high explosives. The conditions of detonation ( $P=1-100 \mathrm{GPa}$ and $\mathrm{T}=$ $1,000-4,000 \mathrm{~K}$ ) are similar to the high pressures and temperatures realized in a shock wave experiment. Developing models which are suitable in ascertaining detonation behavior of condensed explosives requires detailed knowledge of the individual (often reactive) constituents. ${ }^{1-3}$ These elements are also represented to significant extent on a planetary scale. ${ }^{4,5}$ Jupiter and Saturn are thought to be composed of three layers with the metallic form of hydrogen representing one of the primary components in the middle layer. The thermodynamic state of a small volume element of fluid, constituting the atmosphere of an outer planet, is equivalent to isentropically compressing the material when starting from a cold, dilute phase. The large masses of these planets create large gravitationally-induced, compressive forces which result in large interior pressures. The calculated EOS of these zases, for typical interior pressures $(200-500 \mathrm{GFa})$, gives isentropic temperatures on the order of $10,000 \mathrm{~K}$. These conditions can be sampled on a laboratory scale by shock compressing the liquid phases of these planetary materiais. Hitrogen, in either its molecular or atomic form or as ammonia, is belfeved to be a primary constituent of the planets Uranus and Neptune by virtue of its relatively high cosmological abundance. The importance of knowing the properties of these fluids, in light of the information being gathered at this time by space probes, cannot be overstated. For example, the high temperatures and densities of the planetary interiors of these outer planets may be sufficient to allow metallic forms of nitrogen, which would be necessary in order to invoke 'dynamo' arguments, 
to explain the existence of magnetic fields. The success of a planetary model depends on a sound understanding of the state of the material composition in terms of EOS and other thermodynamic and transport properties under extreme conditions.

\subsection{Nitragen, Oxygen and the Hydrocarbons: Recent Experinental Results}

Recent dynamic experiments indicate that liquid nitrogen undergoes molecular dissociation, thus forming a two-component mixture of monatomic and diatomic nitrogen. ${ }^{6,7,8}$ This is evidenced by an increase in the shock wave compressibility, beginning at shock pressures of $30 \mathrm{GPa}$, and is attributed to the combined effects of shock compression and heating (Fig. 1.1). Below $30 \mathrm{GPa}$, nitrogen appears diatonic. Support for this claim is based on excellent agreement between Hugoniot data and the theoretical P-V curve which uses an intermolecular pair potential derived from an argon potential by corresponding states scaling. ${ }^{9}$ Details of this model will be described in Chapter 2. LeSar and co-workers ${ }^{10}$ and, more recentiy, Reichlin and co-workers ${ }^{11}$ have performed optical measurements on solid nitrogen using a diamond-anvil cell. Reichlin and co-workers ma inta in that nitrogen retains its diatomic character to 130 GPa. Dynamic EOS measurements, temperature measurements of Radousky and co-workers, ${ }^{12}$ and electrical conductivity results of this thes is all seem to support the claim for dissociation at high temperatures. A substantial degree of electronic excitation or ionization is required to account for the large conductivities reported in this thesis; an amount which greatly exceeds the degree expected based on ratios of the ionization energy of dilute $\mathrm{N}_{2}$ fluid to the temperatures accompanying 


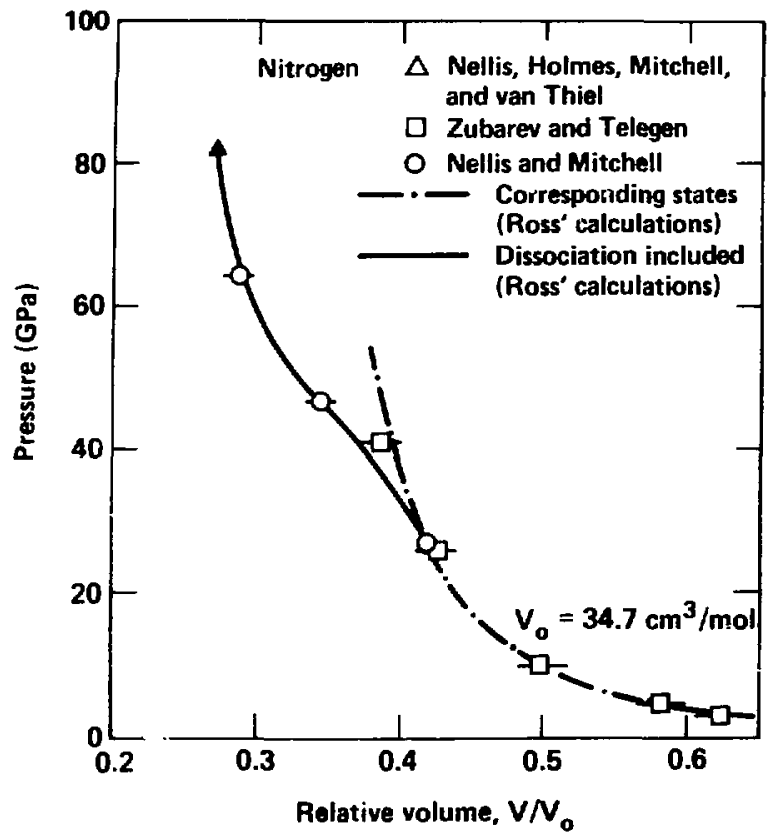

Fig. 1.1 Hugoniot of liquid nitrogen in P-V space. (Refs. 6-8).

the shock compressed state. Extension of the "molecular" model of Ross beyond $30 \mathrm{GPa}$ overestimates the pressure and temperature, which suggests an increasing importance in other energy absorbing mechanisms. Agreement is reestablished between experimental results and theory when allowance is made in the theory fur molecular dissociation with a density-dependent dissociation energy. 13 The high temperatures accompanying the irreversible shock process will also contribute to the relative stability of the monatomic phase. This added feature, whoch distinguishes static from dynamic compression processes, may account for the observed discrepancy regarding the issue of dissociation. Dissociatioi may allow for the redistribution of atoms into a more compact structure. This is 
one interpretation accounting for the softening in the Hugoniot (for instance, a sudden increase in the compressibility). McMahan and LeSar have done calculations on the stability of diamond structure and simple cubic solid phases of monatomic nitrogen and predict a remarkably stable simple cubic phase at $77 \mathrm{GPa}$ which better resembles the structure of other Group $V$ elements such as phosphorous or arsenic. ${ }^{14}$ If this were the case, the initial triple-bonding of molecular nitrogen with its sole nearest neighbor would transform into a structure where the atoms are incorporated into a two-dimensional network of puckered six-fold rings, and the bonding between the three-fold co-ordinated atoms would be mainly covalent.

Hugoniot data of Nellis and Mitchell ${ }^{7}$ and Wackerle, Seita, and Jamieson, ${ }^{15}$ for liquid oxygen are presented in Fig. 1.2. The theoretical curve from Ross is based on the corresponding states scaling of an argon potential and includes internal degrees of freedom such as molecular rotation, vibration and electronic excitation. The lower electronic Energy level; for molecular oxygen are more accessible to population for the temperatures common in these experiments than was the case for molecular nitrogen. Also note the qualitative difference in the Hugoniots of nitrogen and oxygen regarding the absence of any distinct phase transition region for oxygen.

The Hugoniot EOS data of Nellis and co-workers ${ }^{16}$ and Dick ${ }^{17}$ for liquid benzene are presented in Fig. 1.3. A transition region is clearly defined at a pressure of $13 \mathrm{GPa}$, indicating a decomposition phenomenon similar to nitrogen. Yakusheva and co-workers performed time-resolved 


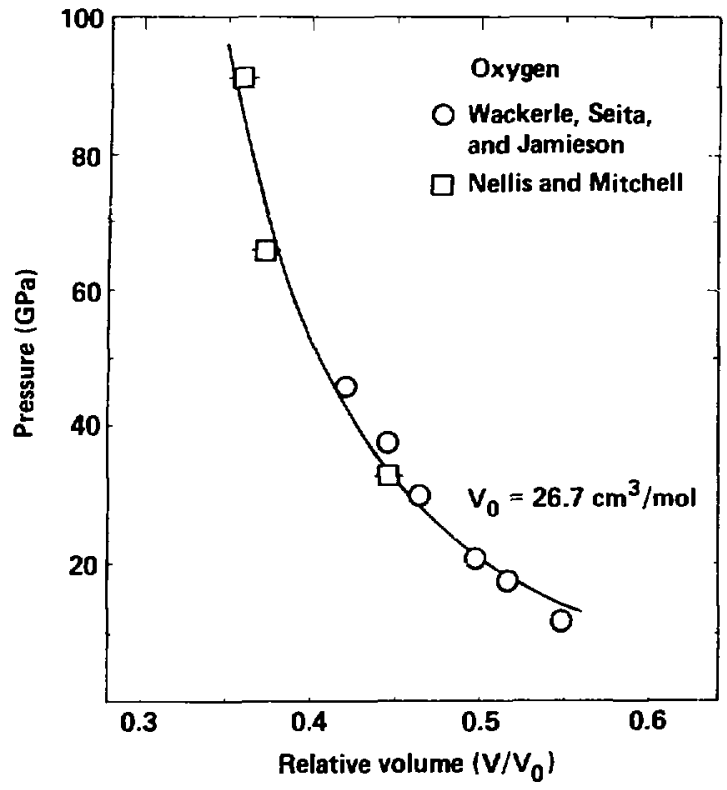

Fig. 1.2 Hugoniot of liquid oxygen in P-V space. Curve is best linear fit through $U_{p}-U_{s}$ data (Ref. 2 and 15).

opacity measurements on shock compressed liquid benzene. ${ }^{18}$ They concluded that benzene irreversibly decomposes to form free carbon particles for dynamic pressures in excess of $13.5 \mathrm{GPa}$. The delay in the onset of opacity was observed to depend on shock magnitude. Higher compressions would correspond to higher temperatures, which they claim would increase the pyrolysis reaction rate and also determine the degree of complexity of the final composition.

\subsection{Information Gained From Electrical Conductivity Measurements}

Methods based on ine use of shock waves offer the unique possibility 


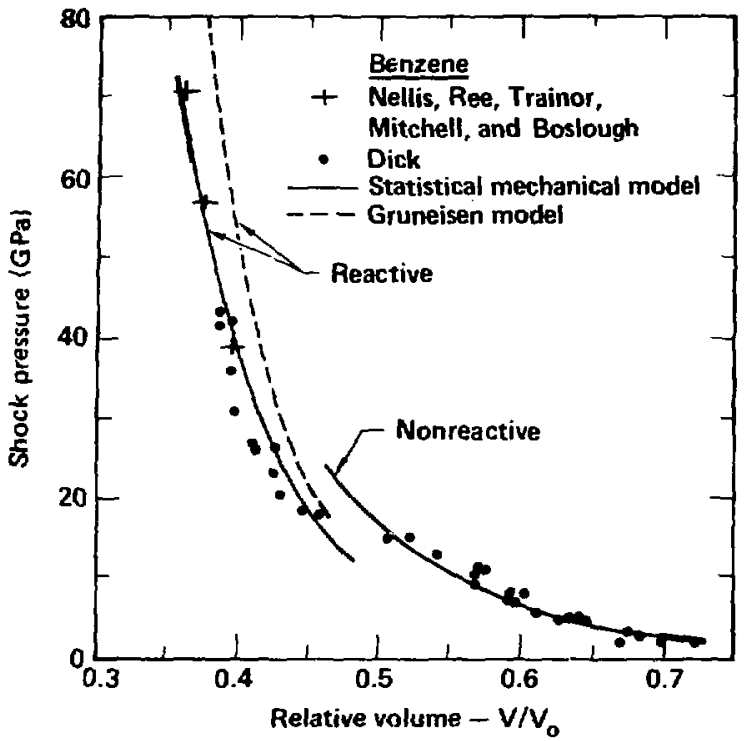

Fig. 1.3 Hugoniot of liquid benzene in P-V space. (Refs. 16 and 17).

of investigating rapid (nanoseconds) physical and chemical processes taking place in condensed materials after a nearly discontinuous change in the pressure and temperature. Two such reactions include phase transitions and insulator-metal transitions. The small compression time at the shock wave front makes it possible to study extreme states of materials which cannot be studied by: other means. The nature of a dynamic experiment alleviates any limitations due to material strengths which often beset static compression experiments. On the other hand, the short time-scale of a dynamic experiment and the necessity of greater distances between experiment and experimenter increases the complexity of the measuring apparatus. The "active" method is used by this author and is based on the probing of a substance by an electric field under pulsed conditions. It is distinguished from the "passive" method which includes 
optical methods and electrical polarization by relying on the shockinduced generation of electromagnetic radiation or an electrical field. The apparatus will be described in greater detail in Chapter 4.

Ershov used the "active" method and reviewed issues concerning charge transport in detonation products and demonstrated the non-trivial problems involved with interpretation. ${ }^{19}$ One of these issues concerns the existence of thermodynamic equilibrium in complex mixtures and will be addressed further in Chapter 3 . In the early sixties Al'tshuler, Kuleshova, and Pavlovski, measured the electricai conductivity of shockcompressed $\mathrm{NaCl} .20$ These early measurements were followed by Balchan and Orickamer, ${ }^{21}$ Kormer and co-workers, ${ }^{22}$ Ahrens ${ }^{23}$ and others. ${ }^{24-31}$ Solids Under Pressure contains several papers which examine, on a semiconductor approach, the parametric influence of pressure on band gaps and insulatormetal transitions. ${ }^{32}$ In Physics of Solids at High Pressures, Fritzsche introduces the connection between impurity conduction and pressure-induced changes in overlap between neighboring impurity states. ${ }^{33}$ A more recent contribution in this field can be found in a monograph by Drickamer and Frank. They jive an extensive account of the pressure effects on the electronic properties of solids and their chemical and physical consequences. ${ }^{34}$ 0tiner references, specific to the development of this thesis, will be cited as needed.

One of the major goals of this thesis is to interpret the experimental results. This will involve connecting the measured values and their thermodynamic state with models which describe successfully the bulk electric properties of disordered, condensed materials. The 
description utilizes concepts and terminology which have proven useful in solid state physics such as mobility and density of states. Typicaliy, these ideas are associated with models requiring an orderly arrangement of atoms in a lattice. A highly ordered (long-range) structure most likely does not occur for the final state of an irreversible shockcompression process. Shock waves are known to create dislocations and induce other energy-increasing imperfections in a p. sshocked perfect crystal lattice like single-crystal diamond.

The unavoidable similarities in optical and electronic properties of amorphous and liquid semiconductors, with those of the crystalline state, set the stage for a description which relies more on an atom's immediate environment and less on long-range order. This approach is still in the development stage, especially with reference to shockcompressed materials. An extensive coverage of this subject can be found in Refs. 35-49.

Theoretical predictions on the shock-compressed state of liquid $i$-butene, based on a statistical mechanical treatment of mixtures, will be compared with EOS data. Measurements were previously made on a stoichiometrically equivalent liquid with an initial density $30 \%$ higher than liquid 1 -butene. So, a rigcrous test for the theory will be its ability to predict accurately the final thermodynamic state of either liquid. For these reasons the author performed the first reported electrical conductivity measurements of shock-compressed liquid nitrogen $\left(N_{2}\right)$, oxygen $\left(\mathrm{O}_{2}\right)$, and benzene $\left(\mathrm{C}_{6} \mathrm{H}_{6}\right)$, and EOS (shock velocity) 
measurements on liquid 1-butene $\left(\mathrm{CH}_{2}=\mathrm{CH}-\mathrm{CH}_{2}-\mathrm{CH}_{3}\right)$ in the dynamic pressure range 12-60 GPa. 
CHAPTER 2. PROPERTIES OF THE LIQUID STATE

\subsection{Phenomenological Description of a Dense Fluid}

The properties that distinguish liquids from gases and solids can be demonstrated effectively by performing a thought experinent involvifig the quenching of a dilute gas contained in some volume at one atmosphere pressure. In the dilute gas phase the "point-particles" are unconstrained by any particle-particle correlation. The nature of the pressire and energy is predominantly kinetic and the motion of the molecules are nearly straight-line trajectories which are punctuated occasionally by sharp deflections due to intermediate collisions. The phase is fluid; offering no resistance to shear stresses. Large changes in efther pressure or temperature result in large changes in yolume (that is, high compressibility). The proportionally large anount of time an atom sxperiences free of intermolecular interaction, as compared to the total time between collisions, motivates a density expansion approach to describe small deviations from the behavior of a classical, ideal gas.

As the gas is cooled, thus lowering the average kinetic energy of the system, a temperature will be reached $\left(T_{B}\right)$ where there will be a discontinuous change in the volume (Fig. 2.1). For most fluids this is condensation to the liquid state. The phase is still fluid but now occupies a well defined volume whose shape is determined by the container. The volume change accompanying a change in pressure or temperature is several orders of magnitude less than the changes accompanying the dilute yas phase. For most liquids 100 kbar pressures 


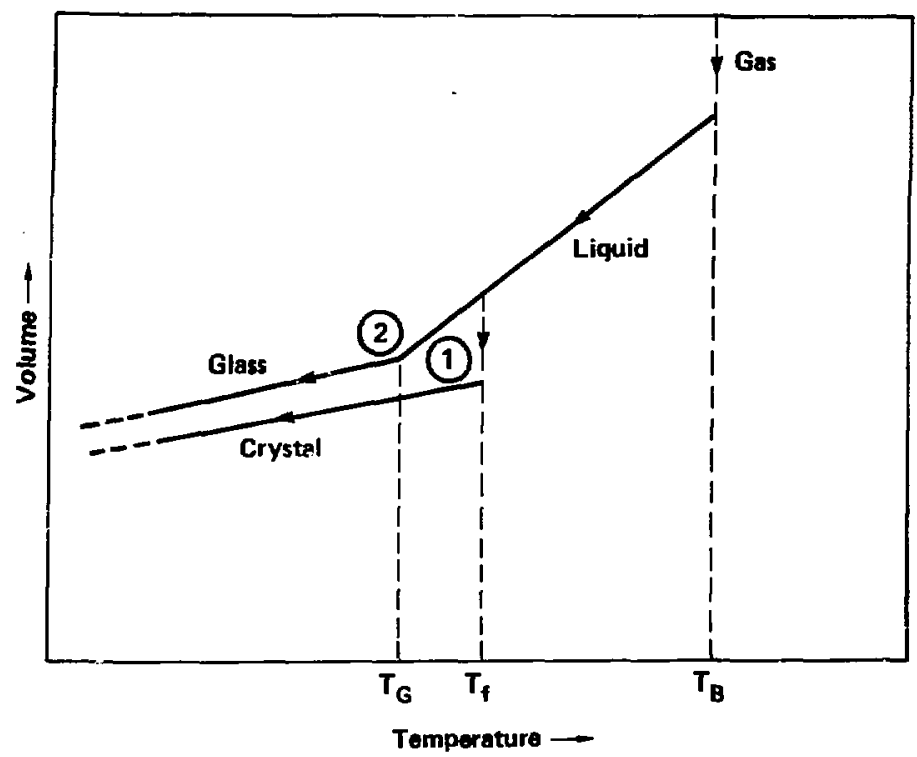

Fig. 2.1 Volume response to cooling of a hypothetical material.

are required to effect a density change of 100 percent. Intermolecular distances are now on the order of the molecular size and this tends to diminish the usefulness of methods used for calculating the EOS properties that were appropriate for densities far removed from the triple point. The finite density reflects the nature of the microscopic Intermolecular forces that repel at short distances $(\sim 4 A)$ and attract at large distances. The qualitative features of the intermolecular potential are similar to the volume dependence of the potential energy for the condensed phase which has a "stiff" short-range, repulsion and a longrange smoothly varying attractive portion. The attractive region of the potential supplies the cohesive properties of the fluid but the structure of the liquid is determined by geometric packing effects associated with 
its repulsive (virtually impenetrable) core. Though there is no longrange order (translational periodicity), the structure is constrained at short distances by the physics and chemistry of interatonic bond interactions and, therefore, possesses local order unlike the randomly distributed particles in the gas phase. The effect of the increasing density on molecular motion also distinguishes the liquid from the gas and solid phases. Atoms are becoming trapped by frequent nearest-neighbor collisions resulting in a combination of translation and oscillatory trajectories. The increased interaction also introduces a new component to the pressure and energy which is a unique property of the condensed state; the elastic component. The separation of molecules from one another requires work and this is due to the binding forces which differ in strength from the strongest covalently-bonded atoms (dissociation energies on the order of $100 \mathrm{Kcal} / \mathrm{mole}$ ) to the weaker van der Waals bonds (heats of sublimation on the order of $1 \mathrm{Kcal} / \mathrm{mol}$ ). These two components of the total pressure, therma i corresponding to the combined oscillatory and translational motion of atoms) and elastic, are both manifest in the condensed state and, as will be discussed in Chapter 3, are increased during shock compression.

As the liquid is cooled another transition will be observed; the liquid/solid transitios. In most cases (water being a notable exception), this transition is accompanied by a reduction in volume. The material's volume response to changes in temperature or pressure (compressibility) is again reduced, but only slightly, from the liquid state. At the microscopic level the atomic positions are locked in and, as a consequence, on the macroscopic scale, the material has an ability to 
resist shear stress. The transition to the solid state can proceed along either of two primary paths, the chofice depending largely on the cooling rate. With a relatively slow cooling rate, the atoms are allowed to position themselves in a configuration of minimum energy. This appears as a translationally periodic array, and is indicated as path 1 in Fig. 2.1. A discontinuous change in volume will accompany the transition. Detafls of the chemical bonding define the lattice structure. If the liquid is quenched rapidly (the rate depending on specific cases), the solid will form an amorphous (glass) structure where, as in the liquid phase, only local order exists. This is indicated by path 2 and displays a continusus change in volume around the glass transition temperature, $T_{G}$. Bond angles and lengths are preserved locally but long-range order is absent. Unlike the liquid state, however, the atoms keep their same nearest neighbors and, except on rare occasions, the notion is strictly vibrational about their equilibrium position. The viscosity of a material can be used to distinguish its liquid and solid state. On a scale most familiar to the reader, a material is generally considered solid if the application of a 20 pound shear force for 1 day to 1 cubic inch of material produces a permanent deformation of less than 0.007 inch. In terms of viscosity, this corresponds to about $4 \times 10^{14}$ poise, 16 orders of magnitude greater than the viscosity of water at room temperature.

The simple molecular motion and structural periodicity of the solid phase allow mathematical simplification of the complicated general vibrational problem to be reduced to a system of independent harmonic oscillators, each with their own vibrational frequency. The problem 
becomes one of solving for the frequency distribution function and predicts, with remarkable success, the simple properties of the solid for even the simplest forms of the distribution function. The theoretical treatments exercised on liquids are more involved than simply an extension of methods proven successful in treating the solid or gas phases. Extending the methods into the liquid phase tends to overemphasize properties which are characteristic to the phases specific to the model. It is better to start from first principles such as the equations of motion when discussing theoretical treatments of dense fluids.

\subsection{Theoretical Treatment of "Simple" Fluids}

According to classical mechanics, knowledge of the initial conditions of a collection of particles, namely their positions, velocities and the intermolecular forces, is sufficient to predict the state of the system at any later time. It is a fundamental problem of physics to predict the macroscopic state of a system from a knowledge of the microscopic interactions of the individual molecules. Molecular dynamics is an "exact" approach to the problem and illustrates the synthesis of thermodynamics from the interactions of the individual species. Trajectories are calculated for a respectable sample size $\left(\sim 10^{3}\right)$, given some initial conditions. After allowing for enough equilibrating collisions, the thermodynamic properties can be determined. Problems associated with a small sample size (such as boundary effects) can be minimized by using periodic boundary conditions. Results from computer experiments provide a standard by which the results of other theoretical methods of solution can be 
compared. Even with sophisticated computers, the anount of computation time required to solve a single problem warrants

an alternative approach, perhaps at the modest expense of being only a "good" approximation. A more parametric treatment is generally preferred when the state of the systems needs to be known for a large number of specified conditions such as different temperatures or densities, or when using different scaling parameters for the potential.

Perturbation theory is one approach which has been used to predict the thermodynamic properties of a large group of liquids, including the liquids of interest in this thesis, and compares favarably with dynamic EOS experimental results. The treatment is based on the hypothesis that the interatomic forces and energies are dominated by the sum of shortrange, pair interactions. This allows further sinplification by narrowing the dependance of all thermodynamic properties onto two functions: the pair potential, $u(r)$, and the pair distribution function, $g(r)$, which measures the probability of finding a particle at a given distance from a fixed particle in the fluid. Functional forms for $g(r)$ and $u(r)$ for a particular fluid can be calculated or obtained experimentally. One calculational method might involve choosing some model for the fluid [equivalent to choosing $u(r)$ ] and deriving forms for the pair distribution function either through molecular dynamics or by deriving suitable approximations for the higher order distribution functions in terms of the intermalecular potential and the pair distribution function. $x$-ray or neutron scattering experiments are commonly used to measure the structure factor, from which the pair distribution function can be determined. Ideally, the form of the potential can be evaluated by 
fitting the parameters of a suitable potential function to results of rigorous quantum mechanical calculations as Ree and Winter have done for the potential for two nitrogen molecules in various orientations. 50 Scattering experiments can also be used to measure the intermolecular potentia1. Argon-argon scattering experiments, for example, reveal the repulsive potential to be roughly exponential. Shock wave experiments can be used to infer the functional form of the potential and overlap the region of the potential sampled by molecular beam experiments. This is due to the high densities and temperatures involved in shock compression. The domination of influence on themodynamic properties by the short-range repulsive interaction is incorporated into the perturbation treatment and allows for a common grouping of liquids into distinct classes.

There are three basic types of molecular species which constitute the fluids investigated in this thesis. They are classified according to the character of the intermolecular forces which provides the cohesive properties of the fluid. The first class consists of spherical or nearly spherical molecules like argon and methane. The low boiling points of this class of liquids reflect the low cohesive energies involved with van der Wals bonding. They are also most amenable to rigorous theoretical calculations and form a group which are used to evaluate the successes of approximate methods. The next class, in the order of complexity, is the homopolar diatomics such as nitrogen and oxygen. These molecules are held together by strong covalent bond: $(\sim 200 \mathrm{kcal} / \mathrm{mole})$ and interact with other molecules through weaker van der Waals-type bonding i 5 Kcal/mole). Strictly speaking, the intermolecular potential for the diatomic molecules exhibit orientation dependence, at least under standard 
liquid conditions. The hydrocarbons represent the most complex class in this thesis. For these larger species, the internal vibrational contribution to the fluid's ability to store energy becomes significant. The relative ease of dissociating the larger units into a diverse collection of volatile subspecies (involving various $\mathrm{H}: \mathrm{C}$ ratios) requires additional considerations which are discussed in the following section. To a good approximation, the predissociated as well as the individual dissociated subspecies behave as though they are components of a "simple" fluid, at least with regard to the general features of the intermolecular potential. The term "simple" is meant to describe the intermolecular potential as having orientational independence with an attractive influence at large distances $(\geq 4 \lambda)$ due to dispersion forces, and repulsive influence at shorter distances $(\leq 4 \mathrm{R})$ due to electron overlap. The potential is actually an "effective" potential and includes many-body effects.

The analytical form for the pair-potential, chosen to represent a particular conformal class of fluids, is an important consideration. The criterion for a fluid to be conformal to some class is that the ratio $P_{C} V_{C} / R T_{C}$ equal approximately the same constant for any fluid in that class. Under shock-compressed conditions, however, the criterion becomes one of comparing the general features of the repulsive part of the potential. The similarities exhibited by a group (for example, $\mathrm{N}_{2}$ and $\mathrm{O}_{2}$ ) at high pressure and temperatures motivate the search for a functional form for that class which is spherically symetric with an energy scaling parameter multiplied by a universal function of the intermolecular separation. The rules of corresponding states are one way 
to scale the EOS in terms of variables reduced by their critical properties. The potential can be expressed as

$$
\Phi(r)=\varepsilon U\left(\frac{r}{r^{\star}}\right) .
$$

Three potentials used which are simple functions, satisfying the above criterion, and which represent good approximations for simple systens are the exponential-6, Lennard-Jones, and hard-sphere and are given by

$$
\begin{aligned}
& u(r)=\frac{c}{1-6 / \alpha}\left(\frac{\sigma}{a} \exp \alpha\left(1-\frac{r}{r^{\star}}\right)-\left(\frac{r^{\star}}{r}\right)^{6}\right), \\
& u(r)=4 \varepsilon\left(\left(\frac{\sigma}{r}\right)^{12}-\left(\frac{\sigma}{r}\right)^{6}\right), \\
& u(r)=\left\{\begin{array}{c}
+\infty, r<0 \\
0, r \geq 0 .
\end{array}\right.
\end{aligned}
$$

respectively. The exponential-6 (exp-6) form, in particular, is known from theoretical studies to be the correct functional form for molecular separations typical in a shock-compressed fluid. The scaling parameters, $\left(\varepsilon, r^{*}, \sigma\right)$, represent the well depth and collision diameters while a determines the degree of stiffness (Fig. 2.2). The EOS can be determired for a liquid, which obeys corresponding states scaling to a particular class, by choosing $u(r), g(r)$, along with an appropriate analytical procedure, and adjusting the scaling parameters with its critical point properties. Extensive coverage on the properties and theory of liquids can be found in Refs. 51-53.

The central approach to the perturbation method involves the expansion of the system of interest about some reference system. The thermodynamic properties of the system are then expressed in terms of the 


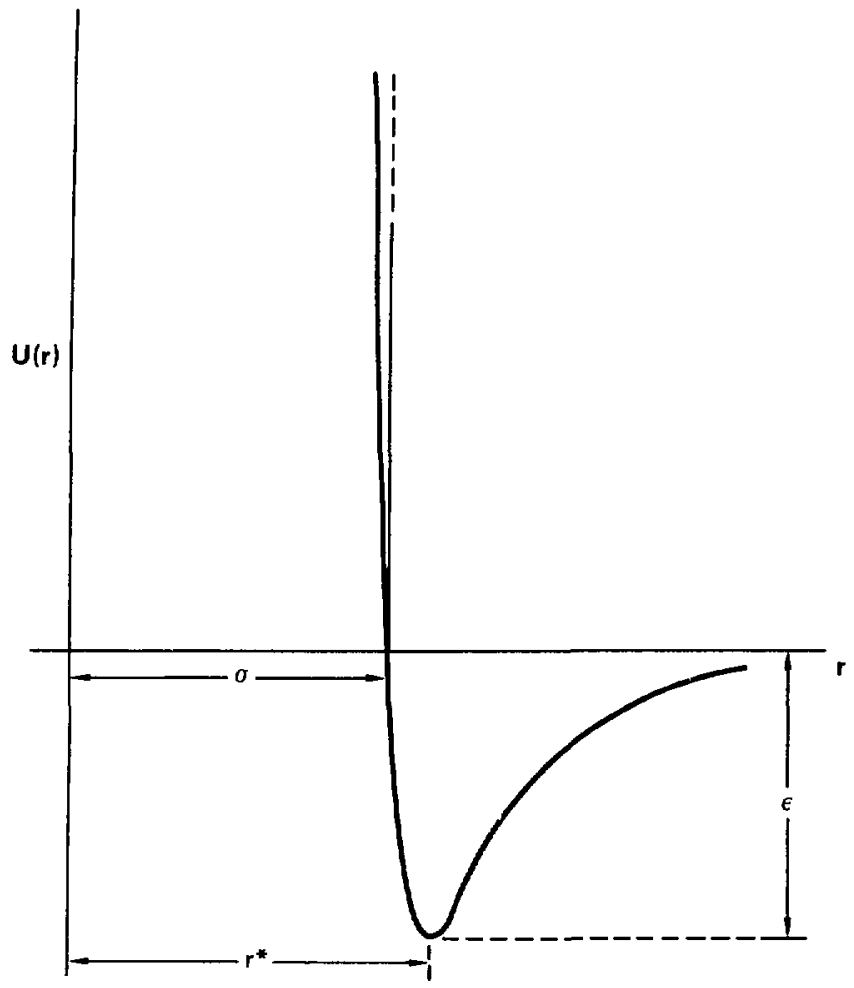

Fig. 2.2 Potentials frequently used in calculating properties of the liquid state.

known properties of the reference system. To lowest order the theory requires knowledge of the reference system free energy and pair distribution function. The potential is separated into the sum of two parts

$$
u(r)=u_{0}(r)+u_{1}(r)
$$

a stiff repulsive, reference potential, $u_{0}(r)$, and a weak perturbation, 
$u_{1}(r)$. The basic assumption is that the structure of the fluid is determined by hard-core effects. The use of the hard-sphere fluid becomes the obvious choice for the reference since information necessary for perturbation calculations is available in analytic form and is the simplest to treat. Agreement between molecular dynamics calculations, using the hard-sphere potential, and molecular beam experiments on argon to determine the radial distribution function indicates that a good fit can be obtained using a fairly simple form. Ross 54,55 utilized a variational approach to the perturbation method to calculate the EOS properties of liquid nitrogen and oxygen. In the "non-reactive" approach the interaction was assumed to be too weak to excite new vibrational or electronic levels in adjacent atoms. The partition function could be factored, thus allowing attention to be focused on the configurational part. The Helmholtz free energy of the system becomes the sum of the free energies of all degrees of freedom. Nothing unusual was involved in calculating the particular functions for free energies for the vibrational, electronic excitation, rotational or translational contributions. Improvements in the estimates for the free energy were made primarily on the configurational (interaction free energy) portion. Expansion of the Helmholtz free energy of the fluid to first order yields

$$
A_{\text {int }}<A_{H S}+F(n) H K T+\frac{N^{2}}{2 V} \int \phi(r) g_{H S}(r, n) d \vec{r},
$$

and is a rigorous upper bound on the free energy of the system. The variational parameter, $n=(r / 6)(N / V) d^{3}$, is the packing fraction and is adjusted to minimize the right hand side of Eq. (2.4); the better the choice for the reference system, the closer the expression can be brought to an equality. $A_{H S}$ is the Helmholtz free energy of the reference system 
(in this case the hard-sphere system), $d$ is the hard-sphere dianeter. is the number of particles in the system and $v$ is the volume. $g_{H S}(r, n)$ is the herd-sphere radial distribution function based on the Percus-Yevick equation. $F(n)$ is an empirical polynominal included so that Eq. (2.4) reproduces exactly the free energy for the inverse $12^{\text {th }}$ potential repulsive system. The pressure and energy are obtained from the free energy expression using thermodynamic identities such as

$$
\beta P / \rho=1+\rho\left(\frac{\partial \beta A}{\partial \rho}\right)_{T} \quad \text { and } \quad \beta E=\beta\left(\frac{\partial \beta A}{\partial \beta}\right)_{\rho} .
$$

where $\beta=1 / K T$.

The contribution from internal degrees of freedom includes rotational and vibrational, due to a number of independent oscillating modes, and electronic. The electronic partition function is an important contribution for oxygen in the temperature range of these experiments $(<1 \mathrm{eV})$ but is of little significance for nitrogen because of the high excitation energies. Ross' calculations accurately predict the results for nitrogen up to $30 \mathrm{GPa}$ and oxygen for the entire pressure range when compared with the dynamic EOS data (Figs. 1.1 and 1.2). A fluid's ability to follow corresponding states behavior reflects the validity in neglecting any deviations from pair-wise additivity and large scale electronic excitation or dissociation of the molecular structure. "Nonreactive" is meant to describe the molecular structure as remaining intact. As can be seen in Fig. 1.1, calculations for the "non-reactive" model tend to overestimate the pressure (also the temperature) for pressures above $30 \mathrm{GPa}$. This usually signals the onset of some increasingly important energy absorbing mechanism. Ross repeated the calculation and allowed for molecular dissociation. ${ }^{13}$ Agreement with 
experimental data is once again established. The dissociation energy for an isolated nitrogen molecule is about $9.7 \mathrm{eV}$, which is about an order of magnitude higher than the calculated temperatures accompanying a shock pressure of $60 \mathrm{GPa}$. The calculated fractional dissociation is on the order of 1 and Ross accounts for this with a density-dependent dissociation energy. As the molecules become more closely packed, the energy needed to break the triple bonds decreases. This can be illustrated by comparing the potentials between two nitrogen molecules and two nitrogen atoms. The equilibrium state realized by the fundamenta? constituents of the fluid, whether they be in the diatomic or monatomic form, is determined by two competing contributions to the total pressure: thermal and elastic. Suppose the form in Fig. 2.2a represents the intermolecular potential for two nitrogen molecules at standard conditions, $(P=1 \mathrm{~atm})$. Now consider another curve, identical to the $\mathrm{N}_{2}-\mathrm{N}_{2}$ potential, but translated along the $r$-axis such that the minimum, $r^{\star *}$, is just slightly to the left (smaller $r$ ) of $r^{\star}$. This curve represents the $\mathrm{N}-\mathrm{N}$ potential. The repulsive contribution to the energy is greater for the $\mathrm{N}_{2}-\mathrm{N}_{2}$ than for the $\mathrm{N}-\mathrm{N}$ interaction potential for any chosen density (or intermolecular separation, $r$ ) and so energetics favor the $N-N$ configuration. This is the elastic or "cold" contribution to the energy (or, equivalently, pressure). However, there is another consideration. The pressure is proportional to the number density of molecules, so in this instance energetics favor the diatomic state. From this argument, it seems reasonable that there exists a density or intermolecular separation where the two competing processes contribute equally to the pressure and that, for smaller separations, the monatomic form will be favored. Experimental results support this idea. 


\section{$2.3 \quad$ Mixtures}

Hydrocarbons are included in a group of liquids which form the next leve 1 of complexity. Ree developed methods to predict the thermodynamic properties of these liquids which agree with experimental data and which are based on the pair-wise additivity of spherically symetric "effective" potentials (as was done for the homopolar diatomics). 9,16,56 The added degree of difficulty depended on whether the original molecular structure was retained in the shock compression process. Ho new concepts are involved in the conformal solution theory for shock compressions too weak to dissociate the molecule. The system is treated with the same approach outlined in the previous section. Additional effort is involved to account for more complicated vibrational processes. Ree termed this predissociative approach the "non-reactive". model. For sufficiently high shock-pressures the larger molecular units will dissociate. A two-phase system, consisting of $\mathrm{C}-\mathrm{C}$ bonds in a diamond-like phase and $\mathrm{H}-\mathrm{H}$ bonds in a condensed molecular phase, has an energy that is lower than the initial C-H single-phase fluid. For intermediate degrees of shock compression other stoichiometric varieties may be present. Ree generalized the methods of the previous section to multicomponent systems or mixtures, and termed this the "reactive" model.

The "reactive" model considers a mixture of $N$ molecules of which $N_{i}$ are of species $i$ so that $N=\sum_{i} N_{i}$. These species are related according to a set of selected chemical reactlons involving various $H: C$ ratios

$$
v_{A} A+v_{B} B+v_{C} C+v_{D} D
$$


where the $v_{j}$ 's represent the stoichiometric coefficients and the capital letters represent the formulas of the compounds. The problem is to determine the equilibrium concentrations of the chosen species for a set of final thermodynamic states. The concentrations $\left[n_{k}\right]$ of the species are determined by minimizing the Gibbs free energy

$$
G\left(P, T,\left\{n_{k}\right\}\right)=\sum_{i} n_{i} p_{i}\left(P, T,\left\{n_{k}\right\}\right)
$$

which is the mixing function appropriate for fixed pressure, $P$, and temperature, $T$. The chemical potentials, $\mu_{j}$, are determined by the concentrations of the individual species through the use of an improved van der waals one-fluid model. In this model the properties of the mixture are taken to be those of a single hypothetical fluid or an ideal mixture of two or more such fluids. It is assumed that the radial distribution function has the same dependence for all the components. The exp-6 form of the potential is used and the scaling properties are determined by

$$
\begin{aligned}
& r^{\star}=\sum_{i j} x_{i} x_{j}\left(r_{j j}^{*}\right)^{3}, \\
& \varepsilon^{\star}=\sum_{i j} \varepsilon_{j} x_{i} x_{j}\left(\frac{r_{i j}^{*}}{r^{\star}}\right)^{3}, \\
& a=\sum x_{i} x_{j} \alpha_{i j} \frac{\varepsilon_{i j}}{\varepsilon^{\star}}\left(\frac{r_{i j}}{r^{\star}}\right)^{3},
\end{aligned}
$$

where $x_{i}=\frac{n_{i}}{\sum n_{j}}$ is the mole fraction of species $i$ in the fluid or solid phase. The ${ }^{j}$ scaling parameters for each of the species are adjusted according to their critical point and argon values through

$$
\frac{\varepsilon}{\varepsilon_{A}}=\frac{T_{C}}{T_{A C}} \quad \cdot \frac{r^{*}}{r_{A}^{*}}=\left(\frac{V_{C}}{V_{A C}}\right)^{1 / 3}
$$


and by the Lorentz-Berthelot rule,

$$
c_{i j}=\sqrt{\varepsilon_{i j} \varepsilon_{j j}}, \sigma_{i j}=\frac{1}{2}\left(\sigma_{i j}+\sigma_{j j}\right),
$$

with an additional rule for $\alpha_{i j}$ given by

$$
\alpha_{i j}=\sqrt{\alpha_{i j} \alpha_{j j}}
$$

The EOS properties and stoichiometric concentrations are determined by the following sequence of calculations which are contained in a computer code CHEO developed by F. H. Ree. ${ }^{3}$ Initial concentrations are chosen for each of the species. These are used in determining the scaling parameters which become incorporated into the expression for the Helmholtz free energy ( $\mathrm{Eq} .(2.5)$ ). The free energy is minimized with respect to the hard-sphere dianeter and depends implicitly on reactant and product concentrations, $n_{i}$. The chemical potential for the solid carbon phase is given by

$$
\mu_{i}=\mu_{i}^{0}(T)+\int_{1 \text { atm }}^{P} V_{i}(P) d P
$$

The pressure, P, and total energy, E, are calculated using the interaction free energy, $A_{\text {int }}$, and total free energy, $A$ (which is the sum of free energies of all degreas of freedom),

$$
\begin{aligned}
& \frac{\beta P V}{N}=1-\left(\beta \frac{\partial A_{i n t}}{\partial V}\right)_{T}, \\
& \frac{\beta E}{N}=\left(\frac{3}{2}+\beta \frac{\partial(\beta A / N)}{\partial \beta}\right)_{V}+\beta E_{R V E},
\end{aligned}
$$

where $E_{R V E}$ is the energy from the internal degrees of freedom. The Grineisen form for the: molar volume is used in Eq. (2.13) and relates the values of $P, E$ and $V$ to iheir values of $0 K .^{16}$ values for the chemical 
potentiais in the standard reference state, $\mu_{i}^{0}(T)$, are from constantpressure specific heats, enthalpy of formation, $\Delta H^{\circ}$, and entropies, $S^{*}$. The procedure will be discussed in Chapter 6 . The chenical potentials are used in the expression for Gibbs free energy (Eq. (2.7)) which is minimized with respect to the concentrations of the species subject to chemical balance. This completes one iteration. The procedure is repeated until a convergence criterion is satisfied for each chosen ( $T$, P) state. A typical problem involves choosing the hydrocarbon species, defining a set of chemical reactions (Eq. (2.6)), and solving the thermodynamic variables $\left(E, P, U_{S}, U_{p}\right)$ for given $(T, P)$. The Huganiot represents the final thermodynamic state of interest and the number of degrees of freedom on the EOS surface can be further reduced by forcing them to satisfy a Hugoniot relation (Chapter 3 ). Figure 1.3 compares theoretical results with experimental data for shock compressed benzene. The "non-reactive" model shows good agreement with the data below 13 GPa. Above $13 \mathrm{GPa}$, where dissociation becomes significant, the "reactive" model agrees. 


\section{CHAPTER 3. SHOCK WAVES}

\subsection{Introduction}

Most materials support shock waves because the sound velocity increases with pressure. Only planar shocks are considered here. Uniaxial strain can be achieved by uniformly loading the surface of a semi-infinite sample; for example, by using a piston. Each element of fluid pushes the neighboring fluid out of its way and thereby propagates a pressure pulse into the fluid. A steady state will be arrived through a series of transient flows which asymptotically approach the steady-state condition after a time which depends on the shock thickness and propagation velocity. A convenient examination of a steady-state condition can be achieved by placing a coordinate systen stationary with re spect to the particle velocity behind the shock front. The kinetic energy of the directed molecular motion is converted to kinetic energy of random motion (heat) when successive fluid elements collide. This irreversible heating which accompanies the compression process is useful. for studying activation-type processes such as molecular dissociation and electronic excitation. The following section will introduce shock wave nomenclature and concepts through the use of a simple model proposed by Band and Duvall. ${ }^{57}$ Section 3.3 will examine shock wave formation. This is best illustrated from a continuum mechanics approach. The last three sections will cover the Hugoniot relations, shock compression of distended materials and impedance matching. 


\section{$3.2 \quad 1-0$ Elastic Brad Mode1}

The elastic bead model is an examination on an atomistic level of the propagation of a disturbance initiated by a piston moving to the right at velocity, $v_{0}$. The medium, in this $1-d$ problem (Fig. 3.1), consists of a string of perfectly elastic beads of diameter, $\sigma$, that are initially separated a distance, $s$, and allowed to slide on a frictionless wire. At $t=0$, the first bead is struck by the piston. Conservation of momentum and energy dictates that the bead will move to
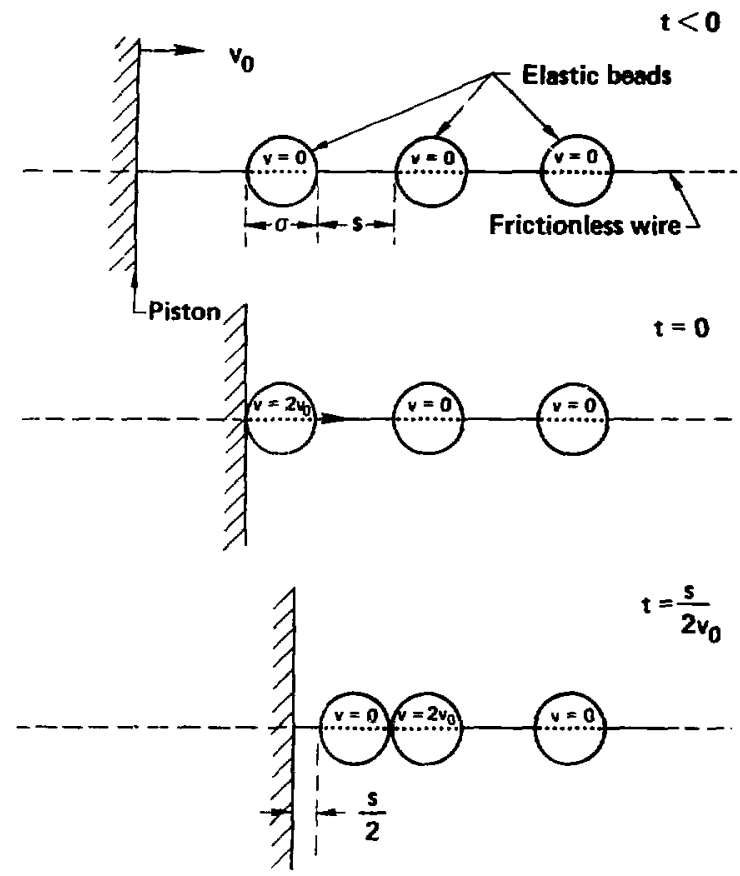

Fig. 3.1 Bead model used to illustrate basic shock wave concepts. 
the right at velocity $2 v_{0}$ since the piston is assuned to be infinitely massive. At the time of collision with the next bead $\left(t=\frac{s}{2 v_{0}}\right)$, the first bead will exchange its momentum with the second bead, leaving the first bead with velocity $v=0$. This disturbance covers a distance $n(s+\sigma)$ in a time $n \frac{s}{2 v_{0}}$, where $n$ is the number of beads experiencing a collision. Therefore the front moves at velocity

$$
u_{s}=2 v_{0}\left(\frac{s+g}{s}\right)
$$

which is identified as the stock velocity. The velocity history of the first bead appears in Fig. 3.2. At $t=\frac{2 s}{2 v_{0}}$ the piston reaches the first bead and the process continues. The average velocity of any bead turns out to be the piston velocity, $v_{0}$, which is identified as the particle velocity, $u_{p}$. Consequently, the $u_{p}-u_{s}$ relation for this model is linear. The motion of all the beads can be represented on an $x-t$ diagram (Fig. 3.3). There it can be seen that the average particle velocity is equal to the piston velocity and that the shock front travels faster than the mean particle velocity.

The mean kinetic energy associated with the average bead velocity, denoted by $k$, is

$$
K=\frac{1}{2} m u_{p}^{2}
$$

The total kinetic energy of each bead is

$$
H=\frac{1}{2}\left\{\frac{1}{2} m\left(2 u_{p}\right)^{2}+\frac{1}{2} m(0)^{2}\right\}=m u_{p}^{2}
$$




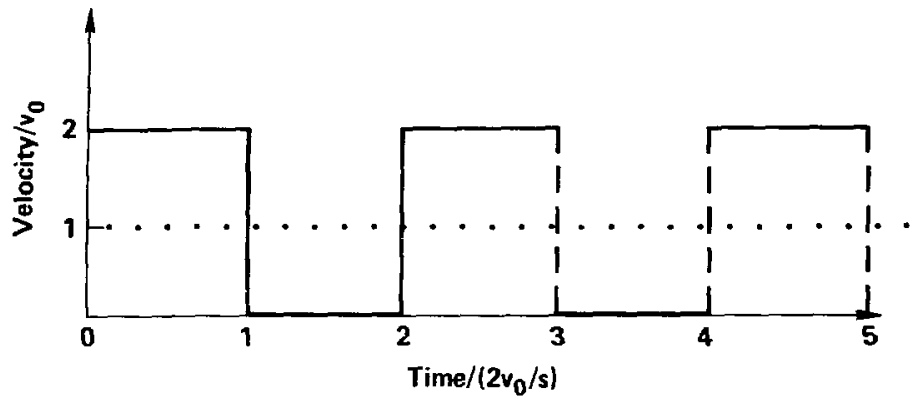

Fig. 3.2 Velocity history of the first bead.

where the first term in the bracket represents the kinetic energy wile the bead is moving (half the time) and the second while the bead is stationary. The total kinetic energy exceeds the average kinetic energy that is assigned to the average particle velocity and represents an excess energy corresponding to thermal agitation which is analogous to

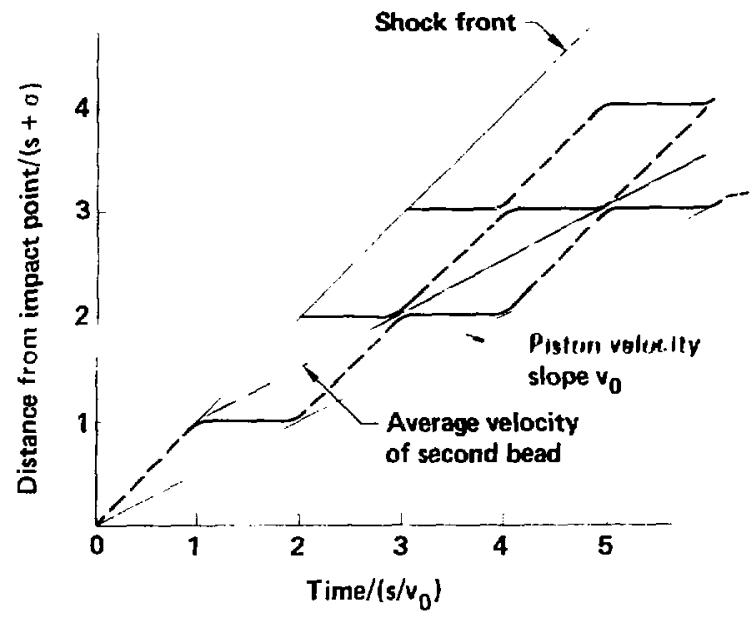

Fig. $3.3 \times-t$ diagram showing the trajectory of each bead. 
internal energy. Denoting this excess by $E$, it follows,

$$
E=\frac{1}{2} m u_{p}^{2}
$$

The rate of energy increase is equal to the total energy acquired by each bead $\left(\Delta H=m U_{p}^{2}\right.$ ) per time interva) $\left(\frac{s}{2 v_{0}}\right)$ and equals the average force executed by the piston per unit area (P) times the velocicy, $v_{0}$. Therefore the pressure becomes

$$
P=\frac{2 m}{s} u_{p}^{2}
$$

The average density of the undisturbed region, $\rho_{0^{*}}$ is the number of beads per unit length

$$
\rho_{0}=\frac{m}{s+o} \text {. }
$$

The density of the disturbed region can be found by taking the ratio of the number of beads set into motion at some time, $t$, to the length of the disturbed region which is bounded by the shock front on the right, $x_{F}=\frac{2 v_{0} t}{s}(s+a)$, and the piston, $x_{p}=v_{0} t$, on the left. This gives the final density

$$
\rho=\frac{2 m}{2 \sigma+s} \text {. }
$$

The ratio of the final to initial density is

$$
\frac{e}{\rho_{0}}=\frac{2 \sigma+2 s}{2 \sigma+s}
$$

which, for this model, is independent of the shock pressure. The relation between the pressure, particle, and shock velocity (Eq. 3.5) is consistent with the Hugoniot relation for the pressure (Section 3.4), which the reader may verify. The $P-V$ states $(V=1 / \rho)$ attainable in a $1-d$ 
shock transition for an infinitely stiff potential lie on a vertical line (fig. 3.4). The final state for real fluids exhibits a more gradual approach to a limiting volume but behaves in a qualitatively similar manner.

\subsection{Shock Wave Formation}

The formation of a shock wave is a consequence of two campeting processes: The wave tends to steepen due to the higher sound velocity in the shocked material and the wave tends to spread out due to the increasing influence of dispersion and dissipative effects. Microscopic fluid transport theory can be used to give a detailed description of the processes which take place in a shock compression processes. This

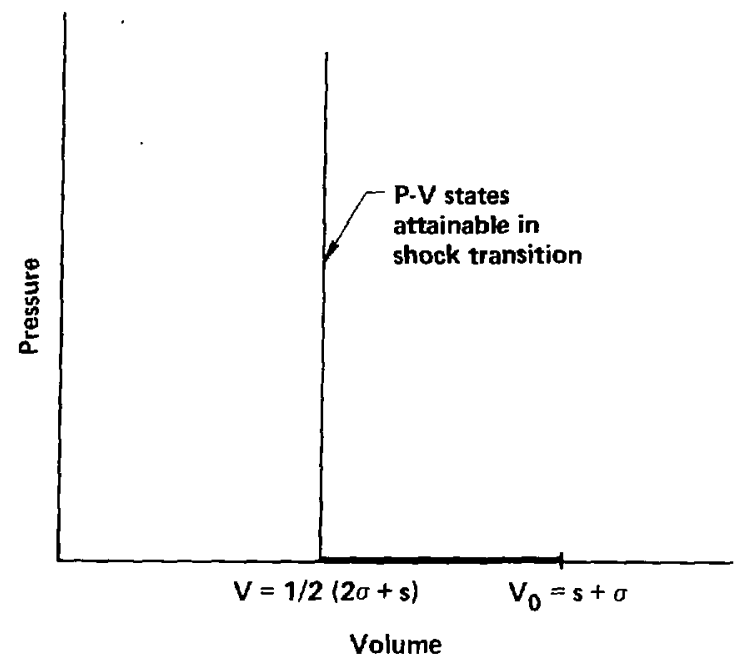

Fig. 3.4 Hugoniot in P-V space for the bead model. 
involves following the trajectories of individual interacting particles. The course to be pursued, though less precise, relies on the validity of assuming local equilibrium between macroscopic fluid elements. Constitutive models, which relate flows to gradients, are an important feature to a continuum approach. As it turns out, the macroscopic treatment agrees quite well with the microscopic treatment, considering the large gradients involved, and also serves to illustrate important mechanisms responsible for the final shock profile. ${ }^{58}$

The conservation relations can be derived from first principles with no reference made to a specific material. A differential treatment relies on the continuity in space and time of local densities, energies and particle velocities. Mass conservation states that the amount of material which accumulates in some volume is equal to the total rate of flow through the boundary surface and in differential form is

$$
\frac{\partial}{\partial t} \rho+\nabla(\rho \bar{v})=0 \quad \text {, }
$$

where $\rho=\rho(\bar{x}, t)$ is the mass density of the system at the point $\bar{x}$ at time $t$ and $\bar{v}=\bar{v}(\bar{x}, t)$ is the mass velocity. ${ }^{51}$ similarly, the rate of change of momentum in a volume is equal to the total force acting on the mass within the volume in addition to the rate at which momentum follows in and out of the volume. The forces can be external, such as gravitational, or can be forces exerted by the fluid surrounding the volume. The momentum conservation equation is

$$
\frac{\partial}{\partial t}(\rho \bar{v})+\nabla \cdot(\rho \bar{v} \bar{v})=\rho \bar{F}-\nabla \cdot \underset{\approx}{p} .
$$

The second term on the left hand side of the momentum equation represents 
the momentum flowing across the surface. The first term on the right hand side represents the external forces $(\bar{F}=$ force per unit mass) acting on the mass inside the volume and the second term is the total pressure force acting upon the mass within the volume, where $\underset{\approx}{\mathrm{p}}$ is the pressure tensor. The energy conservation equation is derived by equating the rate of change of energy within a volume to the combined effects of the work done on the mass within the volume, the convective energy flow outside of the volume, and conductive energy flow from the volume. The tatal energy, $E$, includes the potential energy due to the external forces, $E^{\prime}$, and the kinetic energy due to mass flow $\left(1 / 2 \rho u_{p}^{2}\right)$. The expression for energy conservation becomes

$$
\rho \frac{\mathrm{DE}}{\mathrm{Dt}}=-\underset{\approx}{\mathrm{P}}: \bar{\nabla} v-\bar{\nabla}+\bar{q}
$$

where $\bar{q}$ is the heat flux vector and $\frac{D}{D t}$ is the convective derivative, $\frac{D}{D t} \equiv \frac{\partial}{\partial t}+\bar{v} \cdot \nabla$.

Up to this point only the notion of a continuum has been invoked. To go further in predicting the flows, constitutive models of the material are needed, which relate flows to gradients. These empirical relations are another approximation in the continuum approach. Fourier's law relates the heat flux vector, $\bar{q}$, to the gradient of the temperature, $T$, by a proportionality, $k$, called the thermal conductivity coefficient and is a material property;

$$
\vec{q}=-\kappa \vec{\nabla} T
$$

The pressure tensor consists of contributions from the equilibrium scalar component, $P$, and the shear and bulk viscosity components which are 
proportional to gradients of the particle velocity,

$$
\underset{\approx}{p}=\left[P+\left(\frac{2}{3} n-n_{v}\right) \bar{\nabla} \cdot \bar{v}\right] \underset{\approx}{1}-2 \eta \operatorname{sym}(\nabla \bar{v}) .
$$

In Eq. (3.13), $n$ is the coefficient of shear viscosity which is a measure of the resistance to shear force, $n_{v}$ is the coefficient of bulk viscosity which is a measure of the resistance to compressive forces, and

$$
\operatorname{sym}(\nabla \bar{v})_{i j} \equiv \frac{1}{2}\left(\frac{\partial \bar{v}_{i}}{\partial x_{j}}+\frac{\partial \bar{v}_{j}}{\partial x_{j}}\right) \text {. }
$$

By incorporating the constitutive relations into the conservation equations and with the aid of the second law of thermodynamics TdS $=d E+$ PdV, where $S$ is the entropy per unit mass, an expression for the Navier-Stokes equation is obtained,

$$
\begin{gathered}
\frac{\partial \bar{v}}{\partial t}+(\bar{v} \cdot \nabla) \bar{v}=\bar{F}-\frac{\nabla \rho}{\rho}+\frac{\eta}{\rho} \nabla^{2} \bar{v}+\frac{1}{\rho}\left(\eta_{V}+\frac{1}{3} n\right) \nabla \cdot(\nabla \cdot \bar{v}) \underset{\approx}{I}, \\
\frac{\partial \rho}{\partial t}+\nabla(\rho \bar{v})=0, \\
\frac{\partial S}{\partial t}+(\bar{v} \cdot \bar{\nabla} S)=\frac{K}{\rho T} \nabla T+\frac{\eta}{2 \rho T}\left(\frac{\partial v_{j}}{\partial X_{K}}+\frac{\partial v_{K}}{\partial X_{i}}-\frac{2}{3} \delta_{i j} \nabla \bar{v}\right)^{2}+\frac{\eta}{\rho T}(\nabla \cdot \bar{v})^{2} \\
d \rho=\left(\frac{\partial \rho}{\partial P}\right)_{S} d P+\left(\frac{\partial \rho}{\partial S}\right)_{P} d S
\end{gathered}
$$

which is a set of hydrodynamic equations governing the flow of a viscous fluid. The first term on the right hand side of the entropy equation corresponds to heating or cooling of the fluid by heat conduction and, as a whole, tends to increase the entropy. The second and third terms represent the mechanical energy dissipated by viscosity per unit volume per unit time and are also responsible for increasing the entropy. The relative importance of the viscous terms for the conditions appropriate 
in shock compression can be demanstrated by equating the inertial forces in the equation of motion (first equation of 3.15 ) to the viscous forces. Letting $U_{p}$ represent the scale of the flow velocity and $\delta$ the characteristic dimension of the flow region, an estimate of the relative importance of the inertial term (left hand side of the momentum equation) can be made and is of the order $\rho v_{p}{ }^{2} / \delta$. The viscous term is of the order $n U_{p} / \delta^{2}$ and the ratio forms the reciprocal Reynolds number

$$
\frac{\text { viscous }}{\text { inertial }} \sim \frac{1}{R_{e}} \sim \frac{\eta}{\rho U_{p} \delta} \text {. }
$$

Similarly, comparing heàt transfer by conduction in the energy equation, which is of the order $\times \Delta T / \delta^{2}$, to the mechanical energy transfer, which is of the order $\rho \frac{\mathrm{p}}{\delta} \Delta E$, the reciprocal peclet number can be formed

$$
\frac{\text { thermal }}{\text { mechanical }} \sim \frac{1}{\mathrm{P}_{\mathrm{e}}} \sim \frac{k}{\rho U_{\mathrm{p}} \delta C_{\mathrm{p}}} \text {, }
$$

where the definition of specific heat at constant pressure, $C_{p}=\left(\frac{\Delta H}{\Delta T}\right)_{p}$, has been included. Viscous and heat conduction terms become important when the Reynolds and Peclet numbers are on the order of unity. An estimate of the values will appear at the end of this section.

When the dissipative terms in Eq. (3.15) are neglected, the Euler equations result;

$$
\begin{aligned}
& \frac{\partial \bar{v}}{\partial t}+(\bar{v} \cdot \nabla) \bar{v}=\frac{-c^{2}}{\rho} \bar{v}_{\rho}, \\
& \frac{\partial \rho}{\partial t}+\nabla(\rho \bar{v})=0,
\end{aligned}
$$

with the sound velocity defined as

$$
c^{2}(\rho)=\left(\frac{\partial P(\rho, S)}{\partial \rho}\right) S=\text { const }
$$


If it is assumed $p=p(v)$ and $c=c(v)$, then the equations describe a simple wave in terms of, for example, the particle velocity

$$
\frac{\partial v}{\partial t}+[v+c(v)] \frac{\partial v}{\partial x}=0 .
$$

The ideal gas relation for the adiabat

$$
P=P_{0}\left(\frac{e}{p_{0}}\right)^{r}
$$

produces the sound veloctty

$$
c^{2}=c_{0}^{2}\left(\frac{e}{\rho_{0}}\right)^{\gamma-1}, c_{0}=c\left(p_{0}\right) .
$$

The coefficient $r$ is the ratio of specific heats, $C_{p} / C_{v}$. Finally, the genera! solution of Eq. (3.19) has the form

$$
v=f(x \pm g(v), t), g(v)=c_{0}+\frac{x+7}{2} v,
$$

where $f(x)$ is an arbitrary function defining the wave shape at $t=0$. The solution, for a sound velocity independent of flow velocity, describes the propagation of a wave with constant shape. However, the scund velocity, $g(v)$, increases with $v$ since the adiabat is concave upward and results in a steepening of the wave profile in regions of higher flow velocity, $v$ (Fig. 3.5). If this were allowed to occur indefinitely, the flow veloctty, $v(x)$, would become multi-valued, which has no physical meaning. Before this could occur the flow gradients would become too large to justify neglect of the dissipative terms in the equation.

Inclusion of dissipative terms complicates the fundamental equation with regard to a solution in closed analytical form unless only second-order, non-linear terms are considered and it is assumed that 


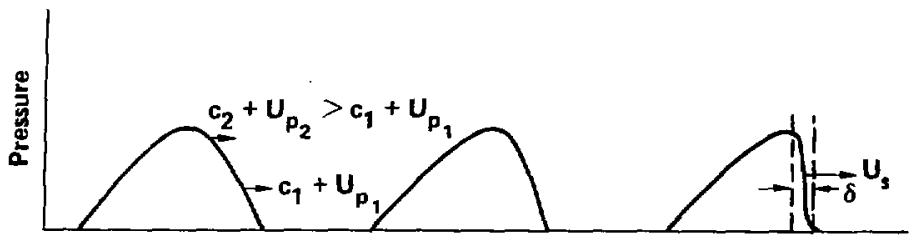

Propagation distance

Fig. 3.5 Shock wave formation.

dissipation coefficients are small to first order. Correspondingly, the linear dissipative terms will be small to second order and the non-linear dissipative terms may be neglected. To this degree of accuracy and assuming propagation along the $x$-axis, the equations become

$$
\begin{aligned}
\rho\left(\frac{\partial v}{\partial t}+v \frac{\partial v}{\partial x}\right) & =-\frac{\partial P}{\partial x}+\left(\frac{4}{3} \eta+\eta_{v}\right) \frac{\partial^{2} v}{\partial x^{2}}, \\
\frac{\partial S}{\partial t}+v \frac{\partial S}{\partial x}=\frac{\kappa}{\rho T} \frac{\partial^{2} T}{\partial x^{2}}, & \\
\frac{\partial P}{\partial t}+\frac{\partial P}{\partial x} v+c^{2} \rho \frac{\partial v}{\partial x} & =-\frac{c^{2} k}{\rho T}\left(\frac{\partial \rho}{\partial S}\right)_{P} \frac{\partial^{2} T}{\partial x^{2}} \\
& \left.\left.=-\frac{c^{2} k}{\rho T} \frac{\partial \rho}{\partial S}\right)_{P} \frac{\partial T}{\partial P}\right)_{S} \frac{\partial^{2} P}{\partial x^{2}}
\end{aligned}
$$

These equations constitute a complete system of equations for unidimensional flaw correct to second order in the density perturbation. $^{59}$ With additional assumptions and simplifications regarding the forms chosen for the pressure and density dependence, the Burgers equation for a quasi-simple wave follows

$$
\frac{\partial u}{\partial \tau}+u \frac{\partial u}{\partial \xi}=n_{L} \frac{\partial^{2} u}{\partial \xi^{2}}
$$


where

$$
\begin{aligned}
& E=x-c_{0} t, \quad \tau=t, u=\frac{x+1}{2} v, \\
& \text { and } n_{L}=\frac{1}{2 p_{0}}\left[\frac{4}{3} n+n_{v}+\kappa\left(\frac{1}{C_{v}}-\frac{1}{C_{p}}\right)\right] .
\end{aligned}
$$

Suppose the initial perturbation is bounded but otherwise arbitrary such that

$$
\int_{-\infty}^{\infty} U_{0}(\xi) d \xi=H<\infty
$$

Then, for large $\tau$, the asumptotic form of the solution becomes

$$
u(\xi, \tau) \rightarrow-2 n_{L} \frac{d}{d \xi} \ln F\left(\frac{\xi}{\sqrt{4 n_{L} \tau}}\right) \text {, }
$$

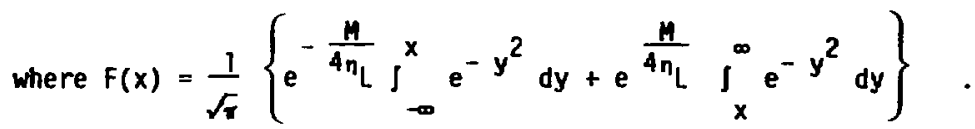

This solution of the Burgers equation also has a stationary solution describing a wave moving without deformation at constant velocity, w;

$$
u=f(\xi \pm W \tau)
$$

where

$$
\begin{aligned}
f(\xi) & =U_{0}+\frac{\Delta u}{1+\exp \left(\frac{\Delta u x}{2 n_{L}}\right)} . \\
w & =U_{0}+\frac{\Delta u}{2} .
\end{aligned}
$$

and $U_{0}$ and $a$ are integration constants. This solution represents a shock wave with a jump, su, in the flow velocity and a transition width

$$
\delta=\frac{2 n_{L}}{\Delta u} .
$$


An estimate for the shock width can be made for the strongest shock reported in these experiments for nitrogen at $P=0.600$ mbar, $P=2.7$ $\mathrm{g} / \mathrm{cm}^{3}, U_{p} \simeq 7 \times 10^{5} \mathrm{~cm} / \mathrm{s} . \quad T=12000 \mathrm{~K}$. Consider the transverse momentum change between adjacent layers separation by a characteristic intermolecular distance, $\sigma$. If the transfer occurs in a time wich scales with the vibrational frequency of the molecules, $v$, such that

$$
v \sim \frac{v_{\mathrm{TH}}}{\sigma} \sim \frac{\sqrt{3 \mathrm{KT} / \mathrm{m}}}{\sigma} \sim 10^{13} \mathrm{sec}^{-1}
$$

then the rate of momentum transfer per unit cross-section, $\sigma^{2}$, is of the order

$$
\frac{m \frac{\Delta u_{x}}{\Delta y} \sigma v}{\sigma} \sim n \frac{\Delta u_{x}}{\Delta y} \Rightarrow n \sim \frac{m v}{\sigma} .
$$

This gives a viscosity of $2 \times 10^{-2}$ poise and a corresponding shock thickness, $\delta$, of $2 \AA$, which is well within an order of magnitude accuracy when compared with the exact resuits for argon at similar conditions. 58 This estimate for the shock thickness actually represents a reaction zone corresponding to a translational relaxation phenomena involving momentum exchange through collisions. This is sufficient for simple molecules like argon. Nitrogen, however, has several modes of relaxation which reguire exchange through a hierarchy of the various degrees of freedom. The relaxation time for energy transfer from translational to vibrational is on the order of $5 \times 10^{-10}$ seconds which gives a shock width of approximately 5 microns. 60

The Reynolds and Peclet number can also be estimated to the same order of accuracy if it is assumed 


$$
\begin{aligned}
& \kappa \sim C_{v} U_{p} \sigma \sim .01 \text { watts } \mathrm{cm}^{-1} \cdot \mathrm{K}^{-1}, \\
& \text { where } C_{v} \sim \frac{\Delta E}{T} \sim 1 \text { joules } \cdot \mathrm{cm}^{-3} \mathrm{~K}^{-1} .
\end{aligned}
$$

In this case

$$
\frac{1}{P_{e}} \sim \frac{\text { themal }}{\text { mechanica })} \sim \frac{k}{U_{p} \delta C_{v}} \sim \frac{10^{-2}}{\left(10^{6}\right)\left(10^{-8}\right)(1)} \sim 1
$$

and $\frac{1}{R_{e}} \sim \frac{\text { viscous }}{\text { inertial }} \sim \frac{n}{U_{p} \delta} \sim \frac{10^{-2}}{\left(10^{6}\right)\left(10^{-8}\right)} \sim 1$.

These order-of-magnitude estinates demonstrate the importance of including dissipation when dealing with moderate strength shocks $(\sim 1$ Mbar). It is impossible to construct a continuous distribution of all wave parameters for a sufficiently strong wave without including viscosity.

In this section, the intent was to show that some interesting. quantitative processes in shock wave physics are tractable, with minor approximations, using a conbination of first-principle conservation relations and material property-dependent constitutive models. It has been shown that the formation and structure of a shock wave requires both dissipative effects and the non-linear nature of the sound velocity dependence on the flow field parameters. The flow equations will also be needed in the next section. Instead of incorporating constitutive models and appealing to arguments as to what terms can be neglected, a principle will be invoked which was briefly mentioned: Relaxation times are negligible compared to the duration of an experiment. 


\section{$3.4 \quad$ Hugoniot Relations}

Recall that there exist certain relations between the inftial and final thermodynamic flow variables in the simple bead model of Section 3.1 which related the two equilibrium states. Similar relations may be derived for a general material with no reference made to a specific model since appeals are made only to conservation of mass, monentum and energy. The only stipulation is that far enough away from the shock transition region, which was shown to be on the order of a few microns, the state of the fluid is in themodynamic equilibrium.

The process is steady and uniaxial. Partial derivatives with respect to time vanish and partial derivatives with respect to the spatial coordinate, $x$, can be replaced by total derivatives leading to the conservation equations

$$
\begin{aligned}
& \frac{d}{d x}(\rho v)=0, \\
& \frac{d}{d x}\left(P+\rho v^{2} \quad n_{L} \quad \frac{d v}{d x}\right)=0, \\
& \frac{d}{d x}\left[\rho v\left(H+\frac{v^{2}}{2}\right) \quad n_{L} v \frac{d v}{d x}-\propto \frac{d T}{d x}\right]=0 .
\end{aligned}
$$

The shear and bulk viscosity coefficients have been combined into a single term, $n_{L}$, defined by the expression following Eq. (3.24) and is called the longitudinal viscosity coefficient. Equilibrium times for the first coefficient are on the order of the translational collision time. If the time required for establishing the static pressure is of the order of the mean-free-path, then $\eta_{v}$ is on the order of $n$ and, in the uniaxial case, the combination can be justified. ${ }^{61}$ There has also been a 
change in variables, using the second law of thermodynamics, TdS $=\mathrm{dH}-$ VdP.

If it is assumed that the shock transition region is an infinitely thin layer, which is equivalent to ignoring the viscosity and heat conduction terms, and that the flow parameters in the undisturbed and shocked region are denoted by subscripts " 0 " and " 1, "respectively, then it follows that the first integral of Eqs. (3.30) gives

$$
\begin{gathered}
\rho_{1} v_{1}=\rho_{0} v_{0}, \\
p_{1}+\rho_{1} v_{1}^{2}=p_{0}+\rho_{0} v_{0}^{2}, \\
\rho_{1} v_{1}\left(H_{1}+\frac{v_{1}^{2}}{2}\right)=\rho_{0} v_{0}\left(H_{0}+\frac{v_{0}^{2}}{2}\right) .
\end{gathered}
$$

These conservation equations relate the flow and thermodynamic variables across the discontinuity in an arbitrary reference system. The unperturbed state, denoted by subscript " $0, "$ is chosen as stationary and the reference frame is fixed with respect to the shock front. Subscripts on the variables corresponding to the shocked state are removed thus obtaining the Hugoniot relations,

$$
\begin{aligned}
\rho_{0} & =\rho\left(1-\frac{U_{\mathrm{D}}}{U_{s}}\right), \\
P-P_{0} & =\rho_{0} U_{S} U_{D}, \\
H_{1}-H_{0} & =\frac{1}{2}\left(P-P_{0}\right)\left(\frac{1}{\rho_{0}}-\frac{1}{\rho}\right),
\end{aligned}
$$

where $U_{p}$ represents the particle velocity in the shock compressed region. The energy equation can be expressed in terms of an internal 
energy difference by substituting $H=E+P V$ :

$$
E-E_{0}=\frac{1}{2}\left(P-P_{0}\right)\left(V_{0}-V\right) \text {, }
$$

where $v=\frac{1}{\rho}$

The first two Hugoniot equations involve shock parameters, such as particle and shock velocity, in addition to thermodynamic variables while the third equation relates strictly thermodynamic quantities. The relation must be satisfied if the shock exists and since the end states are in thermodynamic equilibrium, they lie on the equilibrium surface. The locus of P-V states satisfied by the Hugoniot relation on the equilibrium surface is called the Hugoniot. The location of the Hugoniot in the P-V plane with respect to the isentrope can be determined by considering two identical specimens with the same initial state $\left(P_{0}, V_{0}\right)$; one shock compressed to pressure $P_{1}$ and the other to $P_{1}+d P_{1}$. Equation (3.37) can be differentiated in order to determine the difference in specific external energy

$$
d E_{1}=\frac{1}{2}\left(V_{0}-V_{1}\right) d P_{1}-\frac{1}{2}\left(P_{1}+P_{0}\right) d V_{1}
$$

Combining this expression with the second law of thermodynamics yields

$$
T_{1} d S_{1}=d E_{1}+P_{1} d V_{1}=\frac{1}{2}\left(V_{0}-V_{1}\right)\left[1-\left(\frac{U_{S}-U_{p}}{C_{1}}\right)^{2}\right] d P_{1} .
$$

where Eq. (3.35) and the definition for the sound velocity. C (Eq. (3.18)), have also been used. Since only materials are considered in which the sound velocity increases with pressure, it follows that

$$
\mathrm{dS}_{1}>0 \text {. }
$$


The term in the brackets of Eq. (3.39), $\left(\frac{u_{s}-u_{p}}{c_{1}}\right)^{2}$, is the absolute value of the slope of the line connecting the initial and final states in the P-V plane and is called the Rayleigh line. Since the end state, $\left(P_{1}, v_{1}\right)$, is arbitrary, it follows that entropy increases monotonically with pressure and the Hugoniot lies above the adiabat passing through the initial state, $\left(P_{0}, V_{0}\right)$, as can be seen in Fig. 3.6. It can also be shown that for weak shocks, the entropy increase is third-order in the compression. 57

Three final points about the Rayleigh line are worth discussing before proceeding on to the final two sections of this chapter. If the first and second Hugoniot relations are combined, an expression for the shork velocity is obtained,

$$
u_{s}=v_{0} \sqrt{\frac{P-P_{0}}{v_{0}-v}}
$$

which states that the shock velocity is directly proportional to the square root of the slope of the Rayleigh line. Since the curve has a positive second derivative, it follows that shock velocity is an increasing function of pressure. The next point is illustrated by considering the third Hugoniot relation. The internal energy increase in a shock compressed material is represented by the area under the Rayleigh line between the initial and final states. The energy increase due to an isentropic compression is represented by the area under the curve, $\mathrm{S}_{0}=$ const., bounded by the initial and final volumes. Therefore, the area between the Rayleigh line and isentrope (shaded region in Fig. 3.6) 


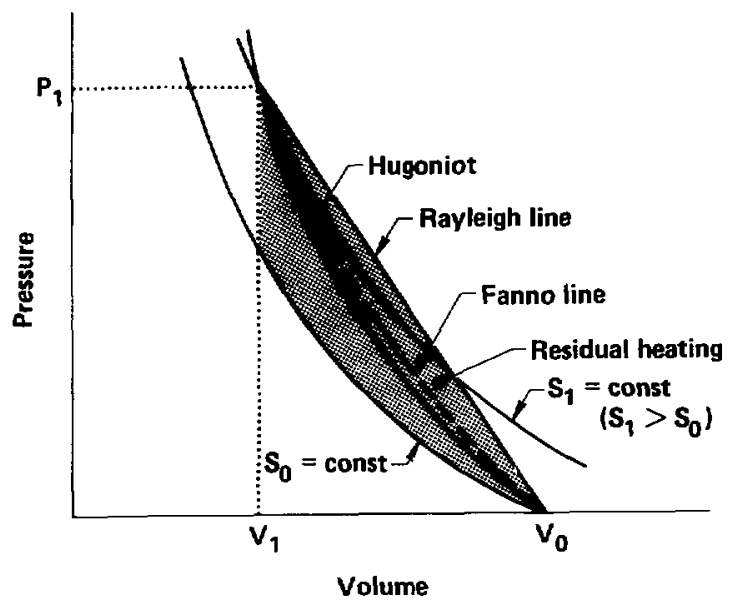

Fig. 3.6 EOS for a hypothetical material showing relative positions of the Hugoniot and adiabats for two values of the entropy. The Rayleigh and Fanno lines are also included.

represents the entropy increase or irreversible heating accompanying a shock compression process. The final point regarding the Rayleigh line is that it represents the flow trajectory in the $P-V$ plane above the equilibrium surface for a material in which heat conduction is the dominant dissipation mechanism. ${ }^{61,62}$ This can be seen by considering the momentum equation (Eq. (3.30)) for $n_{L}=0$,

$$
p+\rho v^{2}=p_{0}+p_{0} v_{0}^{2}
$$

This expression relates the intermediate states in the shock region as well as the equilibrium states. A more familiar expression for the shock pressure can be obtained by combining Eq. (3.41) with Eq. (3.31)

$$
P=p_{0}+p_{0} v_{0}^{2}\left(1-\frac{v}{v_{0}}\right) .
$$


The point describing the state of the material within the shock region goes from the initial state $\left(P_{0}, V_{0}\right)$ in the $P-V$ plane to the final state $(P, V)$ along the Rayleigh line. The states along the Rayleigh line can be reached from each other by continuous changes due to heat transfer effects, exclusively.

Now suppose there is viscosity with no heat conduction (that is, $\kappa=0$ ). The entropy equation (from Eq. (3.30)) becomes

$$
\text { pvT } \frac{d S}{d x}=n_{L}\left(\frac{d v}{d x}\right)^{2}
$$

Which shows that the wave entropy increases monotonically from the initial value, $S=S_{0}$, to the final entropy value, $S=S_{1}$, and is contained with in the two adiabats $s_{0}$ and $s_{1}$ (Fig. 3.6). Since the adiabats are convex downward $\left(\left(\frac{\partial^{2} p}{\partial v^{2}}\right)_{S}>0\right)$, the trajectory, represented by the dashed line in Fig. 3.6, lies entirely below the Rayleigh line. The trajectory is termed the Fanno line. ${ }^{62}$ The states along the Fanno line can be reached from each other by continuous changes due to frictional or viscous effects. Since it has been demonstrated that viscous and themal contributions are comparable, it is likely that the shock process proceeds along some path between to these two special cases. ${ }^{62}$

\subsection{Distended Materials}

Shock compressing materials at various initial densities reveais additional thermodynamic information. The material could be porous like a foam or simply a fluid with a less efficient packing arrangement. Let 
$v_{0}$ and $v_{00}$ denote the initial volumes of the standard and distended materials, respectively, where $v_{00}>v_{0}$. Suppose the distended material is slowly compressed to its standard volume, $v_{0}$. This will proceed along a nearly horizontal line (Fig. 3.7) since negligible resistance to deformation is assumed. This compression will close voids or break the weaker intermolecular cross-linking that inhibits efficient packing. Once the material is compressed to its standard volume, the bulk modulus becomes finite and further compression follows the adiabat starting from $v_{0}$.

The Rayleigh line for the distended material, however, is a straight line connecting the initial, $\left(P_{0}, V_{00}\right)$, and final, $\left(P_{1}, V_{1}\right)$, states. A consequence of the large area between the Rayleigh line and the isentrope is an increase in the temperature of shock compressed distended materials over their more dense counterparts. This is shown by the shaded region in Fig. 3.7. The large contribution of the thermal component to total pressure is demonstrated by the anomalous Hugoniot curve for some highly porous materials which show final volumes greater than the preshocked volume. ${ }^{61}$ The adiabat, which characterizes the repulsive forces between molecules, can be determined by comparing the Huqoniots of material with equivalent composition and differing starting densities. The relative contribution from tne thermal and elastic components can be determined by shock compressing the standard and distended materials over a range of final volumes and mapping out an EOS such as the Grüneisen form. The opportunity to observe this phenomenon provided the motivation measuring the $\mathrm{EOS}$ for $1-$ butene $\left(\mathrm{CH}_{2}=\mathrm{CH}-\mathrm{CH}_{2}-\mathrm{CH}_{3}\right)$ and comparing it with that of polybutene $\left[\left(\mathrm{CH}_{2}-\mathrm{CH}-\mathrm{CH}_{2}-\mathrm{CH}_{3}\right)_{n}\right]$. 


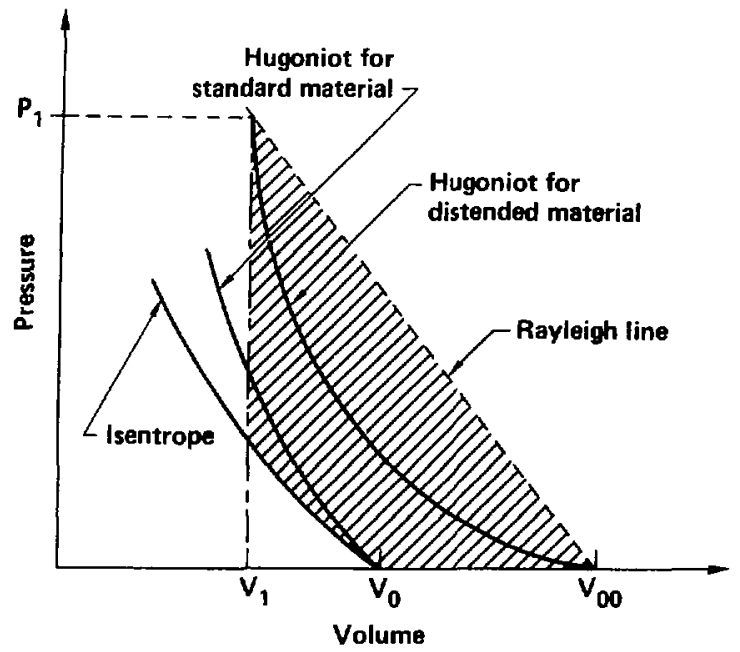

Fig. 3.7 Hugoniots for two similar materials with differing starting densities.

\subsection{Shock Impedance Hatching}

Shock impedance matching is a technique for obtaining the mass velocity, $U_{p}$, from the initial material properties and measured kinematic parameters. In an experiment to measure the Hugoniot EOS of a material, the experimenter measures one or more of the variables ( $P$, $\left.U_{p}, U_{s}, V\right)$ and uses the Hugoniot jump conditions to determine the others. Consider Eq. (3.35) for an initial velocity, $U_{0}=0$,

$$
P-P_{0}=\rho_{0} U_{S} U_{p}
$$

The shock velocity can be measured while the remaining unknowns (such as pressure and particle velocity) are determined using impedance matching 
techniques. The quantity $\rho_{0} U_{s}$ is called the impedance, $Z$, of the material and is a property of the material.

The $P-U_{p}$ plane is a convenient place to relate the impact of materials because of the continuity of pressure and mass velocity at the interface of two materials in contact. Consider the impact of two dissimilar bodies, where body $I$ is moving to the right with velocity $U_{1}$ and strikes body $l 1$ which is initially at rest. Suppose the EOS for material I, called the standard, is known. For many materials (particularly metals). a linear relation has been discovered to exist, over a wide pressure range, between $U_{s}$ and $U_{p}$,

$$
u_{s}=c+S u_{p}
$$

The relation is empirical and the value of the coefficient, $C$, approaches the sound velocity for decreasing shock strength. The materials used as a standard in these experiments are aluminum and tantalum for which the $U_{p}-U_{s}$ relation is known to accuracies greater than $7 \%$.

Upon impact, the conditions of particle velocity and pressure continuity are established by the formation of a shock front in each material propagating in opposite directions. Let $C_{7}$ and $S_{1}$ be the known coefficients of the impactor. The impactor is moving to the right at velocity $U_{I}$, so its Hugoniot relation (Eq. (3.35)) will be translated in the $u_{p}-p$ plane along the $u_{p}$-axis by a distance corresponding to a particle velocity in the lab frame and reflected about the P-axis (curve B, Fig. 3.8), The Hugoniot becomes 


$$
P_{1}=p_{1}\left(U_{I}-U_{P_{1}}\right)\left[C_{1}+S_{1}\left(U_{1}-U_{P_{1}}\right)\right] .
$$

The Hugoniot for the "unknown" target material (curve A, Fig. 3.8) is simply

$$
P_{2}=\rho_{0} U_{S} U_{P_{2}}
$$

The continuity conditions require $\mathrm{P}_{1}=\mathrm{P}_{2} \equiv \mathrm{P}_{\mathrm{H}}$ and $\mathrm{U}_{\mathrm{P}_{1}}=\mathrm{U}_{\mathrm{P}_{2}} \equiv \mathrm{U}_{\mathrm{P}_{\mathrm{H}}}$ and thus determine $U_{P_{H}}$. The Hugoniot relations can then be used to deteninine the final energy, volume and pressure. The Hugoniot can be mapped out to any desired degree of detail by repeating the experiments over a series of impactor velocities and densities.

Two practical limitations restrict the seemingly straightforward determination of the Hugoniot EOS of a sample. One is the upper velocity in accelerating a projectile with some well defined geometry. Impositions

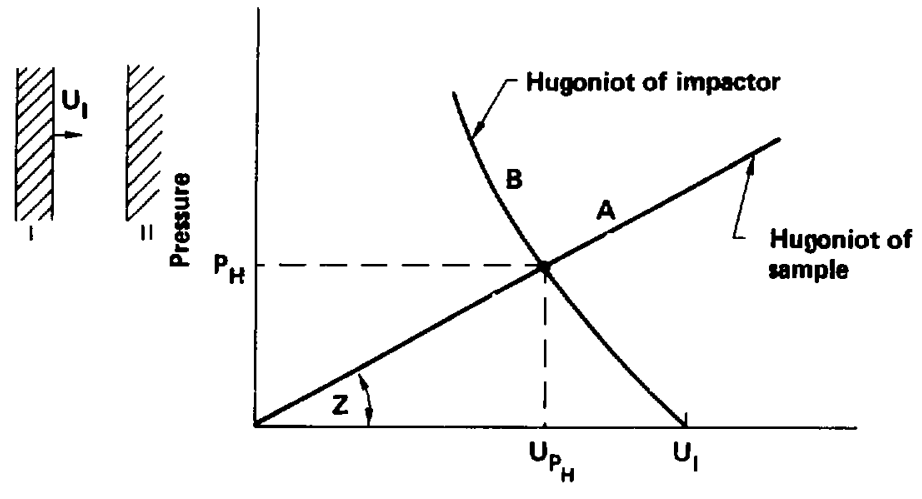

Particle velocity

Fig. 3.8 Impedance matching the impact of two dissimilar materials in $U_{p}-P$ space. 
generated by this restriction were not considered serious for the range of pressures sought in these studies. A device is described in the next chapter that can accelerate a $20 \mathrm{~g}$ projectile to velocities up to $B$ $\mathrm{Km} / \mathrm{sec}$ and produce dynamic pressures in liquid nitrogen samples in excess of $600 \mathrm{kbar}$. Another more subtle but restrictive problem concerns rarefactions. The property of materials to allow the formation of a shock wave is also responsible for the formation of rarefactions or release waves. Impactors and samples are actually finite in extent, both laterally and in thickness. When the shock reaches the back surface of the impactor the impedance mismatch of the impactor material and the vacuum produces a rarefaction in the opposite direction. The rarefaction moves with the sound velocity of the shock compressed region in the same direction as the shock wave. Shock wave stabflity requires the magnitude of sound velocity plus the particle velocity to exceed the shock velocity, the consequence of which is that the shock wave will eventually be attenuated by the catch-up wave.

Deviations from ideal uniaxial strain are another problem and result from the finite lateral extent of the materials. Release waves propagate from the edges and eventually compromise the desired ideal conditions of uniaxial strain. These problems can be avoided with a judicious croice in Experimental sample gometries. Consider the side rarefaction problem (Fig. 3.9). It can be shown that the region of sound f propagation forms an angle, $\alpha$, with respect to the point where the rarefaction begins and is given by 


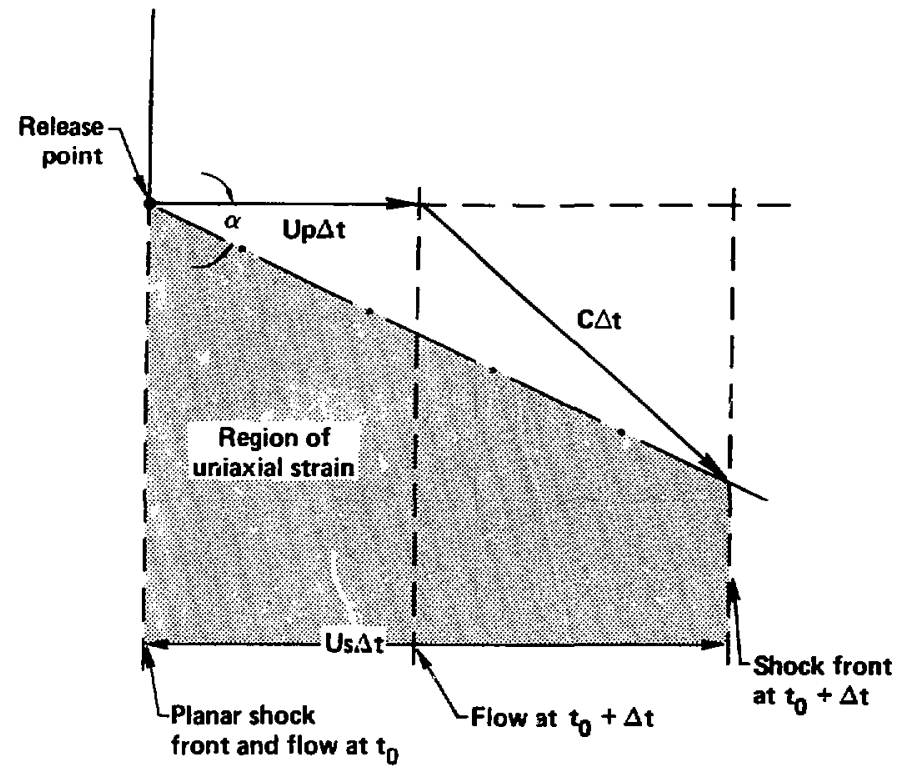

Fig. 3.9 Stde rarefaction originating at a release point.

$$
\tan a=\sqrt{\left(\frac{c}{U_{s}}\right)^{2}-\left(\frac{U_{s}-U_{p}}{U_{s}}\right)},
$$

where $C$ is the sound velocity in the shocked material. ${ }^{63}$ An upper limit to a can be determined by calculating the adiabatic sound velocity based on the slope of the Hugoniot in the shock compressed state of the sample. This has been done for the samples considered in this thesis and gives $a \leq 45^{\circ}$. This stipulation also provides a boundary condition which must be considered in the final design of an experiment. 


\section{CHAPTER 4. EXPERIHEHTAL METHOD}

\subsection{Introduction}

To induce the high dynamic pressures and temperatures discussed in the previous chapters, flat plates of aluminum or tantalum were accelerated by a two-stage light-gas gun to velocities up to $8 \mathrm{Km} / \mathrm{s}$.* The plates impacted an instrumented specimen and induced a shock wave with the properties accurately controlied and recorded. A variety of parameter measurements was made for the shock compressed state of the material from which either Hugoniot EOS or electrical conductivity data were determined.

Section 4.2 will describe essential features and principle of operation of the two-stage gun. Section 4.3 detalls the target geometry and attached sensors, followed by Section 4.4 which covers the overall diagnostic plan. The final four sections include sample preparation, temperature control, particle yelocity determination, and a summary of the sequential procedure followed in a typical experiment.

\subsection{Two-Stage Light-6as Gun}

The gun used to accelerate the impactor is an accelerated-reservoir light-gas gun with a launch tube bore diameter of $29 \mathrm{~mm}$. The gun maintains a reasonably smooth pressure at the base of the projectile

\footnotetext{
* Escape velocity from the earth's surface is $11 \mathrm{~km} / \mathrm{sec}$.
} 
during the launch thus eliminating the problems accompanying excessive impactor deformation and heating.

Figure 4.1 shows the layout of the gun and target chamber which consists of the following components:

1. Powder chamber.

2. Pump tube, $89 \mathrm{~mm}$ I.D. by 10 meters long.

3. Accelerated-reservoir high-pressure coupling.

4. Launch tube, $29 \mathrm{~mm}$ I.D. by 9 meters long.

5. Instrumented target chamber and target.

Prior to firing, a few kilograms of $M-6$ gun powder are placed in the breach and the pump tube is filled with about 60 grams of hydrogen gas. A rupture valve is located on the down-stream end of the accelerated reservoir to contain the gas. The target chanber and launch tube, up to the projectile, are evacuated to spproximately $10^{-2}$ torr to eliminate the effects of frictional heating of the projectile and

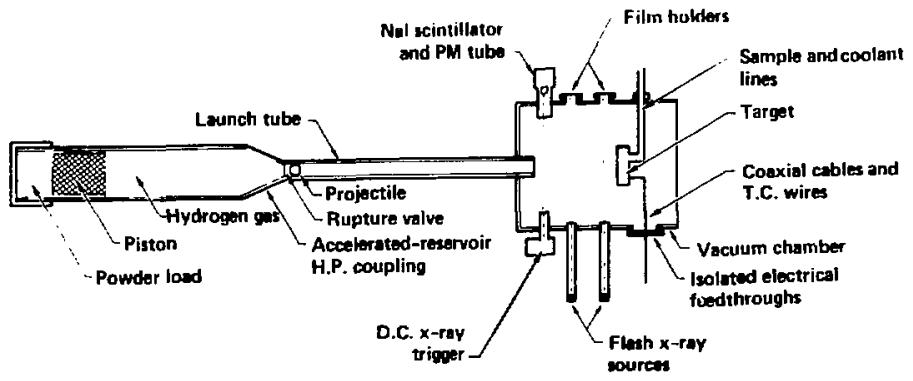

Fig. 4.1 Layout of the two-stage, light-gas gun and target chamber. 
preshocks in the target. A plastic cylinder, called a sabot, carries the impactor plate. The impactor is hot-pressed into the sabot which carries it in a fixed position and seals the driving gas behind the projectile.

Once the powder is ignited, the expanding gases accelerate the piston toward the accelerated-reservoir high-pressure coupling. At a critical pressure the diaphram ruptures and allows the expanding hydrogen gas to accelerate the 20 gram projectile toward the target. Shortly after the projectile enters the target chamber, it interrupts a continuous $x$-ray beam focused on a NaI scintillator detector. The time at which this occurs provides a timing reference to sequence future events such as firing the flash $x$-ray tubes and the current pulser used in conductivity measurements.

The amount of powder, rupture valve pressure threshold and light-gas quantity are determined by the projectile velocity required. Generally, impactor velocities can be predetermined to an accuracy of $5 \%$. Flash $x$-rays 1 and 2 are adjusted to pulse as the projectile crosses their paths, which is timed from a knowledge of the chosen projectile velocity and measured positions of the flash $x$-ray tubes with respect to each other and the continuous $x$-ray trigger. Hewlett-Packard time-internal monitors (TIM) record the precise moment the $x$-rays are pulsed while film placed behind a scintillation screen, on the opposite side of the target chamber, recards the projectile position. Projectile velocities can be determined to within $0.7 \%$. 

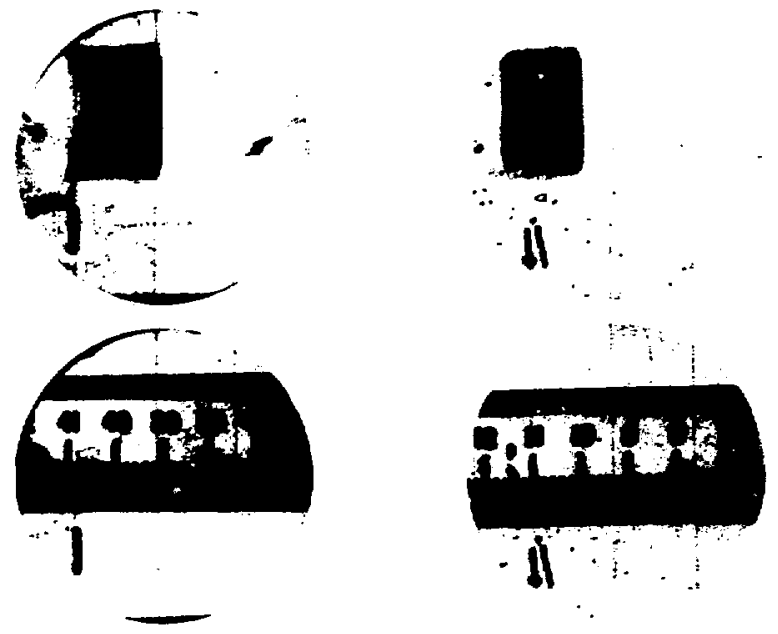

Fig. 4.2 Typical flash $x$-ray records.

Set-up pictures, with millimeter-marked rule (zero of scale located at baseplate), and shot pictures, with the projectile moving to the right, are shown in the lower and upper frames of Fig. 4.2, respectively.

The planarity of the shock wave induced in the target is dependent on the impactor flatness at impact. The impactor plate material is either $2 \mathrm{~mm}$ thick tantalum or $4 \mathrm{~mm}$ thick aluminum, each with $24 \mathrm{~mm}$ diameters. The front surfaces are machine lapped and then hand polished flat to 5 microns. EOS measurements using a 13-pin array and other independent tests confirm aluminum impactor flatness to less than 5 ns for $7 \mathrm{~km} / \mathrm{sec}$ velocities. The impactor tilt, relative to the target specimen, is dependent on launch tube uniformity and target alignment. Optical techniques allow target alignments with respect to the launch tube to approximately 0.03 degrees. 
Careful attention to the condition of the launch tube is necessary for successful firing in the higher velocity ranges. Bore uniformity of better than 200 microns over the ful1 9 meter length of the launch tube is maintained. The diameter is maintained constant to within 10 microns. Launch tubes are cleaned between each firing dind honed after an average of 20 firings. With cryogenic samples it is generally possibie to maintain a schedule of one shot per day with a single shift which includes the physicist, two mechanical technicians and one electronic technician.

A more detailed description of the two-stage light-gas gun, which is maintained and operated by the H-Division high pressure experimental group at LLNL, can be found in Refs. 7, 64-68.

\subsection{Iargets}

The target bodies contain the sample and provide a secure mounting for the pin block, thermocouples and electrode assembly. The target bodies for the cryogenic shots possess an annular region surrounding the sample cavity. Liquid nitrogen fills the cavity for the liquid nitrogen and oxygen conductivity shots, and flowing cold nitrogen gas fills the chamber for the liquid 1-butene EOS experiments. In either case, the large thermal inertia of the aluminum target body in contact with the coolant provides a stable means for temperature control. The basic body design is the result of several years of refinements and has been used in EOS, pyrometry and Raman experiments using a large variety of liquids. Figures 4.3 and 4.4 are schematic, cross sectional views of the standard 


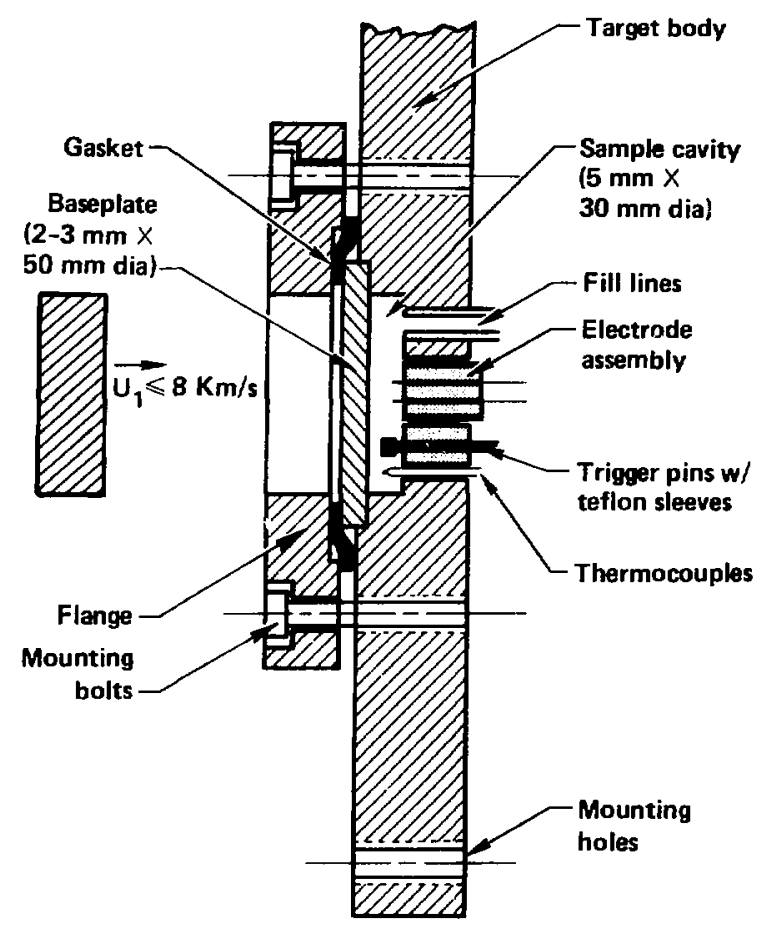

Fig. 4.3 Cross sectional view of standard target used for benzene conductivity measurements.

(room temperature) and cryogenic targets set up for a conductivity measurement.

The bodies are machined from aluminum and measure about $15 \mathrm{~cm}$ in dlameter. A $3 \mathrm{~cm}$ diameter cavity is machined in the standard target to a depth of $5 \mathrm{~mm}$. The ratio of diameter-to-depth is sufficient to insure a condition of uniaxial compression. The mounting flanges for the baseplates and target bodies are an aluminum alloy (6061) easy to machine 


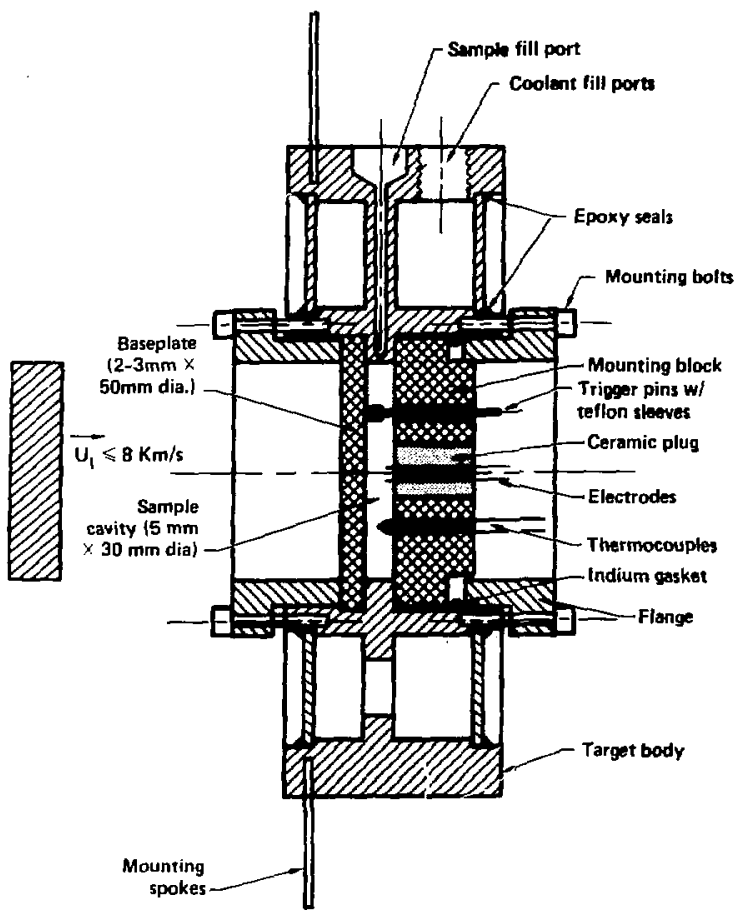

Fig. 4.4 Cross sectional view of cryogenic target used for 1-butene EOS, and oxygen and nitrogen conductivity measurements. (Coolant vent not shown).

and still thermally compatible with the softer, pure aluminum (1100F) used for the mounting blocks and baseplates.

The baseplate thicknesses are measured using a Bausch and Lomb metric optical gauge and are parallel to within 2 microns. Their densities are determined by a buoyancy method to an accuracy of $0.1 \%$. 
The electrode assembly (Fig. 4.5) consists of a layered composite of machinable glass ceramic (Maco $B$ insulating spacers and 25-50 micron thickness copper or stainless steel foils machined to 5 micron tolerance and platinum plated to prevent oxidation. The foils are chosen in order to minimize perturbing effects to the sample. The transition in lateral extent, from 1 to $10 \mathrm{~mm}$, at the interface between the sample cavity and its wall better defines the position of the shock front with respect to the electrode base and marks the signal of interest in a measurement. The large-width base allows for easier attachment of wires and decreases resistivity from sample circuitry. The close tolerance on the foils is accomplished using a wire electrical discharge machining (EDM) tool. The tool leaves a $0.005 "$ radius on the corners which prevents excessively high local electric fields. Figure 4.6 is an enlarged view of the foils and it should be noted that unly the tips, which measure $1 \mathrm{~mm}$ by $1 \mathrm{~mm}$, extend into the sample. The spacers keep the

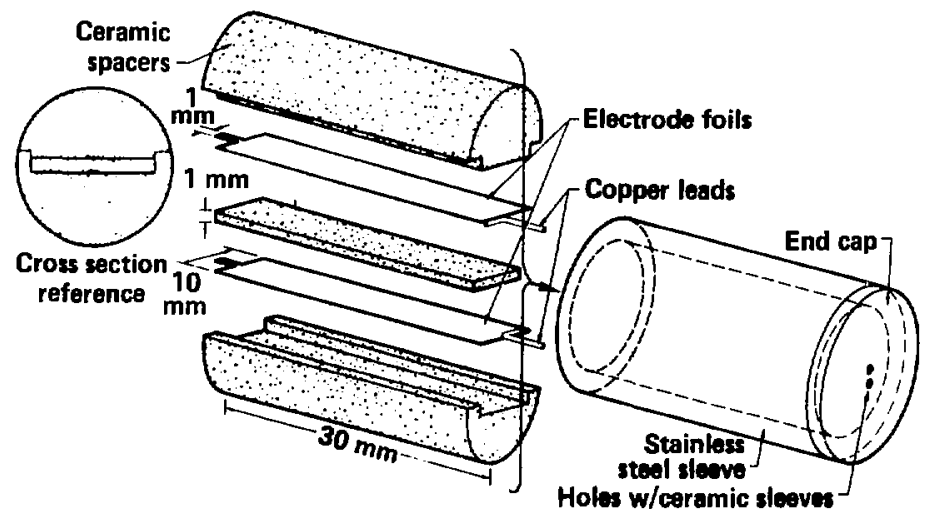

Fig. 4.5 Exploded view of electrode sandwich. 

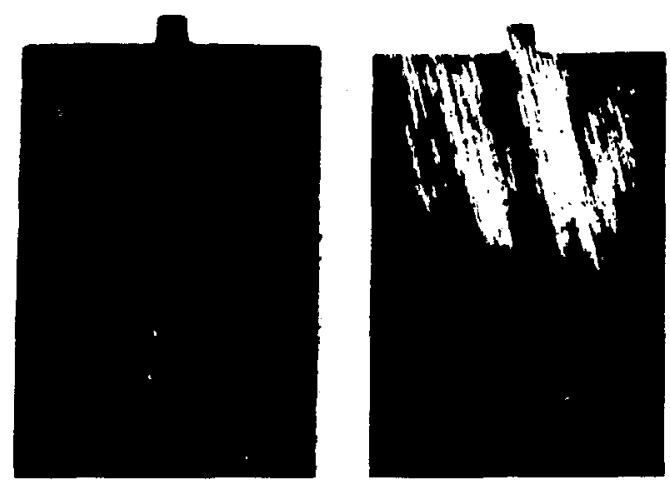

Fig. 4.6 Photograph of electrode foils enlarged $4 x$.

foils paralle 1 and separated a distance of $1 \mathrm{~mm}$. A stainless steel sleeve, which is used to improve the vacuum sea 1 integrity between the sample and target chambers, is pressed ( 50 micron interference) into the pin block prior to inserting the electrode plug. Varian Torr-Sea $\mathbb{P}$ is used as a bonding agent because of its low vapor pressure and excellent thermal cycling properties. The depth the electrode plug is inserted into the sleeve is determined by the baseplate-to-electrode distance requirement and must be determined for each case. The separation is important because an optimum balance must exist between catch-up rarefactions originating from the impactor back surface and the baseplate proximity to the electrode tips. A one-dimensional, Lagrangian elasticplastic hydrodynamic code KOVEC ${ }^{69}$ is used to aid the design considerations with regard to the above mentioned phenomena. Any potential problems with side release waves are avoided by positioning all probes and detectors well within a conservatively assigned region $45^{\circ}$ from the release point (See Section 3.6). 
Surfaces which will come in contact with the sample are cleaned using a caustic bath followed by a $\mathrm{HCl}$ bath and deionized weter rinse. Target body surfaces are cleaned with alcohol just prfor to assembly.

For electrical conductivity measurements, four holes are bored in the pin block; two opposing holes are bored on an $18 \mathrm{~mm}$ diameter circle for the trigger pins and two opposing holes, $90^{\circ}$ from the pin holes on the same circle, are bored for copper/constantan thermocouples. Teflon sleeves keep the outer shorting pin shafts electrically isolated from the target body. This is to prevent ground currents since the pin conditioning circuit is electrically common to the gun tank. This consideration will be discussed in the section addressing diagnostics. The pins and thermocouples are also positioned and vacuum-sealed with epoxy in the mounting block. The pins trigger the faster-sweep oscilloscopes used in recording the voltage drop across the electrode/sample combination and have a time resolution of about 5 nanoseconds which is sufficient. These pins are the coaxial-type consisting of an inner conductor separated by an insulating shim from a grounded outer tube and end-cap. The shock transit through the Insulating end-cap closes an RC circuit which appears as a pulse on an oscilloscope. The uncertainty in the gap separation of these pins excludes their use for EOS measurements but their coaxially-sealed design makes them attractive as triggers for the conductivity measurements since their shielding reduces or eliminates interactions between the triggering and measuring circuits.

The detector package for the EOS targets (Fig. 4.7) consists of a 


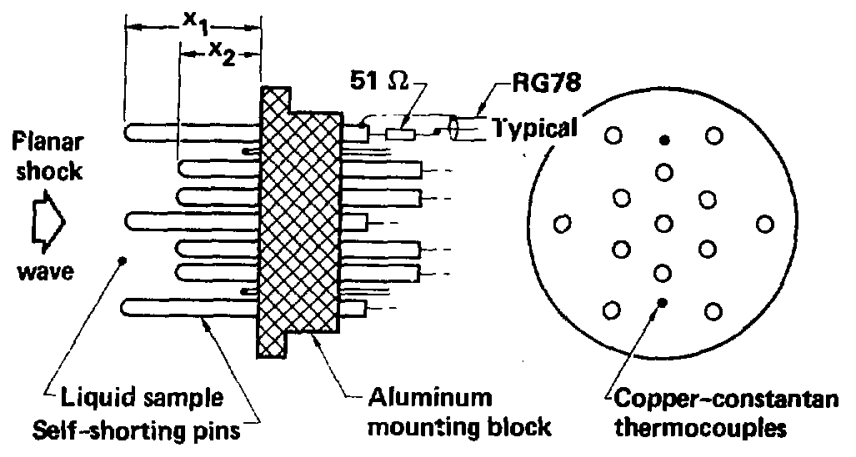

Pin block Pin block end view

Fig. 4.7 Pin and thermocouple positions for an EOS measurement.

set of 13 pins and two copper/constantan thermocouples epoxied in place. These pins differ from the pins used in the conductivity shots. The pins for the EOS measurements are self-shorting coaxial pins consisting of a central, anodized aluminum wire surrounded by an outer grounded tube. Their subnanosecond closure time allows the position of the shock front to be determined accurately as it passes through the sample. For multiplexing purposes, a $51 \mathrm{ohm}$ terminating resistor is connected in series with the pin and ground to suppress voltage reflections in the cable once the pin is shorted. These pins are supplied by Specialty Engineering. Associates and are described in greater detail in Ref. 64. The pins located on the first plane are usually $0.3 \mathrm{~mm}$ from the baseplate and located on an $18 \mathrm{~mm}$ circle. Thr pins on the second plane are usually $2.5 \mathrm{~mm}$ from the baseplate and located on a $10 \mathrm{~mm}$ circle. Their heights are determined optically to an accuracy of 2 microns.

The cell constant, defined in the following section, is a geometric 
correction factor accounting for the departure from ideal one-dimensional electric field lines across the electrodes. The electrodes are immersed into an electrolyte solution of known resistivity then pulsed using the constant-current circuit, also described in the next section. The voltage deflection, recorded on a fast-sweep oscilloscope (100 ns/div), is compared to deflections produced by a set of known precision resistors. This widely used technique, termed "electrolytic sinulation," gives the resistance and determines the cell constant. Heasurements on selected cells have bien repeated several weeks apart to cest degradation of the electrode and reproduce the cell constant to within 10\%.

The fill lines for the benzene target are Polyf $1 \mathbb{B}_{\text {tubing and }}$ pass from the target to liquid feed-throughs on a target chamber access port. The fill and coolant lines for the cryogenic targets are stainless steel bellows with a glass transition placed between the target and chamber access port. This precaution insures target/target chamber electrical isolation.

The mounting gimbal positions the target in front of the gun muzzle and is also electrically isolated from the target. This is accomplished by passing the mounting spokes through insulating spacers, located on the ginbal.

The signal cables, origfnating from the rear of the target, pass through electrically isolated BNC vacuum-quality feed throughs on the target chamber. Connections between electrodes, shunt and currentitiniting resistors are short $(\sim 1 \mathrm{~cm})$ to prevent inductive paths or 
pick-up loops. A copper shield is placed around the electrical package on the target and then filled with a silicone elastomer. This precaution is intended to prevent dielectric breakdown due to hot ionized gases from the gun that precede the projectile.

\subsection{Diagnostics}

Due to the short time scale of a dynamic experiment, the sample resistances must be measured over a $100 \mathrm{~ns}$ interval ( $1 \mathrm{~ns}=10^{9}$ s) with a resolution on the order of $5 \mathrm{~ns}$. The measured resistance, $R$, of a sample is related to the resistivity, $\rho$, the exposed area, $A$, of the electrodes, and the electrode-separation, $\ell$, by

$$
R=\rho \frac{2}{A} f,
$$

where $f$ is the dimensionless fringing field correction which may also be time-dependent. If the time dependence of the conductivity signal is assumed to be dominated by the time dependence of the area of conducting fluid swept out between the electrodes, then it follows that the scope deflection appears as $t_{0} /\left(t+t_{0}\right)$. starting where the shock wave reaches the electrode tip and finishing where the shock wave has traversed the complete length of the electrode (1 $\mathrm{mm}$ length). For a typical shock velocity of $10 \mathrm{~mm} / \mu \mathrm{s}$, the time of traversal is on the order of $100 \mathrm{~ns}$. The signal character changes once the shock wave completely traverses the electrodes and reflects of the Maco $\beta$ plug. This change provides a timing reference to determine the final signal position. The position of the catch-up wavc and baseplate, at the instant the shock wave reaches the Maco $@$, is determined by a KOVEC calculation for the relevant 
geometry. Impactor, baseplate and sample thicknesses are designed so that the rarefaction, which originates from the impactor rear surface, does not enter the sample during the experiment. The sample must also be sufficiently thick to keep a safe distance between the electrode tips and baseplate. Independent tests have determined that a $1 \mathrm{~mm}$ separation between the baseplate and electrode tips is sufficient.

The distance between the target chamber and the control/monitor room requires long cable runs $(30 \mathrm{~m})$. This creates potential problems including increased inductance and transmission line loss. Regarding inductance, circuits leads are kept short; for line loss, low-loss Foam-flex ${ }^{(1)}$ cables are used for the signal cables. Ground looping and radiative pickup were two additional problems encountered. These prcblems were reduced or eliminated by using shielding and isolated differential circuits. Huch of the support diagnostics of the two-stage gun is common-ground to the target chamber instead of differential mode and so care must be taken to insure isolation. During the experiment the ground potential of the entire gun changes. This change is comparable to the measured voltages of interest and can nullify an experiment. So, care is taken to isolate the probes and associated electrical devices from the gun tank. This has proven successful. Two additional reasons prompted the use of a differential system: The baseplate is not biased so that it could become an alternate current path (voltage-divider effect) as it approaches the electrodes, and any polarization noise pulse, which can accompany the shock compression of some materials, is electrically cancelled in a true differential system with a transverse geometry. 
Two basic variations of a Reimers circuit are used to measure the final sample resistance. 25 They are the constant-voltage circuit (Fig. 4.8a), which is an effective circuit for measuring resistances above 10 ohm, and the constant-current circuit (Fig. 4.8b) which is the preferred system for measuring lower resistances. In the constant-voltage circuit. a capacitor is charged through two large ballast resistors $(51 \mathrm{~K} \Omega$ ) and a differential power supply. When the shock reaches the electrodes, a current path is established and the circuit becomes equivalent to switch

\section{Constant voltage}
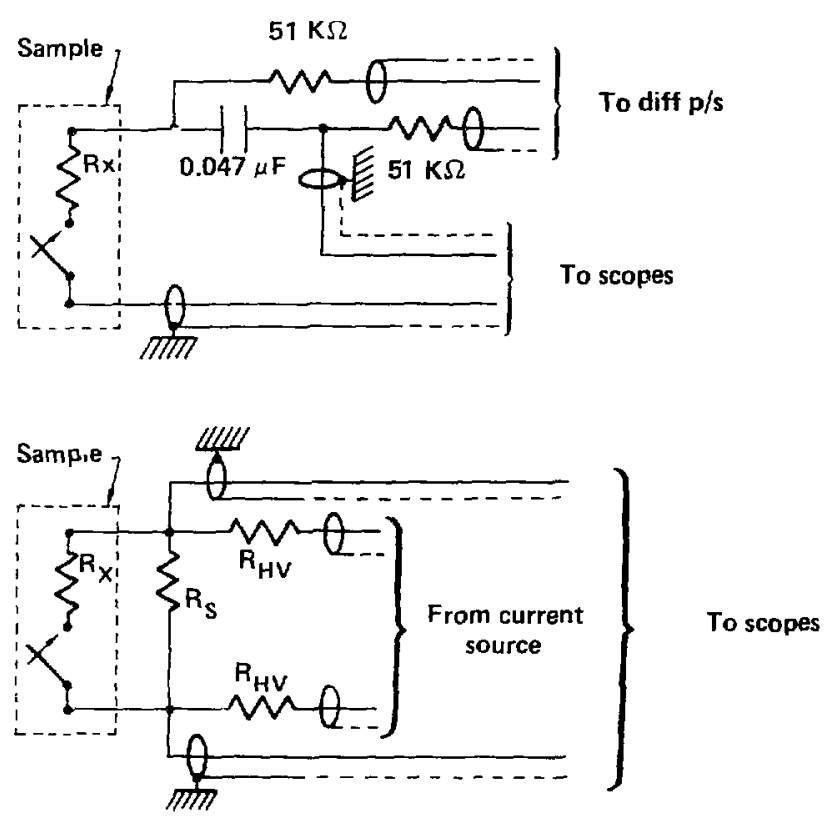

Constant current

Fig. 4.8 Fquivalent electrical circuits for (a) constant-voltage and (b) constant-current voltage measurements. 
closure in an RC circuit. The signal is transmitted along the coaxial lines to differential amplifiers and fast-sweep (50-700 ns/division) oscilloscopes. About seven oscilloscopes, in a parallel arrangement set at progressively lower sensitivities, monitor the signal strength. A photographic record is taken of the signal on the fast-sweep oscilloscopes, which are triggered by the shorting pins. The signa? deflection, which appears on one or more of the scopes with the matching sensitivity, is compared with deflections produced by a set of standard precision resistors in combination with a fast rise-time (5 ns), low resistance $(\approx 1 \mathrm{ohm})$ silicon-control rectifier switch (SCR).

Typically, the potential, $v_{0}$, set for the constant-voltage circuit was 100 volts. The final voltage deflection, $v_{x}$, measured across a terminating resistor of value $R_{2}$ is related to the sample resistance, $R_{x}$, through

$$
v_{x}=\frac{v_{0}}{\frac{R_{x}}{R_{z}}+1} .
$$

A calibration procedure using a set of standard resistors was preferred to the absolute voltage calibrations of each oscilloscope. A record of deflection, $y_{i}$, is produced by the set of resistors, $R_{i}$, and is plotted as a function of $x_{i}$ where

$$
x_{i} \equiv \frac{v_{0}}{\frac{R_{i}}{R_{z}}+1} .
$$

A linear regression analysis is performed relating 


$$
y_{i}=a x_{i}+b
$$

Equation (4.4) is inverted to obtain the final sample resistance, $R_{X}$, from the measured deflection, $y_{x}$. Figure 4.9 shows a signal trace for liquid nitrogen shot $\mathrm{NC}-10$ using a constant-voltage circuit. The final deflection voltage, $v_{0}$, appearing in Eqs. (4.2) and (4.3), is the difference between the baseline and conductivity signa?.

The constant-current circuit is effectively a current generator that is activated just prior to the shock wave reaching the electrode tips. This is accomplished by charging two $100 \mu$ capacitors through two large ballast resistors with a differential power supply. An SCR switch allows current to flow through the shunt and current limiters

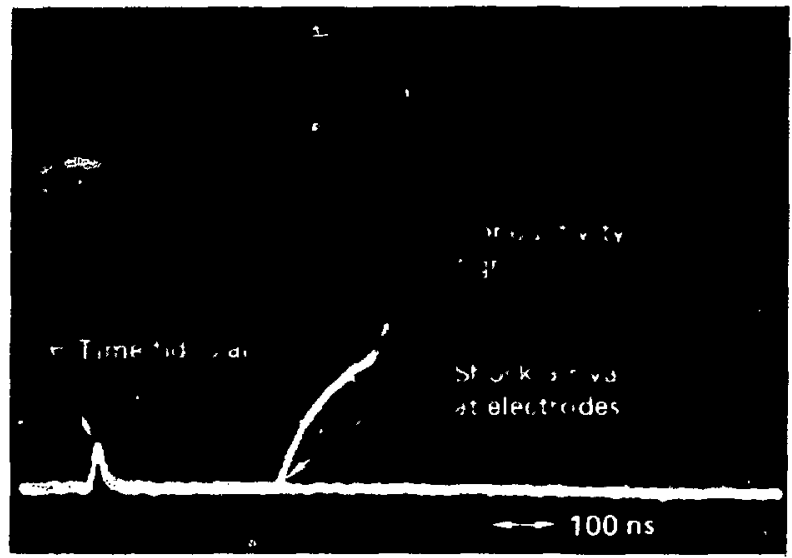

Fig. 4.9 Voltage deflection for a liquid nitrogen conductivity experiment using a constant-voltage circuit (NC-10, $P=19.1$ GPa, $\sigma=1 \times 10^{-3} \Omega^{-1} \mathrm{~cm}^{-1}$ ). 
about $30 \mu$ s before the shock wave reaches the electrodes. This is enough time to allow for the disappearance of unwanted switching transients. The current-limiting resistors, which are at least 2 orders of magnitude larger than the shunt resistors, determine the current magnitude and remain essentially constant during the experiment. Once the shock wave reaches the electrode tip, another finite resistive path is created and this behaves as a voltage-dividing device. The voltage change is recorded on a set of fast-sweep oscilloscopes. The scope sensitivity and power supply voltage are adjusted to maximize the separation between the baseline and initial signal deflection. Also, maximum sensitivity in the circuit is achieved by matching the shunt resistor value to the expected final resistance of the shocked sample. This usually requires some guess-work since the effective range of sensitivity is only 2 orders of magnitude on the resistance scale compared to the constant-voltage circuit which covers nearly 5 decades. For this reason, some experiments were performed twice in order to match the region of optimum sensitivity for the electronics to the final shock-induced conductivity.

Details of the current pulser, which is the capacitor/switching network, can be seen in Fig. 4.10. The high voltage cables (from the power supply) and ballast resistors charge the capacitors. SCRs (denoted by 2N4174) inhibit current flow until a bias is applied from a "start pulse" on the right. Transformers (desoted by APT 111-1) are used to decouple any unwanted signals which might originate from the "HV+/-" or "start pulse" terminals. The "stop pulse" circuit is used only during simulations and prevents excessive power deposition in preshot 


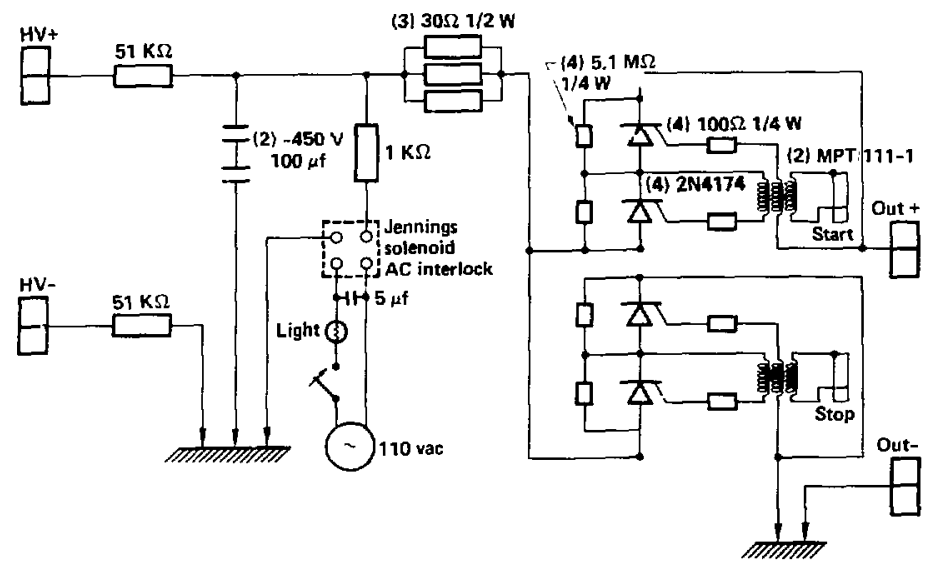

Fig. 4.10 Current pulser showing capacitor/switching circuit and interlock used in a constant-current conductivity experiment.

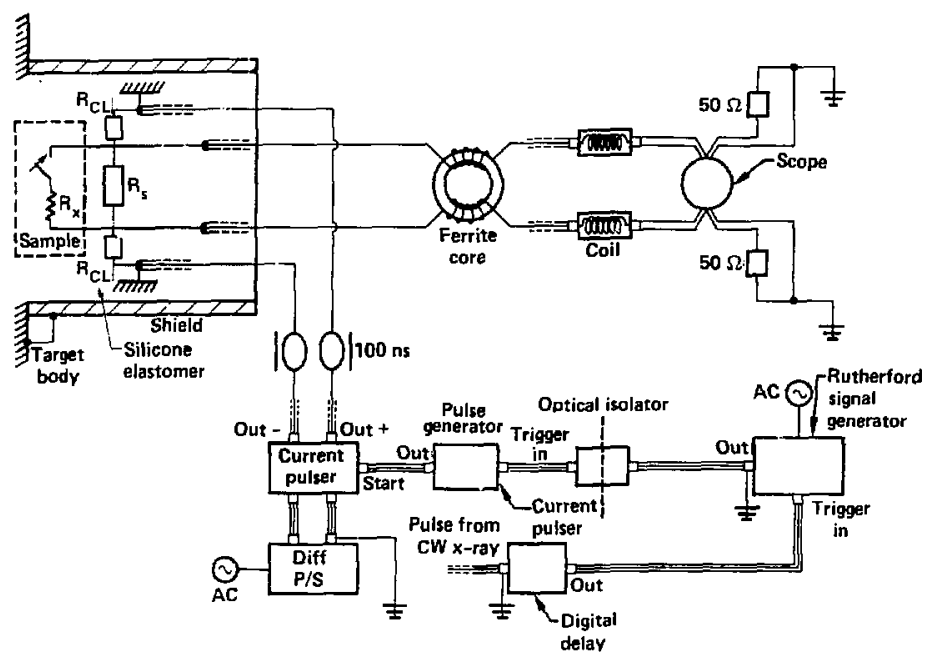

Fig. 4.11 Essential electrical elements of a low resistance measurement. 
instrumentation. Also shown in the circuit is an "AC interlock" included for safety reasons. The capacitors are discharged to ground when the $A C$ power is shut off. Figure 4.11 is an abridged depiction including only essential elements of the current source, start pulse circuit, signal runs and ground planes. Fig. 4.10 is a detailed schematic of the "current pulser" shown in Fig. 4.11. A complete description of the circuits and other variations can be found in Refs. 65, 70-74.

Figure 4.12 shows the voltage dividing characteristic of a constant-current circuit as the shack wave traverses the electrode length. The initial deflection to the left of the ramp is $v_{0}$. The

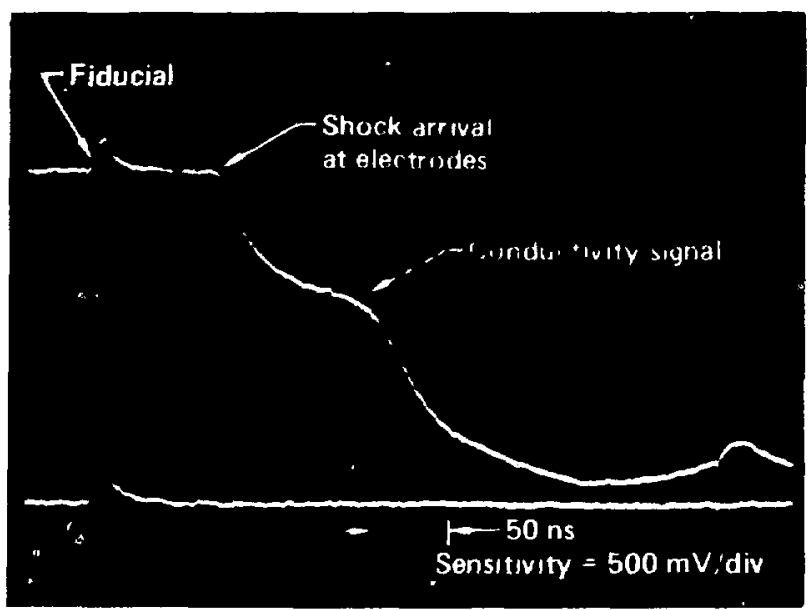

Fic $\quad 12$ Voltage deflection for a liquid nitrogen conductivity experiment using a constant-current circuit (NC-B, $P=33.8$ $\left.6 \mathrm{~Pa}, \sigma=2 \Omega^{-1} \mathrm{~cm}^{-1}\right)$. 
arrow showing the position of the conductivity signal is $V_{x}$. Since the current before and after "switch closure" is equai, the final sample resistance, $R_{x}$, is

$$
R_{x}=\frac{R_{s}}{\frac{v_{0}}{V_{x}}-1} \text {, }
$$

where $R_{S}$ is the shunt resistance and $V_{0}$ and $V_{x}$ are the oscilloscope deflections before and after shock arrival, respectively. Note the dependence on the deflection values appears as a ratio, as opposed to their absolute magnitude and that a maximum in sensitivity is obtained for $R_{x}=R_{S}$.

A schematic for the complete diagnostic system for conductivity and, to some extent, EOS measurements is shown in Fig. 4.13. The signal source and destination has been described. Recall that the projectile interrupts the $\mathrm{CW} \times$-ray beam which is used to trigger a slow-sweep ( 5 rs/division) oscilloscope and the current pulser. The trigger pulse from the $C W x$-ray is split and rerouted through a series of digital delay units which are preset to synchronize with the appropriate chronological order of events.

The pin system includes a power supply, pulse shapers, and a mixer which accepts multiple signal inputs and responds to the first signal with a conditioned output signal. The pulse shaper contains a ballast resistor/capacitor combination and a diode-to-ground to suppress signal reflections. Also included is a 10:1 attenuator which routes $10 \%$ of the pin signal (10-20 volts) to the mixer. The multiple outputs of the mixer 


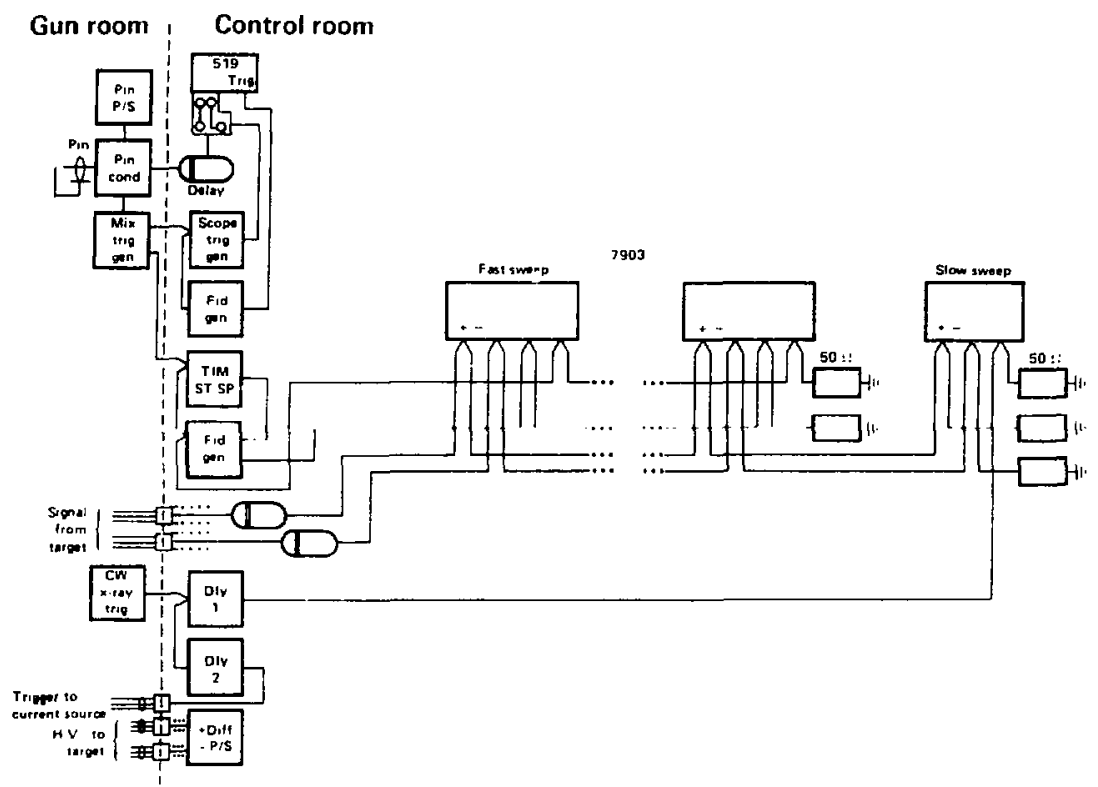

Fig. 4.13 Complete electronic diagnostic system for electrical conductivity experiments.

go to TIMs which record the $C W \times$-ray to pin closure time-intervals, to a fiducial generator and scope trigger network of the Tektronix 7903 and scope triggers, and to a fiducial generator of the Tektronix 519 oscilloscope which record subnanosecond pin signal times at ultra-fast high resolution.

The shock velocity is determined by measuring the shock arrival time for a set of pins (13) located on two planes at radii 0,5 and 9 m. Seven pins are located on the first plane, at a radii of 0 and $y \mathrm{~mm}$, and are nearest to the baseplate. The remaining pins are on the second plane, at a $5 \mathrm{~mm}$ radius. The pins have an angular separation of $60^{\circ}$ and 
a phase difference from the down-plane to $u_{i}$ plane of $30^{\circ}$. Corrections are made to the arrival times for cable lengths, differing pin heights on any one plane, shock tilt and bowing. Differences in cable lengths are measured to within 0.1 ns using an EG\&G model LE 15313-2 digital reflectometer and a Hewlett-Packard Model 5370A TIM.

A typical signal trace from a Tektronix 519 showing a fiducial and one pin each, from the first and second plane, is shown in Fig. 4.74. Separation between the two pin pulses is inferred from the superimposed calibration using a computer-assisted film reader.

Two planes are defined which represent the average pin height for

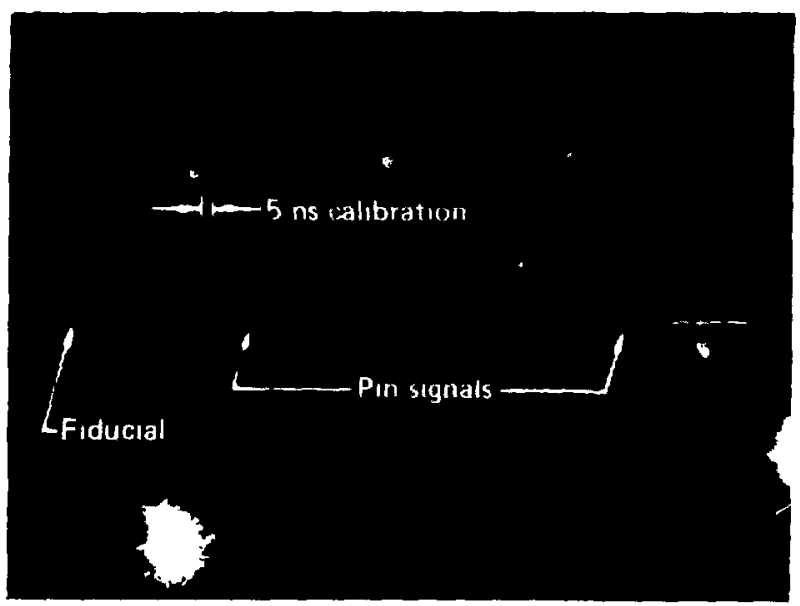

Fig. 4.14 Typical Tektronix 519 record of pin signals from the first and second plane, and timing fiducial for an EOS experiment. Also shown is a timing calibration. 
that plane. The shock arrival times for each pin on each plane are related to the arrival on that plane corrected to the average pin height of the plane using an estimated shock velocity. The corrected times (which are typically $\sim 1$ ns) are averaged for each plane,

$$
t_{j}=\frac{1}{6} \sum_{j=1}^{6} t_{j i}, j=1,2
$$

and fitted to a cosine dependence

$$
t_{j i}-t_{j}=\frac{-R_{j} \tan \theta}{U_{I}} \cos \left(\alpha_{0}+60 i\right),
$$

where $t_{j i}$ is the deviation of the $j^{\text {th }}$ arrival $t$ ime on the $j^{\text {th }} p$ lane, $k_{j}$ is the radius of the circle of the detectors on the $j^{\text {th }}$ plane, $\theta$ is the tilt of the impactor with respect to the target normal, $U_{I}$ is the impactor velocity, and $\alpha_{0}$ is the angular separation between the first point on the detector circle struck by the shock and the first detector to respond. The fitting parameters, $\frac{k_{j} \tan \theta}{V_{I}}$ and $\alpha_{0}$, give a set $\left(t_{j i}\right)_{f i t}$ and $t_{j}$ detemined from $E q .(4.6)$. The interplanar shock transit time neds to be corrected for shock bowing. A parabolic bow shape is assumed and a correction is made by calculating the arrival time difference between the center pin and first plane:

$$
\Delta t_{c}=\left(t_{c}-t_{1}\right)\left(1-\frac{R_{2}^{2}}{R_{1}^{2}}\right),
$$

where $t_{c}$ is the shock arrival time (pin-height and cable corrected) on the center pin. The interplanar shock transit time is

$$
\Delta t=t_{2}-t_{1}-\Delta t_{c}
$$


which ultimately gives the shock velocity. The standard deviation to the cosine $f i t$ is obtained by methods of standard regression analysis and is described in Refs. 7 and 66 . Shock velocities are determined to an accuracy of within $1 \%$.

\subsection{Sample Preparation and Condensation}

The specimen chamber is evacuated to a pressure of $10^{-6}-10^{-7}$ Torr using a diffusion pump. Reagent grade (Mallinckrodt, Inc.) benzene is poured into a reservoir located directly above the target outside the target chamber (Fig. 4.15). Valve $v_{1}$ is closed followed by the opening of valve $v_{2}$, then $v_{3}$ which flushes the specimen chamber with benzene while decreasing the likelihood of gas bubble formation. Finally, all valves are closed and benzene sample preparation is complete.

Specimen chambers are also evacuated for cryogenic sample transfer. In this case, however, a series of specimen fills and evacuations are done to decontaminate the system. After the final decontamination the sample chamber is filled to a pressure of $800 \mathrm{~mm} \mathrm{Hg}$ with the high purity sample gas.

Mass spectrographic analysis for each sample shows the major impurities and concentrations for $\mathrm{N}_{2}$ samples to be $0_{2} \leqq 0.1$ mole percent), $\mathrm{O}_{2}$ simples to be $\mathrm{CO}$ and $\mathrm{N}_{2}\left(\leqslant 0.7\right.$ mole percent), and $\mathrm{C}_{4} \mathrm{H}_{8}$ samples to be $\mathrm{CJ}$ and $\mathrm{CO}_{2}(\leqslant 0.7$ mole percent $)$.

Liquid nitrogen was used as a coolant for both oxygen and nitrogen 


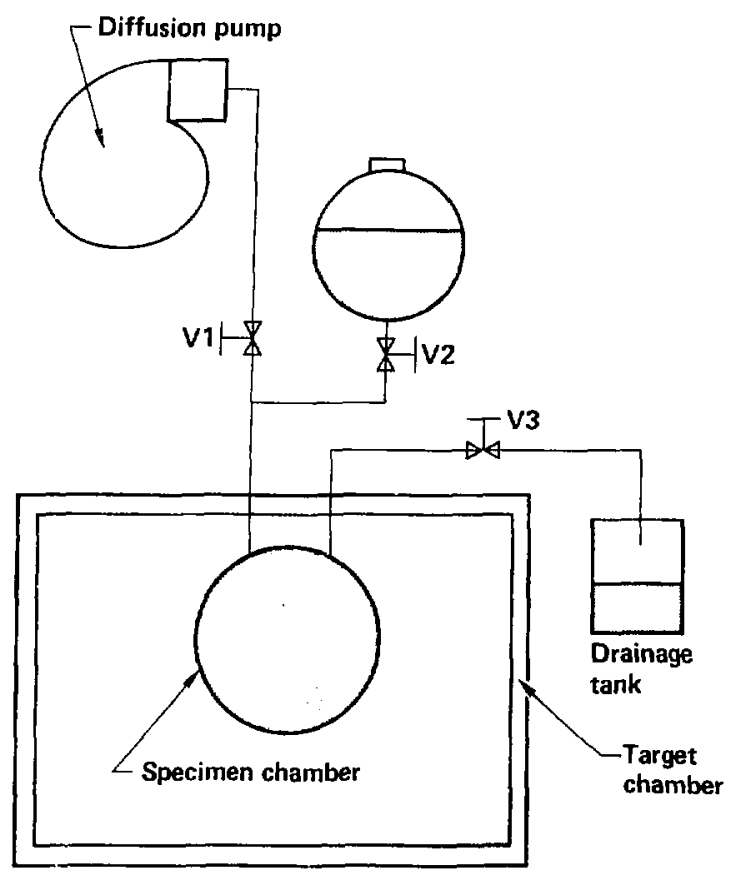

Fig. 4.15 Sample preparation system used for benzene conductivity experiment.

conductivity experiments, while cold nitrogen gas was used in the 1-butene EOS experiments (Fig. 4.16). The gas pressure in the chamber cavity was allowed to come to equilibrium with atmospheric pressure by opening the system between reservoir, specimen chamber and vent. Temperature and pressure readings fom a mercury barometer, aneroiu barometer, Heise pressure gauge (Gauge 1), and a MKS Baratron pressure meter (Gauge 2) are recorded. Valves $v 110, V 111$ and $V 112$ are closed and target cooling commences. Two different cooling methods are described in Section 4.5. Gauge 2 inditates a dramatic decrease in pressure once the temperature corresponding to the vapor/liquid saturation temperature is 


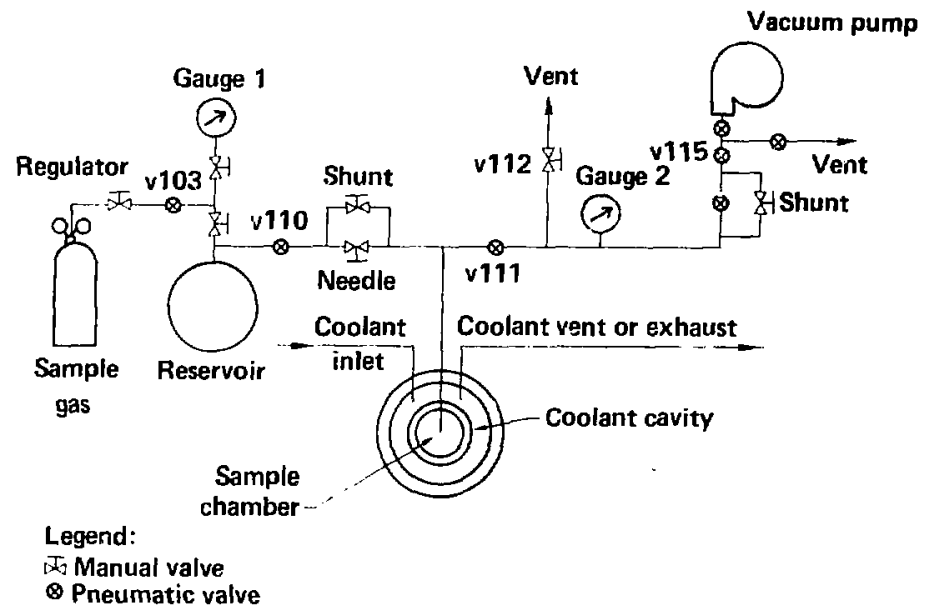

Fig. 4.16 Sample condensation system for cryogenic samples.

reached. The reservoir is filled to a pressure of approximately $1400 \mathrm{~mm}$ $\mathrm{Hg}$ (indicated by Gáuge 1) by opening valve V103. Reservoir and sample cavity volumes were approximately 4300 and $4.5 \mathrm{cc}$, respectively. Gas flow into the sample chamber is permitted by valve vilo and throttled by a needle valve which regulates the rate of sample condensation. Sample cavity pressure is maintained at approximately $800 \mathrm{~mm} \mathrm{Hg}$ to suppress hubbles. For the liquid nitrogen samples, a thermocouple calibration is done when the sample cavity is about half-filled and will now be described. The method used for thermocouple calibration on the cold gas flow system is described in Section 4.5.

Thermocouple calibration for liquid nitrogen samples is performed by allowing the pressures of the sample and conlant chambers to equilibrate. This is done by venting the sample and coolant lines to the 
atmosphere through long lines flushed with sample gas. The themocouple and pressure readings are recorded, the vent closed and condensation continued until the sample cavity is filled.

Saturation data from Refs. 75 and 76 are fitted locally to a Ciausius-Clapeyron equation of the form

$$
\ln P=\frac{-a}{T}+b
$$

where the parameters $a$ and $b$ are given in Table 4.1. The measured pressure and Eq. (4.10) provided the single-point calibration and enables a final sample temperature determination before gun fire. Units for pressure, $P$, and temperature, T, in Eq. (4.10) are bars and kelvins, respectively.

Table 4.1. Fitting Parameters for the Liquid/Vapor Saturation Curves.

$\mathrm{C}_{4} \mathrm{H}_{8}$

2826.65

10.5932

$\mathrm{H}_{2} \cdot \mathrm{O}_{2}^{\star}$

716.43

9.2777

*Parameters for $\mathrm{O}_{2}$ and $\mathrm{N}_{2}$ are equal because liquid $n$ itrogen was used as the coolant for the $\mathrm{O}_{2}$ samples.

Liquid nitrogen was also used as a coolant for liquid oxygen samples. The available Hugoniot EOS for liquid oxygen is given for 
initial conditions corresponding to a nitrogen saturation temperature. Hazards involved in combining oxygen with the hydrocarbon oils of diffusion and roughing pumps are avoided by using liquid nitrogen-cooled, gas absorbing molecular sieves, when flushing the specimen cavity.

The pressure measurement of 1-butene for the thermocouple calibration, discussed in Section 4.5, was performed for a sample isolated from the environment. For oxygen and nitrogen samples, the pressure of the cooled cavities is isobaric to atmosphere and it is assumed that the sample is isothermal to the coolant.

Densities for the benzene sample are determined from tabulated values for nominal temperatures at $300 k$ and pressures of 1 bar. Liquid densities for the cryogenic samples are determined by linearly interpolating between two proximate temperature/density values found in Ref. 75-77. These values are given in Table 4.2.

The volume of the condensed sample is calculated from the known reservoir volume, the volume of the transferred gas determined from the reservoir pressures at the start and completion of condensation, and the ratio of the gas to liquid sample densities at the starting temperature and at saturation temperature during condensation. Gauge 1 (Fig. 4.16) begins to show little change in pressure as the volume condensed approaches the sample chamber volume. Condensation is complete once these two values agree. 
Table 4.2. Initial Sample Densities. ${ }^{75-77}$

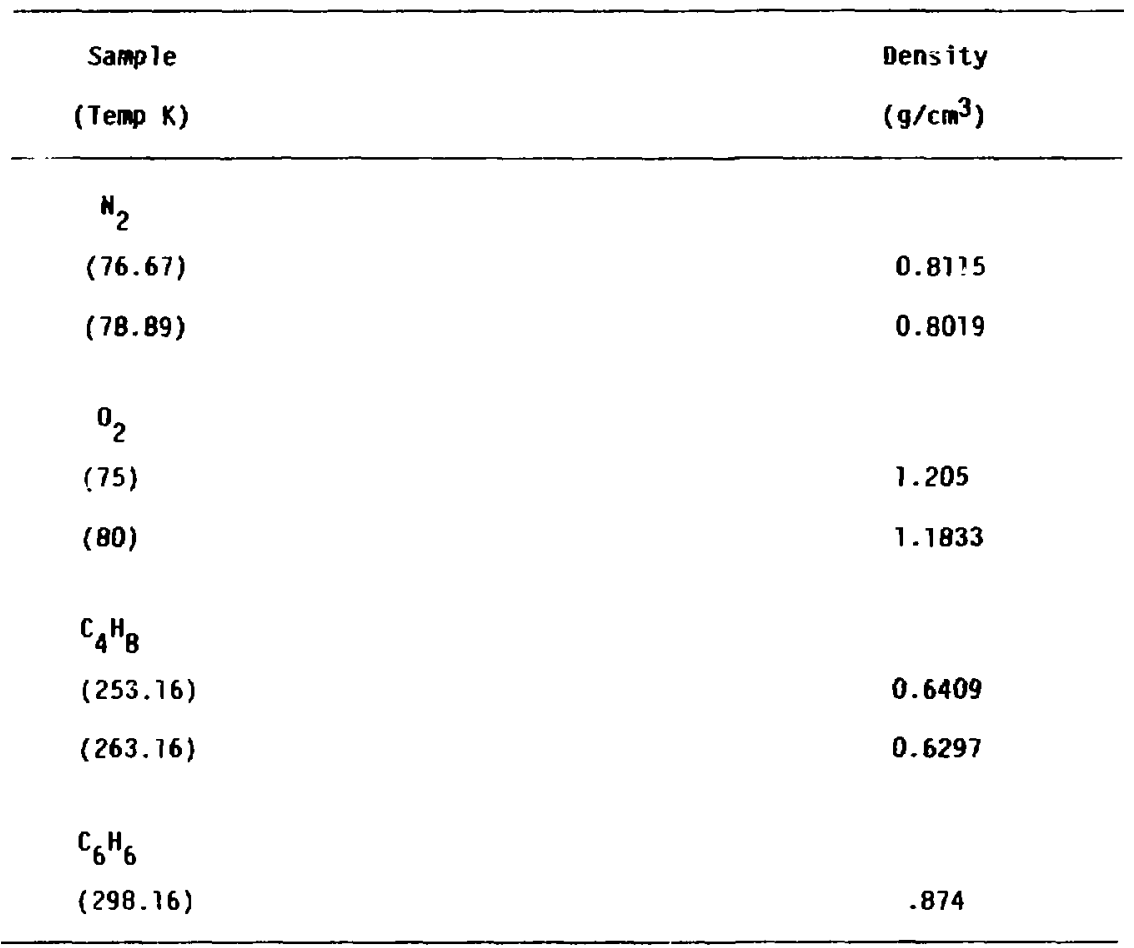

\subsection{Temperature Control.}

A temperature control system is described which can cool and maintain a temperature setting with a variation of less than $0.1 \mathrm{~K} u$ sing the flow of cold nitrogen gas. Strict control is necessary for samples which have a narrow temperature span in the liquid region (for example, xenon). In the case of liquid 1-butene, however, a temperature is desired corresponding to a value just below vapor/liquid saturation 
temperature at atmospheric pressure. The temperature should be low enough to stay safely in the liquid-phase region, but high enough to still benefit from the large density difference between 1-butene and polybutene. A suitable value of $263 \mathrm{~K}$ was chosen and requirad a cooling system depicted in Fig. 4.17. Central to the control system is a logic device which compares a digitized thermocouple output signal to a chosen set-point corresponding to the desired temperature.

A heater coil, immersed in a dewar filled with liquid nitrogen, pressurized the dewar with cold nitrogen gas which flowed through a pneumatic valve, then around the target coolant cavity, and exhausted to the atmosphere. The decision to open or close a pneumatic valve, driven

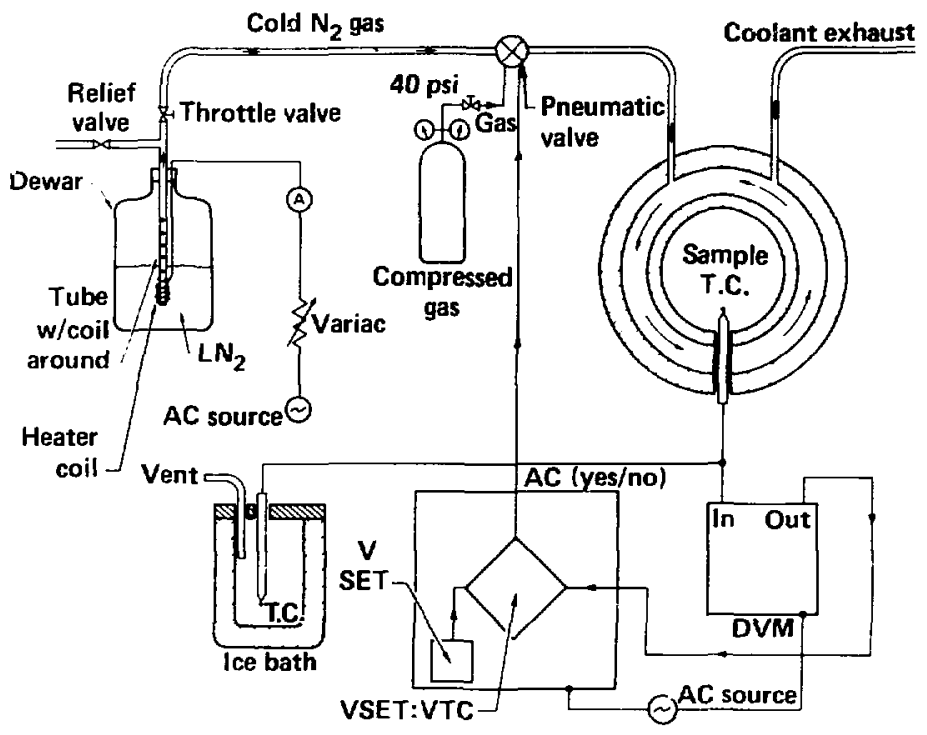

Fig. 4.17 Temperature control system used for liquid 7 -butene samples. 
by a bottle of compressed gas, was based on the difference of the two temperatures which were made available to the comparator.

Calibration of the thermocouples was achieved by slowly approaching the temperature on the 1-butene liquid/vapor saturation curve, from both above and below, until a consistent pair of values in sample cavity pressure and thermocouple readings was found. The final temperature chosen for the liquid sample was usually $1-2 \mathrm{~K}$ below the saturation curve.

\subsection{Mass Velocities and Impedance Matching}

Standard impedance matching techniques are used to obtain the mass velocity. The impactor velocity is determined to an accuracy on the order of $1 x$ and is combined with material $u_{p}-u_{s}$ relations (given in Table 4.3) and densities to determine the final sample properties.

Consider Fig. 4.18 showing the Hugoniot of the impactor (Region I), baseplate (Region II) and sample (Region III) on a $P-U_{p} p l o t$. This space is convenient due to the equalities of pressure and particle velocities at interfaces between two regions.

First the intersection of the Hugoniots for the impactor/baseplates contact is determined. The Hugoniot for the cold aluminum (cryogenic sample) baseplate is represented by curve $A$ and is

$$
P_{B}=P_{B} U_{P B}\left(C_{B}+S_{B} U_{P B}\right) \text {, }
$$

where $\rho_{B}, C_{B}$ and $S_{B}$ is the density and the linear $U_{P}-U_{S}$ fitting parameters 
Table 4.3. $U_{p}-U_{s}$ Parameters Used in Shock Impedance Analys is. $7,16,66$

\begin{tabular}{lcccccc}
\hline Material & $\mathrm{C}$ & $\mathrm{S}_{1}$ & $\mathrm{~S}_{2}$ & $\mathrm{~A}_{0}$ & $\mathrm{~A}_{1}$ & $\mathrm{~A}_{2}$ \\
$($ Temp K) & $(\mathrm{cm} / \mu \mathrm{S})$ & & $\left(\frac{\mu \mathrm{S}}{\mathrm{cm}}\right)$ & & $\left(\frac{\mu \mathrm{S}}{\mathrm{cm}}\right)$ & $\left(\frac{\mu \mathrm{S}}{\mathrm{cm}}\right)^{2}$ \\
\hline $\mathrm{Al}$ & 0.5386 & 1.339 & - & 0.0039 & -0.01082 & 0.03351
\end{tabular}

(300)

$\begin{array}{lllllll}\text { A1 } & 0.5420 & 1.334 & - & 0.0039 & -0.01082 & 0.03351\end{array}$

(80)

$\begin{array}{lllllll}\text { Ta } & 0.3293 & 1.207 & - & 0.00547 & -6.03798 & 0.10209\end{array}$

(300)

$\begin{array}{lllllll}\mathrm{C}_{6} \mathrm{H}_{6} & 0.1721 & 1.330 & - & 0.18023 & -0.5797 & 0.048816\end{array}$

(300)

$\begin{array}{lllllll}\mathrm{N}_{2} & 0.07672 & 1.896 & -0.7931 & 0.0090 \mathrm{~B} & 0.00568 & 0 .\end{array}$

(80)

$\begin{array}{lllllll}0_{2} & 0.2327 & 1.215 & - & 0.020186 & -0.074346 & 0.090296\end{array}$

(80)

1ur the baseplate. Units of pressure are in Mbar when the density and velocities are expressed as $\mathrm{g} / \mathrm{cm}^{3}$ and $\mathrm{cm} / \mu \mathrm{s}$, respectively. The Hugoniot representing the shocked state of the impactor is referenced on the $U_{p}$-axis to its velocity, $U_{1}$, and is reflected about the $P$-axis thus representing a shock traveling to the left and is 


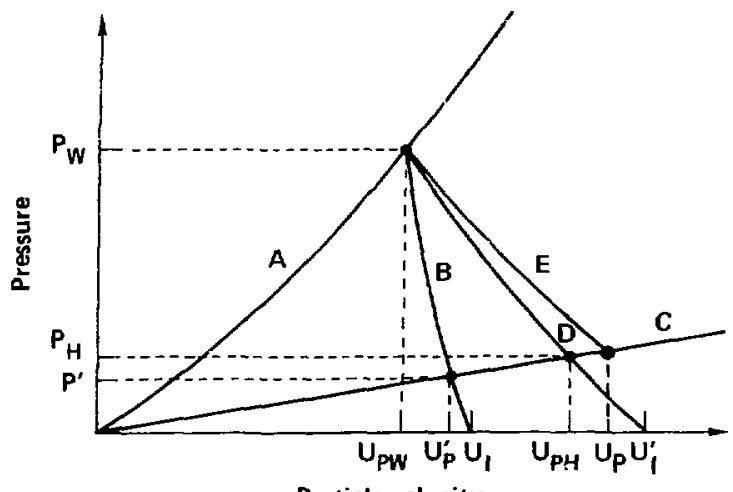

Particle velocity

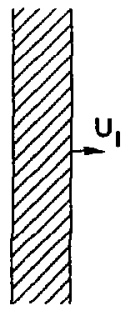

I

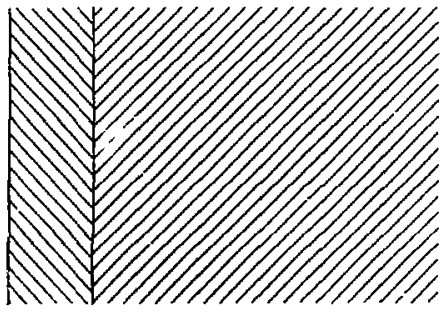

III

Fig. 4.18 Impedance matching considerations in an EOS experiment for determining final thermodynamic state of sample (Region lil, curve C) from impactor (Region I, curve B) and baseplate (Region II, curves $A, D$ and release curve $E$ ! using a $P-U_{p}$ diagram.

$$
P_{I}=p_{I}\left(U_{I}-U_{P I}\right)\left[C_{I}+S_{I}\left(U_{I}-U_{P I}\right)\right],
$$

where $P_{I}, C_{I}$ and $S_{I}$ correspond to the impactor properties.

Due to the equalities $U_{P I}=U_{P B}$ and $P_{I}=P_{B}$, and setting $\beta_{1}=p_{I} / \rho_{B}$, a quadratic expression for $U_{p W}$ is obtained 


$$
\begin{aligned}
\left(S_{B}-\beta_{1} S_{I}\right) u_{p W}^{2} & +\left[C_{B}+\beta_{7}\left(2 S_{I} U_{I}+C_{I}\right)\right] u_{p W} \\
& -\left[\beta_{1} u_{I}\left(S_{I} u_{I}+C_{I}\right)\right]=0,
\end{aligned}
$$

which is the particle velocity of the shocked state of the impactor and baseplate. This new material velocity acquired by the baseplate is equivalent to the baseplate traveling to the right (as a projectile) at an "effective" impactor velocity of $U_{I}$ " $=2 U_{\mathrm{pW}}$. A shock wave continues into the sample once the shock, traveling in the baseplate, reaches the interface. This is due to the lower impedance of the sample compared to the baseplate. For the present, the state of the sample will be described as if the Hugoniot and release isentrope were one and the same (curves $D$ and $E$ ). A correction for this discrepancy will then follow.

The intersection of the Hugoniot of the baseplate (reflected about the vertical axis through $v_{p w}$ ) with the Hugoniot of the sampie is found with the same stipulation as before (that is, $U_{P B}=U_{P S} \equiv U_{p H}$ and $P_{B}=$ $\left.P_{S} \equiv P_{H}\right)$. The baseplate Hugoniot,

$$
P_{B}=\rho_{B}\left(U_{I}^{\prime}-U_{P B}\right)\left[C_{B}+S_{B}\left(U_{I}^{\prime}-U_{P B}\right)\right]
$$

is combined with the sample Hugoniot (curve C)

$$
P_{S}=\rho_{S} U_{P S} U_{S} \text {, }
$$

to obtain an expression for the Hugoniot-reflected particle velocity. $\mathrm{u}_{\mathrm{pH}}$,

$\beta_{2} S_{B} U_{p H}^{2}-\left[U_{S}+\beta_{2}\left(C_{B}+2 S_{B} U_{I}^{\prime}\right)\right] U_{p H}+\beta_{2} U_{I}^{\prime}\left(C_{B}+S_{B} U_{I}^{\prime}\right)=0$, 
where $\beta_{2} \equiv \frac{\rho_{B}}{\rho_{S}}$.

The $U_{p}-U_{S}$ fit for $N_{2}$ is quadratic (instead of lifiear) and requires further manipulation in order to obtain the fina? thermodynamic state. The Hugoniot for nitrogen is given by

$$
u_{S}=c_{N}+s_{1} u_{p}+s_{2} u_{p}^{2}
$$

where the fitting parameters are given in Table 4.1. If this expression is substituted into the expression defining the equivalence of particle velocities and pressures, a new expression cubic in the particle velocity is obtained in the form

$$
\begin{gathered}
f\left(U_{p H}\right) \equiv S_{2} U_{p H}^{3}+\left(S_{1}-\beta_{2} S_{B}\right) U_{p H}^{2}+\left[C_{N}+\beta_{2}\left(2 S_{B} U_{I}+C_{B}\right)\right] U_{p H} \\
-\left[\beta_{2} U_{I}\left(S_{B} U_{I}^{\prime}+c_{B}\right)\right]=0 .
\end{gathered}
$$

This can be solved easily using Newton's method by differentiating $f\left(U_{p H}\right)$ with respect to $U_{p H}$ giving

$$
f^{\prime}\left(U_{p H}\right)=3 S_{2} U_{p H}^{2}+2\left(S_{1}-\beta_{2} S_{B}\right) U_{p H}+\left[C_{H}+\beta_{2}\left(2 S_{B} U_{Z}+C_{B}\right)\right] .
$$

Let $U_{p H} \rightarrow X$ and denote the $n^{\text {th }}$ iteration of $x$ by $x_{n}$. Then NewtonRaphson iterations proceed by solving

$$
x_{n+1}=x_{n}-\frac{f\left(x_{n}\right)}{f^{\prime}\left(x_{n}\right)}
$$

and usually converge to four decimal places within four iterations.

The isentrope-Hugoniot discrepancy for determining the mass velocity is given by 


$$
\frac{u_{p}-U_{p H}}{u_{p H}}=B_{0}+B_{1}\left(U_{p H}-U_{p w}\right)+B_{2}\left(U_{p H}-U_{p w}\right)^{2} \text {, }
$$

where $U_{p}$ is the corrected particle velocity using the wall release isentrope, $B_{0}=.0 .001096, B_{1}=0.05671 \frac{\mathrm{\mu S}}{\mathrm{cm}}$ and $B_{2}=0.1276\left(\frac{\mathrm{\mu s}}{\mathrm{cm}}\right)^{2} .7,66$ The correction is positive, increases with shock pressure and is less than 2\% for the highest dynamic pressures reported here. Equation (4.21) is from Ref. 7, where the $U_{P H}$ in the denominator on the left side of the equation was inadvertently omitted.

\section{B Summary of Experimental Procedure}

- Fabricate target and measurement of pin heights, baseplate and impactor thicknesses and densities, and rel! constants and shunt resistor values (where applicable).

- Set delays for triggering, signal cables and flash $x$-rays.

- Measure cable lengths.

- Adjust scope sensitivities.

- Mount and align targets with set-up flash $x$-ray pictures.

- Pump down target chamber to about $10 \mathrm{\mu m}$.

c Flush sample cavity and begin cooling (if applicable).

- Calibrate thermocouples and prepare sample.

o Take baseline sweep, calibrations and return scopes to firing status (if applicable).

- Take sample of the specimen gas for chemical analysis.

- Record final sample cavity temperature and pressure before gun fire. 
The results of the EOS measurements for liquid 1 -butene $\left(\mathrm{CH}_{2}=\mathrm{CH}-\mathrm{CH}_{2}-\mathrm{CH}_{3}\right)$ and electrical conductivity measurements for liquids benzene $\left(\mathrm{C}_{6} \mathrm{H}_{6}\right)$, nitrogen $\left(\mathrm{N}_{2}\right)$ and oxygen $\left(\mathrm{O}_{2}\right)$ are given in Tables 5.1 and 5.2 and are shown in Figs. 5.1-5.4. The 5 order-of-magnitude change in the electrical conductivity over the dynamic pressure range is conveniently plotted on a semi-logarithmic scale. Detajls of baseplate and impactor densities and dimensions, electrode and pin positions, cell constants and electrode materials for each target are given in Appendix 0. EOSi data for liquid 1-butene display a linear character on a $U_{p}-U_{s} p l o t$ over the pressure range $12.3-53.8 \mathrm{GPa}$.

A determination of the uncertainties in the shock pressure is based on random experimental error, systematic experimental error and systematic bias in the data analysis and is described in Ref, 66. The experimental error in mass velority is determined by computing the

Table 5.7. Hugoniot data for liquid 1-butene $\left(\mathrm{CH}_{2}=\mathrm{CH}-\mathrm{CH}_{2}-\mathrm{CH}_{3}\right)$ obtained with a two-stage light-gas gun.

\begin{tabular}{|c|c|c|c|c|c|c|c|c|}
\hline Shot. & Impacto & $r \mathrm{U}_{\mathrm{I}}$ & $T_{0}$ & $P_{0}$ & $u_{p}$ & $v_{s}$ & $\mathbf{P}$ & $v$ \\
\hline & & $\left(\frac{\mathrm{Km}}{\mathrm{s}}\right)$ & (K) & $\left(\frac{\mathrm{g}}{\mathrm{cm}^{3}}\right)$ & $\left(\frac{\mathrm{Km}}{\mathrm{s}}\right)$ & $\left(\frac{k m}{s}\right)$ & (Kbar) & $\left(\frac{c m^{3}}{m o i e}\right)$ \\
\hline LCAHBEQ1B & Al & 5.425 & 262.6 & 0.630 & $4.35 \pm 0.03$ & $7.66 \pm 0.08$ & $210 \pm 3$ & $38.4 \pm 0.7$ \\
\hline LC4HBEQ2 & $\mathrm{Ta}$ & $6.46 ?$ & 262.7 & 0.630 & $7.43 \pm 0.05$ & $11.48 \pm 0.17$ & $538 \pm 9$ & $31.4 \pm 0.9$ \\
\hline LCAHBEQ4 & Al & 3.907 & 262.6 & 0.630 & $3.22 \pm 0.02$ & $6.07 \pm 0.06$ & $123 \pm 2$ & $41.7 \pm 0.7$ \\
\hline
\end{tabular}


Table 5.2. Electriral conductivities of shock-compressed benzene $(C B Z-\ldots)$, nitrogen $(N C-\ldots)$, and oxygen $(L 02-\ldots)$.

$\begin{array}{ccccccc}\text { Shot Impactor } & U_{I} & T_{0} & P_{0} & V / V_{0} & P & \sigma \\ & (\mathrm{Km} / \mathrm{s}) & (\mathrm{K}) & \left(\mathrm{g} / \mathrm{cm}^{3}\right) & & (\mathrm{GPa}) & \text { (ohm }^{-1}\left(\mathrm{cmll}^{-1} \text {; }\right.\end{array}$

\begin{tabular}{llllllll}
\hline CBZ-4 & A1 & 6.801 & 298 & 0.874 & $0.401 \pm 0.005$ & $37.8 \pm 1.8$ & $49 \pm 27$ \\
CBZ-5 & Al & 6.112 & $29 B$ & 0.874 & $0.413 \pm 0.004$ & $31.7 \pm 1.3$ & $2.4 \pm 0.4$ \\
CBI-7 & A1 & 4.798 & 298 & 0.874 & $0.443 \pm 0.003$ & $21.4 \pm 0.5$ & $2.3 \times 10^{-3} \pm 0.2 \times 10^{-3}$ \\
CBZ-10 & A1 & 7.073 & 298 & 0.874 & $0.396 \pm 0.005$ & $40.4 \pm 2.0$ & $30 \pm 6$ \\
CBZ-11 & A1 & 7.083 & 298 & 0.874 & $0.396 \pm 0.003$ & $40.5 \pm 1.9$ & $20 \pm 3$ \\
CBZ -14 & A1 & 5.505 & 298 & 0.874 & $0.425 \pm 0.004$ & $26.8 \pm 0.9$ & $0.05 \pm(.02 .0 .2)$ \\
CBZ -15 & Ta & 5.405 & 299 & 0.873 & $0.382 \pm 0.003$ & $50.1 \pm 2.7$ & $46 \pm 9$ \\
CBZ 16 & Ta & 0.119 & 301 & 0.870 & $0.370 \pm 0.004$ & $61.4 \pm 3.7$ & $36 \pm 5$
\end{tabular}

$\begin{array}{llllllll}\mathrm{NC}-3 & \text { Al } & 7.574 & 71.4 & 0.808 & 0.366 \pm 0.032 & 41.5 \pm 0.5 & 8 \pm 1\end{array}$

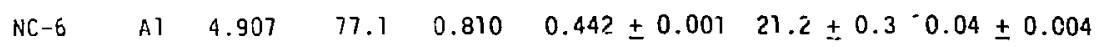

$\begin{array}{llllllll}N C-g & \text { Al } & 6.586 & 77.3 & 0.809 & 0.394 \pm 0.002 & 33.8 \pm 0.4 & 2.4 \pm 0.3\end{array}$

$\begin{array}{lllllll}N C-9 & \text { Al } & 5.660 & 77.2 & 0.809 & 0.420 \pm 0.001 & 26.6 \pm 0.4 \cdot 0.1 \pm 0.02\end{array}$

NC-10 Al $4.626 \quad 77.6 \quad 0.807 \quad 0.444 \pm 0.007 \quad 19.1 \pm 0.3 \quad 1.5 \times 10^{-3} \pm 0.2 \times 10^{-3}$

$\begin{array}{lllllll}\text { NC-11 Ta } & 6.539 & 76.2 & 0.813 & 0.297 \pm 0.003 & 61.6 \pm 0.9 & 47 \pm 6\end{array}$

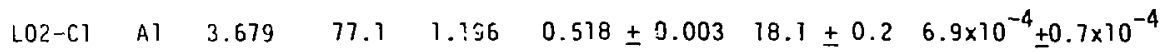

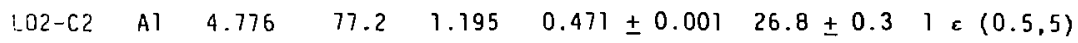

$\begin{array}{llllllll}\text { LO2-C3 Ai } & 5.464 & 77.1 & 1.196 & 0.448 \pm 0.001 & 33.0 \pm 0.4 & 28 \pm 10\end{array}$

$\begin{array}{llllllll}02-C 5 & \text { A1 } & 4.345 & 76.5 & 1.199 & 0.488 \pm 0.002 & 23.3 \pm 0.3 & 0.09 \pm 0.02\end{array}$

$\begin{array}{lllllll}102-[6 \quad 41 \quad 6.467 & 76.7 & 1.198 & 6.420 \pm 0.001 & 43.0 \pm 0.5 & 73 \pm 10\end{array}$ 


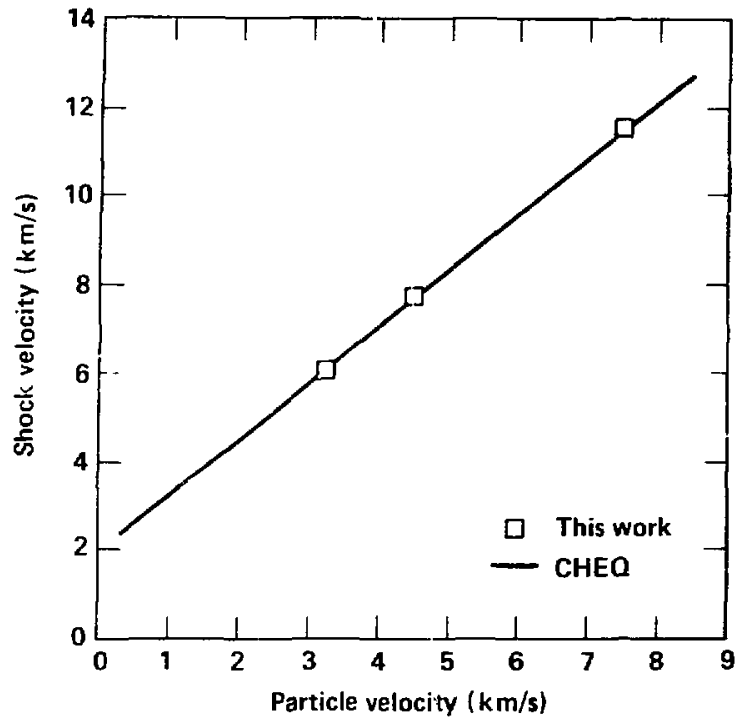

Fig. 5.1 1-butene Hugoriot data in $u_{p}-u_{s}$ space. The full curve indicates CHEQ results using the reactive model.

differences between the maximum and misrimum particle velocities associated with sequentially assigning each experimental variable its highest and lowest possible value, then sumning their root-mean-squares. The systematic error is obtained by constructing bands of uncertainty about each Hugoniot in the $U_{p}-p$ plane and locating the associated extremum intersections. The band width is quadratic in the particle velocity. The total uncertainty is the arithmetic sum of the experimental and systematic error.

The data for the Hugoniot of 1-butene fit a linear relation $U_{s}=$ $0.2024 \mathrm{~cm} / \mu \mathrm{s}+1.274 \mathrm{u}_{\mathrm{p}}$. The curve appearing in $\mathrm{Fig} .5 .1$ is a result 


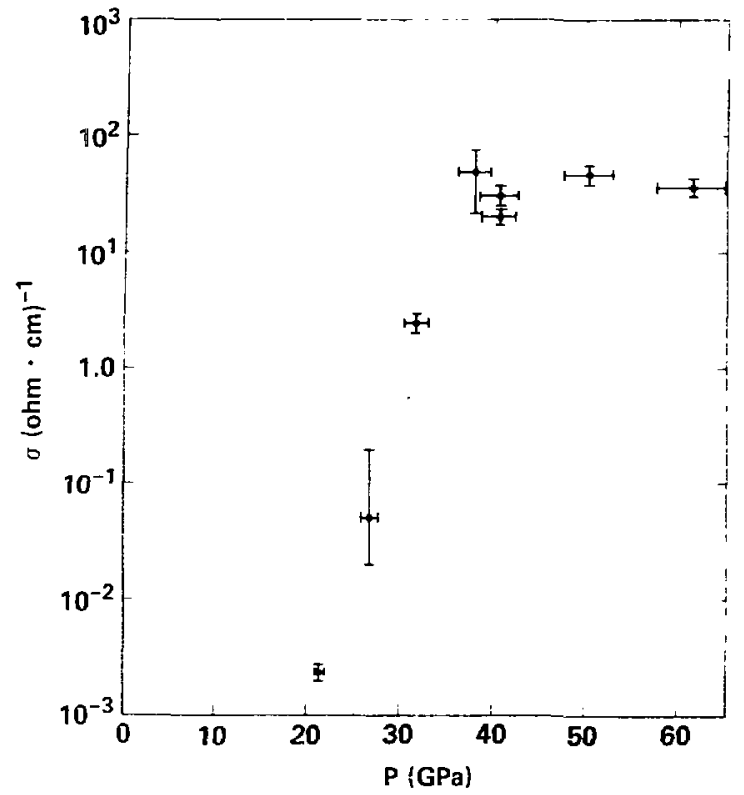

Fig. 5.2 Benzene electrical conductivity data as a function of shock pressure.

of Hugonint calculations from the CHEQ code which assumes a reactive model discussed in the next chapter.

The electrical conductivity results all display an initially steep rise over the first 20-30 GPa then appear to saturate just below 100 $\Omega^{-1} \mathrm{~cm}^{-7}$ at iigher shock pressures. Ross' calculated fractional dissociation, $x_{2}$, appearing in Fig. 5.3, was previously discussed in Chapter 2 and will be reiterated in Section 6.2 , which discusses the results. 


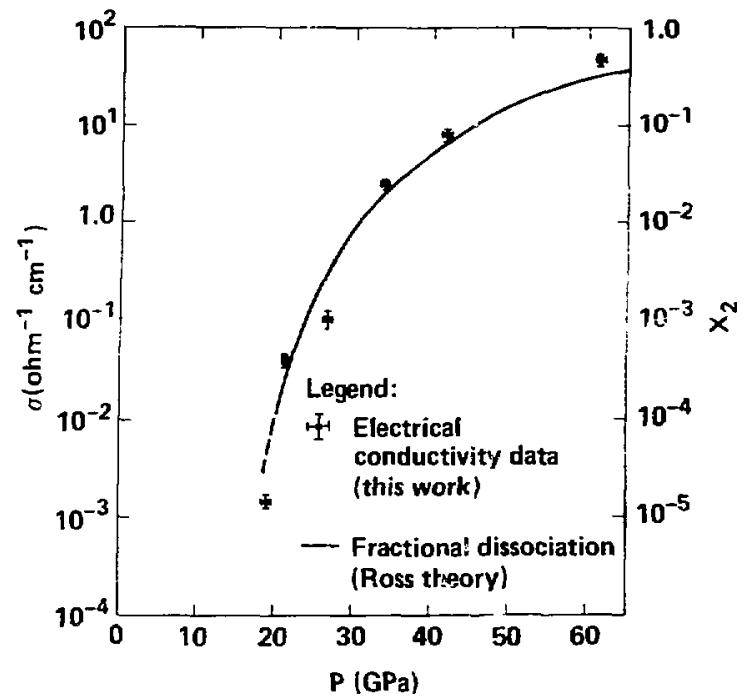

Fig. 5.3 Nitrogen electrical conductivity data (left absissa) and Ross' calculated fractional dissociation (right absissa) as a function of shuck pressure. 


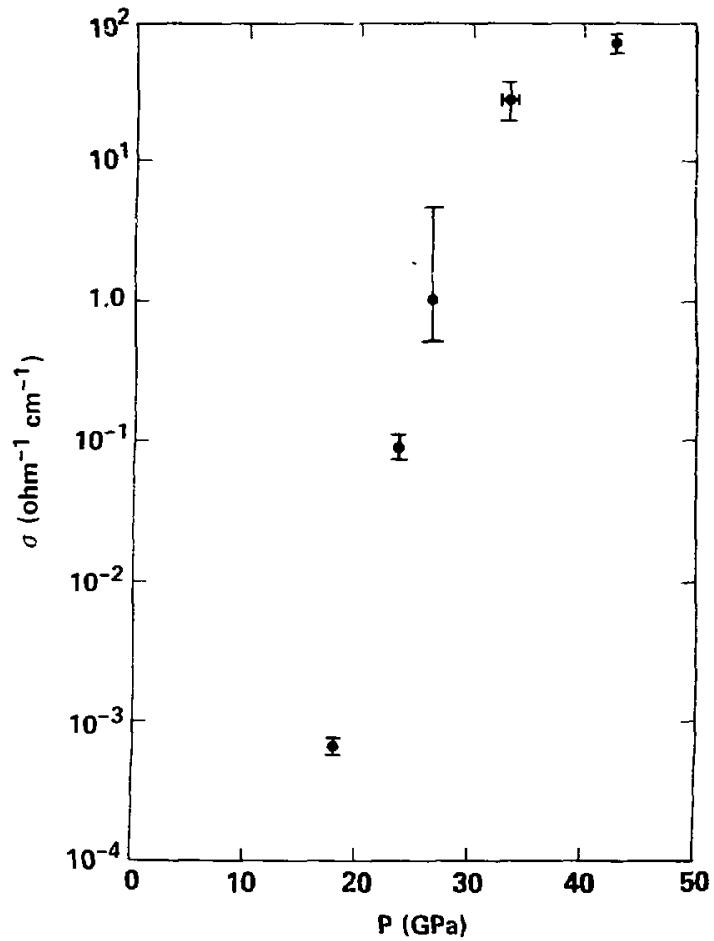

Fig. 5.4 0xygen elertrical conductivity data as a function of shock pressure. 


\subsection{I-Butene $\left(\mathrm{CH}_{2}=\mathrm{CH}-\mathrm{CH}_{2}-\mathrm{CH}_{3}\right)$ Equation of State}

The curve in Fig. 5.7 corresponds to theoretical results using a statistical mechanical reactive chemical equilibrium code (CHEQ) developed by Ree. ${ }^{3}$ The calculations allow only the carbon (diamond-like phase) and molecular hydrogen phase by forcing a dissociation reaction such as

$$
2 \mathrm{C}+\mathrm{H}_{2} \rightarrow \mathrm{C}_{2} \mathrm{H}_{2}
$$

to the left. This is done by choosing an unrealistically large heat of formation for $\mathrm{C}_{2} \mathrm{H}_{2}$. The calculated Hugoniot agrees with these experimental recults to within the experimental uncertainly $(\sim 1.6 \%)$ over the pressure range $(12.3-53.8 \mathrm{GPa})$ investigated.

As discussed in chapter 2, a Hugoniot point is determined (from CHEQ) by selecting a temperature and finding a set of thermodynamic variables $\{P, T, V(P, T), E(P, T)\}$ which satisfy $E q .(3.37)$. The initial energy appearing in the energy Hugoniot relation is obtained by a procedure outlined in Appendix $B$. The reference state is the gaseous phases of molecular hydrogen and graphite at $298.16 \mathrm{~K}$ and $1 \mathrm{~atm}$.

Calculated temperatures closely correspond to a linear dependence with dynamic pressure and lie between $7400-5550 \mathrm{~K}$ corresponding to a pressure range 12.3-54.8 GPa. The substantial increase in 1-butene's $\left(\mathrm{CH}_{2}=\mathrm{CH}-\mathrm{CH}_{2}-\mathrm{CH}_{3}\right)$ temperature over that of polybutene $\left[\left(\mathrm{CH}_{2}-\mathrm{CH}_{-}-\mathrm{CH}_{2}-\mathrm{CH}_{3}\right)_{n}\right]$, 
which was calculated to lie between $830-3100 K$ for the same dynamic pressure range, ${ }^{16}$ reflects the increasing contribution of the therma 1 component of the pressure for a stoichiometrically equivalent fluid with a smaller initial density and larger shock energy.

A pressure of $33.4 \mathrm{GPa}$ is calculated for a low temperature $(230 \mathrm{~K}$ ) isotherm at a density corresponding to the highest shock compressed state achieved in the experiments $\left(P=53.8 \mathrm{6Pa}, \rho=1.789 \mathrm{~g} / \mathrm{cm}^{3}\right)$. This temperature is low enough to approximate the $0-K$ isotherm adequately and high enough to insure that the specific heat values used in the analysis are reliable. The thermal contribution to the total pressure for 1-butene is $38 \%$, about 2-1/2 times larger than for polybutene compressed to the same final volume.

Extending the calculations to include a progressively larger collection of hydrocarbon species indicates a trend similar to that found in Ref. 16 for benzene and polybutene. The following reactions are considered and are grouped in 4 blocks, each containing an alkane, $\mathrm{C}_{m} \mathrm{H}_{2 m+2}$, of progressively larger order, $m$ : 


$$
\begin{aligned}
\mathrm{C}+2 \mathrm{H}_{2} & =\mathrm{CH}_{4} \\
\mathrm{H}_{2}+2 \mathrm{C} & =\mathrm{C}_{2} \mathrm{H}_{2} \\
\mathrm{C}_{6} \mathrm{H}_{6} & =3 \mathrm{C}_{2} \mathrm{H}_{2} \\
\mathrm{H}_{2}+\mathrm{C}_{2} \mathrm{H}_{2} & =\mathrm{C}_{2} \mathrm{H}_{4} \\
\mathrm{H}_{2}+\mathrm{C}_{2} \mathrm{H}_{4} & =\mathrm{C}_{2} \mathrm{H}_{6} \\
3 \mathrm{C}+3 \mathrm{H}_{2} & =\mathrm{C}_{3} \mathrm{H}_{6} \\
\mathrm{C}_{3} \mathrm{H}_{6}+\mathrm{H}_{2} & =\mathrm{C}_{3} \mathrm{H}_{8} \\
\mathrm{C}_{2} \mathrm{H}_{4}+\mathrm{C}_{2} \mathrm{H}_{6} & =n-\mathrm{C}_{4} \mathrm{H}_{10} \\
\mathrm{C}_{2} \mathrm{H}_{6}+\mathrm{C}_{3} \mathrm{H}_{6} & =n-\mathrm{C}_{5} \mathrm{H}_{12} .
\end{aligned}
$$

\begin{tabular}{|c|c|c|c|c|}
\hline Species & $\begin{array}{c}\text { Group I } \\
m^{\star}=2\end{array}$ & $\begin{array}{c}\text { Group II } \\
m^{\star}=3\end{array}$ & $\begin{array}{c}\text { Group III } \\
m^{\star}=4\end{array}$ & $\begin{array}{l}\text { Group IV } \\
m^{\star}=5\end{array}$ \\
\hline $\begin{array}{l}\text { Diamond } \\
\mathrm{H}_{2} \\
\mathrm{CH}_{4} \\
\mathrm{C}_{2} \mathrm{H}_{2} \\
\mathrm{C}_{6} \mathrm{H}_{6} \\
\mathrm{C}_{2} \mathrm{H}_{4} \\
\mathrm{C}_{2} \mathrm{H}_{6} \\
\mathrm{C}_{3} \mathrm{H}_{6} \\
\mathrm{C}_{3} \mathrm{H}_{8} \\
\mathrm{C}_{4} \mathrm{H}_{10} \\
\mathrm{nC}_{5} \mathrm{H}_{12} \\
\text { A1t hydrocarbons }\end{array}$ & $\begin{array}{l}4.71 \times 10^{-1} \\
8.14 \times 10^{-2} \\
6.74 \times 10^{-2} \\
1.37 \times 10^{-4} \\
5.67 \times 10^{-4} \\
0.00 \\
2.61 \times 10^{-1}\end{array}$ & $\begin{array}{l}3.13 \times 10^{-7} \\
7.31 \times 10^{-2} \\
2.47 \times 10^{-2} \\
6.66 \times 10^{-5} \\
7.09 \times 10^{-5} \\
0.00 \\
4.42 \times 10^{-2} \\
1.99 \times 10^{-2} \\
1.72 \times 10^{-1}\end{array}$ & $\begin{array}{l}2.77 \times 10^{-7} \\
7.66 \times 10^{-2} \\
1.22 \times 10^{-2} \\
4.14 \times 10^{-5} \\
1.58 \times 10^{-5} \\
0.00 \\
1.30 \times 10^{-2} \\
5.25 \times 10^{-3} \\
3.14 \times 10^{-2} \\
1.44 \times 10^{-1} \\
2.05 \times 10^{-1}\end{array}$ & $\begin{array}{l}2.47 \times 10^{-1} \\
7.91 \times 10^{-2} \\
5.65 \times 10^{-3} \\
2.51 \times 10^{-5} \\
2.92 \times 10^{-6} \\
0.00 \\
5.67 \times 10^{-3} \\
1.18 \times 10^{-3} \\
4.55 \times 10^{-3} \\
4.73 \times 10^{-2} \\
1.17 \times 10^{-1} \\
1.69 \times 10^{-1}\end{array}$ \\
\hline
\end{tabular}

(1I)

(IV) (6.2)

Table 6.1. Calculated dissociation products of 1-butene (in moles) per mole of $\cdot \mathrm{CH}_{2}$ at $\mathrm{T}=5800 \mathrm{~K}$ and $\mathrm{P}=56.0 \mathrm{GPa}$.

The equilibricm concentrations calculated for groups I-IV, corresponding to the largest alkane $m=2-5$, are given in Table 6.1 . The values given in the last row are the sum of all hydrocarbons and decrease for 
increasing $m$. This suggests that inciuding larger species in the reaction list could eventually reduce the product to two species, diamond and molecular hydrogen.

The excellent agreement between experimental data and a model assuming a two-phase mixture for the entire dynamic pressure range investigated supports Ree's model that 1-butene is completely dissociated for dynamic pressures above $12.3 \mathrm{GPa}$.

\subsection{Nitrogen Electrical Conductivity}

Here, a model for the observed electrical conductivity of nitrogen is described. Two assumptions are made: (1) Ross' model, for the shockcompressed state of nitrogen, is valid. (2) The monatomic component of the $N_{2}-\mathrm{N}$ mixture will exhibit bonding properties typical of other elements of the Group $V$ column of the periodic table and will be the component primarily responsible for the measured finite conductivities. A comparison is drawn between the monatomic form of nitrogen and electrical conductivity measurements performed on hot, dense metallic vapors.

As was stated in Chapter 2, good agreement with Hugoniot data was achieved when Ross allowed for molecular dissociation. ${ }^{13}$ The reaction

$$
\mathrm{N}_{2} \rightarrow 2 \mathrm{~N}
$$

occurs over the entire dynamic pressure range in which electrical conductivity measurements were made, but becomes significant for 
pressures in excess of $30 \mathrm{GPa}$. Dissociation energies for reaction (6.3) are density dependent, with the effect of reducing the initial value of $9.8 \mathrm{eV}$ (for a free molecule) to values comparable to the temperatures accompanying the shocked state $(\sim 1 \mathrm{eV})$. Russ' calculations provide two important quantities: the temperature and the dissociation fraction, $x_{2}$. The calculated dissociation fraction changes from $10^{-4}$ to nearly 0.5 in the pressure range $20-70 \mathrm{GPa}$ and is indicated by the solid curve in Fig. 5.3 .

For a nontrivial extent of reaction, one must consider both species of nitrogen described by (6.3): one is a rather loosely packed collection of electrically, inert species interacting by short-range van der waaltype forces. This is diatomic nitrogen. The other is monatomic nitrogen and is expected to exhibit bonding properties typical of other semimetals in the Group $V$ column like phosphorous and arsenic. One of the more distinctive features of the metallic form is an increased coordination number between other monatomic neighbors which will tend to increase the packing efficiency. The prospect of a random, relatively dilute collection of atoms, with metallic-like bonding properties, mixed into a comparatively inert medium prompts a comparison with twa ather similar phenomena: doped semiconductors and metallic vapors. Though all three are disordered, the higher temperatures characteristic of these experiments suggest a comparison with metallic vapors.

The remainder of this section will include a review of some experimental results on electrical conductivity measurements on hot, dense metallic vapors, the interpretation of these results in terms of 
models which successfully describe electronic transport in disordered materials, and finally how these apply to the nitrogen results.

Much work on the measurement of transport properties of metallic vapors, was performed to determine their applicability to MHD problems. Hoshino and coworkers measured the electrical conductivity of fluid selenium over a wide temperature and pressure range (some of which were supercritical). ${ }^{78}$ Renkert, Hensel and Frank reported electrical conductivity results for dense cesium vapor. ${ }^{79}$ Hensel measured the optical absorption of dense mercury vapor. ${ }^{80}$ Generally, the transport. properties were measured along an isotherm and/or isobar, and revealed that a iransformation to a nearly-metallic state could be achieved, either by increasing the temperature while in the liquid range or increasing the density while in the vapor range. Two basic transport regimes were recognized and depended on the vapor density. For low densities, electronic transport was either by thermally-activated hopping, which displayed an exponential dependence with respect to interatomic separation, or thermal excitation of carriers across a mobility gap which was characterized by ah Arrhenius dependence on temperature. In the low density case, a plot of $\log \sigma$ as a function of 1/T displayed a fairly constant activation energy, until a maximum conductivity was reached $\left(\sigma \sim 100-1000 \Omega^{-1} \mathrm{~cm}^{-1}\right)$, where there was a saturation and eventual decline. The fall-off in o was attributable to an increase in interatomic separation as the temperature increased. The activation energy,

$$
\Delta E=\lim _{\Delta T \rightarrow 0}\left|\frac{\Delta \ln \sigma}{\Delta \frac{T}{T}}\right|,
$$


depended on the density of the vapor and would decrease to zero as the density approached a critical value signifying a metallic regime (Mott transition).

Mott is responsible for a large part of the theoretical work which describes electronic processes in dense, hot metallic vapors. A brief description of Mott's model, which is covered extensively in Refs. 81-83, follows.

Mott considers the responses of a collection of single valence atoms occupying lattice positions while changing the lattice constant. At low densities the atoms are described by localized atomic orbitals which place an electron on each atom. The wave function extends over only one interatomic separation. Any electronic transfer from one atom to the next requires the accommodation by electrons on the receiving atom, and results in a correlation energy. For low densities the energy necessary to make the transfer is on the order of $E \sim 1-A$, where $I$ and $A$ are the ionization energies and electron affinities, respectively. As the atoms are brought closer together the atomic wave functions begin to overlap which can lead to binding and finite bandwidths. The bandwidth has a roughly exponential dependence on separation and results in delocalization of the wave function. Delocalized or extended means that the magnitude of the electron wave function is of the same order everywhere in the crystal. The two competing effects, localization, typical of insulators $(\sigma \rightarrow 0$ as $T \rightarrow 0)$, and delocalization, which includes many-electron transfer effects, became comparable when the energy spread, for an electron resonating between an atom and its nearest neighbor, 
becomes comparable to the bandwidth at a particular energy level. 42 Near the transition the Coulomb potential decreases in its ability to allow for bound states and this is due to the screening effects of the valence electrons. At a critical electron density, corresponding to, a critical atomic density, the screening length, $\lambda$, becomes comparable to the Bohr radius, $a_{0}$, and delocalization occurs.

The electrical conductivity of a material at finite temperature can be written,

$$
\sigma=e \int g(E) \mu(E) f(E)[1-f(E)] d E
$$

where $\mu(E), g(E)$, and $f(E)$ are the mobility, density of states, and Fermi-oirac function at energy $E$, respectively. The mobility is the magnitude of the drift velocity per unit electric field and is in units of $\mathrm{cm}^{2} \cdot v o l t^{-1} \cdot \mathrm{sec}^{-1}$. The density of states, $\mathrm{g}(E)$, is defined as the number of energy states per unit volume per unit energy and is calculated by solving the Schrödinger equation for the appropriate potential energy and boundary conditions of the material. The periodicity of crystalline structures simplifies the mathematical treatment involved in calculating the electronic properties. The strategy of ten used makes use of the fact that the one-electron potential energy must exhibit the periodicity of the lattice. Group theory arguments reduce the problem to one of solving for the primitive cell from which the whole lattice may be generated. Electron states are conveniently written as Bloch waves which extend throughout the crystal and represent delocalized, current-carrying species. Because of the periodicity, the density of states takes the form of alternating regions of energy with large densities, called bands, 
separated by regions where no states are allawed, called gaps. Figure 6. 1a is a schematic representation for the density of states of a crystalline material. The Fermi energy, which is the energy such that the occupational probability equals $1 / 2\left(f\left(E_{F}\right)=1 / 2\right)$, is shown at midgap. The delocalized electron waves Bragg reflect from lattice planes and there exisi certain energies where destructive interference occurs. The position of these energies, two of which form the upper valence band
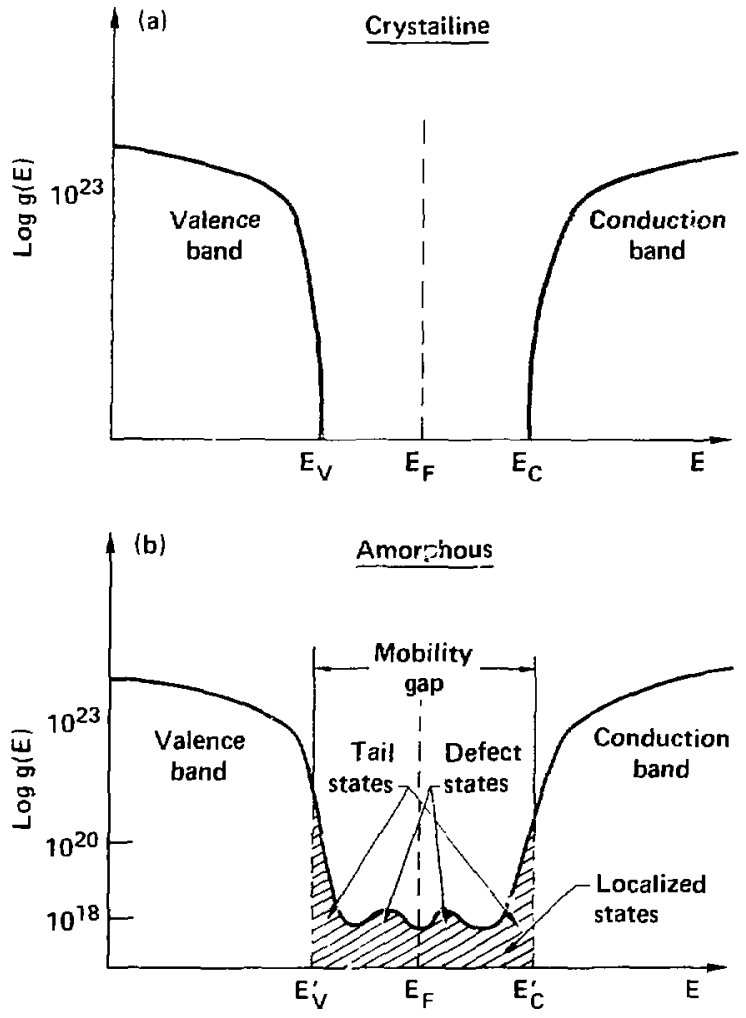

Fig. 6.1 Comparison of the density of states for (a) crystalline and (b) amorphous semiconductors (Ref, 81). 
edge, $E_{v}$, and lower conduction band edge, $E_{c}$, are called van Hove singularities. The approach taken for determining the electronic transport properties of disordered materials differs from that followed for crystalline materials. The lack of long-range order necessitates the approach for determining electronic properties for amorphous materials without reference to any periodic lattice. No reciprocal lattice space can be constructed so that Bloch states cannot be used. If periodicity were essential to observed electrical properties of materials, a large change would be expected upon melting. However, it is observed for a large class of materials, including insulators, semicondurtors, and metals, that only a gradual transition exists in the electrical properties on either side of the melting line. ${ }^{82}$ It follows that not oniy must the density of states be relatively independent of long-range order, but so aiso must be the transport properties such as the mobility. Gaps shou?d exist in the density of states and are apparent in glass, which has a gap width of about $10 \mathrm{eV}$. If short-range inceractions dominate, then it is the short-range order which will determine such properties as the density of states. The basic properties which oistinguish amorphous semiconductors froi: crystalline semiconductors, particularly the Group $1 V$-type, are as follows.

1. Amorphous materials also have bands of electron energy states which extend throughout the material. Delocalized electrons propagate in these states as nearly free charge carriers. Electron drift mobilities for crystaliine materials can exceed $10^{4} \mathrm{~cm}^{2} / v o l t \cdot s e c$ which is equivalent to mean free paths on the order of $10^{3}$ lattice constants. Much shorter meanfree-paths ( -10 lattice constants) are typical for electron transport 
in amorphous materials. In either case, Cohen sets the lower limit for the mobility $(T=300 \mathrm{~K})$ in extended state conduction at $10 \mathrm{~cm}^{2} / \mathrm{volt} \cdot \mathrm{sec} .{ }^{4 B}$

2. Disorder in the lattice structure also manifests itself in the electron density of states by replacing the sharp van Hove singularities at $E_{c}$ and $E_{v}$, which are characteristic of crystalline materials, with a more gradual decreasing density of states extending into the gap (Fig. 6.1b). These electron states, called tail states, are due to deviations of bond angles and bond lengths from their equilibrium values. A tight binding approximation, for determining the density of states, revea is the existence of these states. ${ }^{87,82}$ Tail widths are generally on the order of $0.1 \mathrm{eV}$ for vapor-deposited siticon and increase with smperature. The states in this region (tail states) are localized and therefore do not contribute to carrier transport at low temperatures. The energies in the valence and conduction bands, separating localized from extended states, are denoted by $E_{v}^{\prime}$ and $E_{c}^{\prime}$, respectively and are called mobility edges. At nigher temperatures, the tail states can be emptied and become trapping sites for carriers near the mobility edges. The tail region density of states is typically $10^{-3}$ that of the band states.

3. Deeper in the gap, there are local states originating from atoms with atypical valency. Under-coordination (for example, $z=3$ ) corresponds to a dangling bond. Tight-binding calculations indicate that dangling bonds yield two lacalized states in the gap; one filled and one empty. ${ }^{81, B 2}$ The dangling bond is a non-bonding orbital, contains a single electron, and will therefore have an energy at the zero-energy between the bonding and antibonding levels (the precise position of which 
depends on the details of structural relaxation in the vicinity of the defect). Another possibility, observed in Raman experiments, is two-fold coordinated carbon atoms. The density of these defect states is typically between $10^{-5}$ and $10^{-7}$ of the band-state densities.

4. The extended states in the highest filled band ivalence) are separated from the extended states in the lowest empty band (conduction) by a mobility gap. Localized states exist throughout the energy region between these extended states. Transport of carriers in these localized states is by thermally-activated hopping between states. Mobilities are several orders of magnitude lower than extended state mobilities. For this reason the term "forbidden gap," used for crystalline materials, is replaced with the term "mobility gap," when discussing amorphous materials. The shaded region in Fig. 6.1b, bounded by the mobility edges $E_{v}^{\prime}$ and $E_{c}^{\prime}$, represents the localized states.

In view of the absence of a rigorous theoretical treatment of hot dense vapors, it seems a reasonable next step to extend the results of the previous section, concerning the effects of interatomic separation and disorder on the density of states, to hot metallic vapors whose density is greater than that of the amorphous materials discussed in Mott and Davis. 81 This is a big step.

Figure $6.2 a$ depicts a. density regime that is large enough to cause band overlap. The gap is replaced by a minimum in the density of states located near $E_{F}$. As the interatomic separation increases, the minimum in the density of states near $E_{F}$ decreases until a delocalized region 


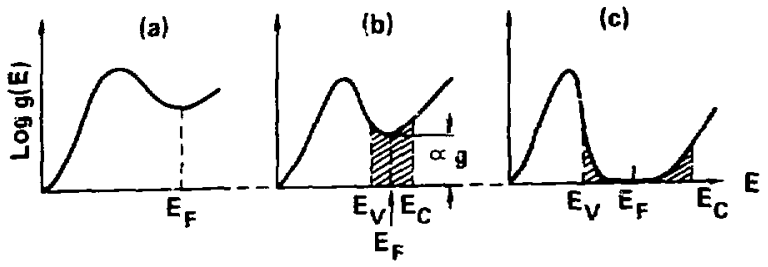

Fig. 6.2 The formation of a pseudogap in the density of states for increasing interatomic separations.

is formed (shaded region of Fig. 6.2b). The ratio of the density of states for the free electrons to the minimum, as a gap is formed, is defined as

$$
g=\frac{g\left(E_{F}\right)}{g\left(E_{F}\right)_{\text {Free }}}
$$

Experimental and theoretical determinations of minimum $g$ for delocalization gives a range for $g$ between 0.2 and 0.3 . At localization $(g-0.2)$, the electron mean-free-path is on the order of a lattice spacing. A crude estimate for a minimum metallic conductivity can be obtained by using the Drude expression

$$
\sigma_{\min } \sim \frac{S_{F} e^{2} a}{12 \pi^{3} h} g^{2} .
$$

together with the minimum condition $g \sim 0.2$. An estimace of the area of the Fermi surface $S_{F}$, is given by

$$
S_{F}=4 \pi K_{F}^{2}=4 \pi\left(\frac{2 \pi}{a}\right)^{2}
$$

As the density decreases, g will decrease below 0.2 and a new 
transport regime entered; that of themally-activated hopping from an occupied to an unoccupied site. In this case the electron drift mobility, $\mu$, is related to the probability, $P$, of making a jump by

$$
\boldsymbol{\mu}=\frac{e \mathrm{a}^{2}}{\mathrm{KT}} \mathbf{p}
$$

The jump probability is proportional to the product of three functions,

$$
p \sim a^{2}(T) e^{-E_{b} / K T} J^{2}(a)
$$

where $a$ and $E_{b}$ are the hopping distance and binding energy, respectively. The wave function overlap, $J$, between adjacent atoms which is expressed as

$$
J \sim e^{-a / \lambda},
$$

reflects the strong dependence of hopping-type transport on interatomic separation.

A further reduction in fluid density decreases the mobility at $E_{F}$ to the point where thermal excitation of carriers across the mobility gap becomes the dominant transport mechanism (Fig. 6.2c).

A plot of the $\log \sigma$ as a function of $1 / T$ was first investigated to determine the dominant transport mechanism in shock compressed liquid nitrogen and appears in Fig. 6.3.

It should be emphasized that, unlike the metallic vapors investigated by Hensel and others along isotherms and isobars, these results are along a Hugoniot, where there are simultaneously large 


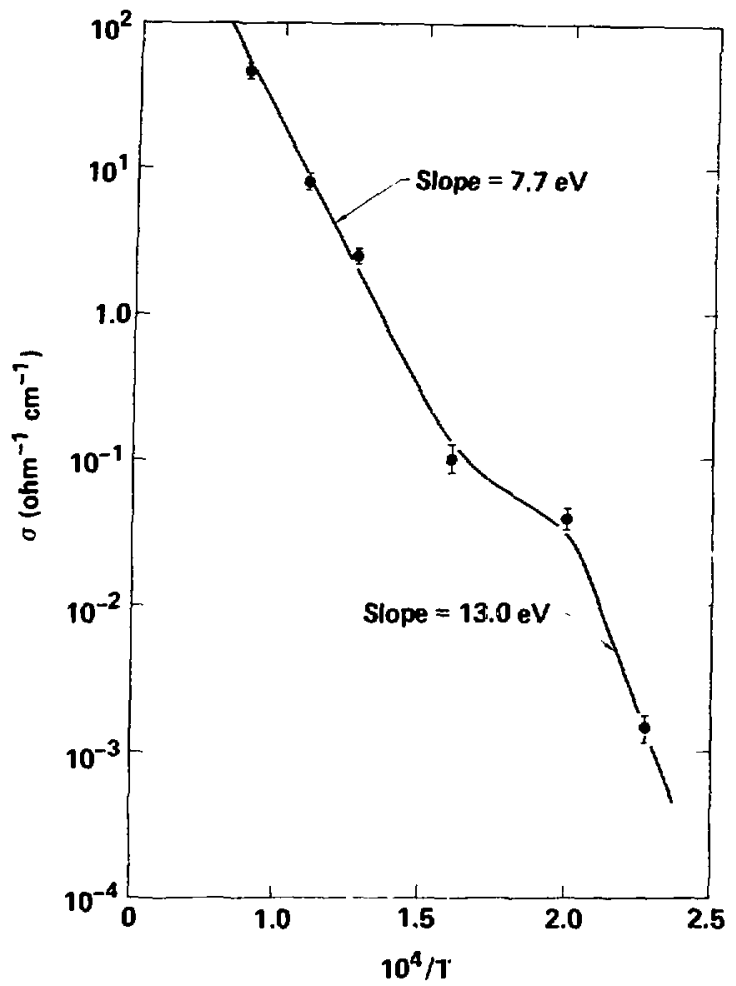

Fig. 6.3 Arrhenius plot of electrical conductivity for shock compressed liquid nitrogen.

changes in both density and temperature. The slope for low temperatures is around 73 ev (comparable to the ionization energy of a free molecule). followed by a kink near $10^{4} / T \sim 1.8(\sim 30 \mathrm{GPa})$, and finally, a slope for the higher temperatures around $8 \mathrm{ev}$. The notion of activation along a Hugoniot in the hopping regime is misleading and can be cemonstrated with the aid of a o-T-p diagram for a hypothetical metallic vapor (Fig. 6.4). 


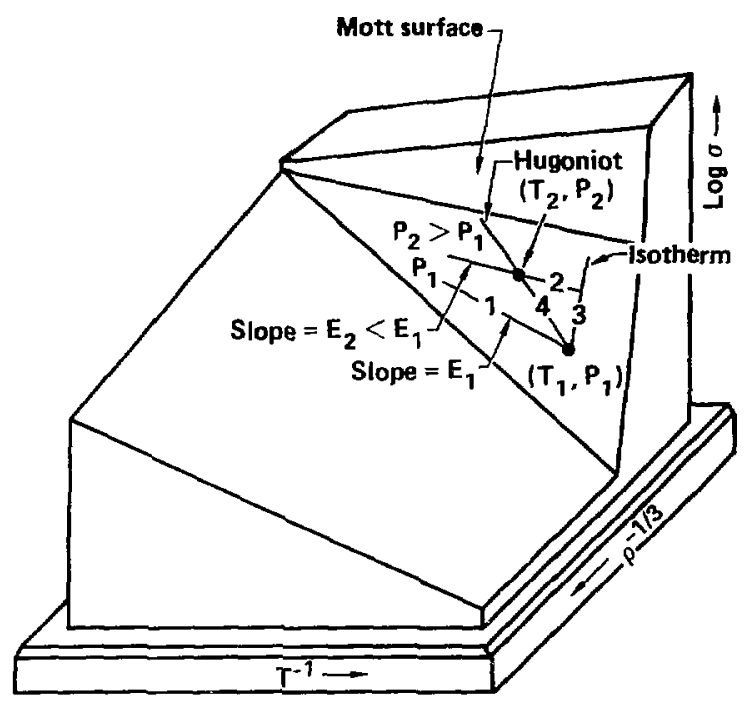

Fig. 6.4 o-T-P diagram for a hypothetical metallic vapor.

If the temperature is increased starting at $\left(T_{7}, P_{7}\right)$, while the pressure is held constant, then the conductivity will increase exponentially with a slope which corresponds to an activation energy, say, $E_{1}$. If the same experiment is repeated at a higher pressure, $P_{2}$, the conductivity increases with a characteristic slope, say, $E_{2}$ (which is generally less than $E_{1}$ ). In either case an activation energy can be inferred from an Arrhenius plot; at least locally. Also notice that increasing the pressure along curve 3 , thus decreasing the interatomic separation, will effectively increase the conductivity with a slope representing a measure of the wave function extent. In a shock compression process, the material could conceivably proceed along a path like curve 4 , whose slope is the sum of contributions due to compression and heating. Hoshino, for example, heated selenium along a 500 and 1200 bar isobar. Each of these 
gave an activation energy of about $2 \mathrm{ev}$. If an activation energy were inferred, based on two pressure and temperature pairs, $\left(P=500,10^{4} / 1=8\right)$ and $\left(P=1200,10^{4} / T=7\right)$, an activation energy nearly $30 \%$ higher would be obtained. For this reason slopes of in a plotted as a function of $1 / T$ along Hugoniots in the hopping regime can give erroneous activation energies.

In a hopping regime a plot of $\log \alpha$ as a function of a should

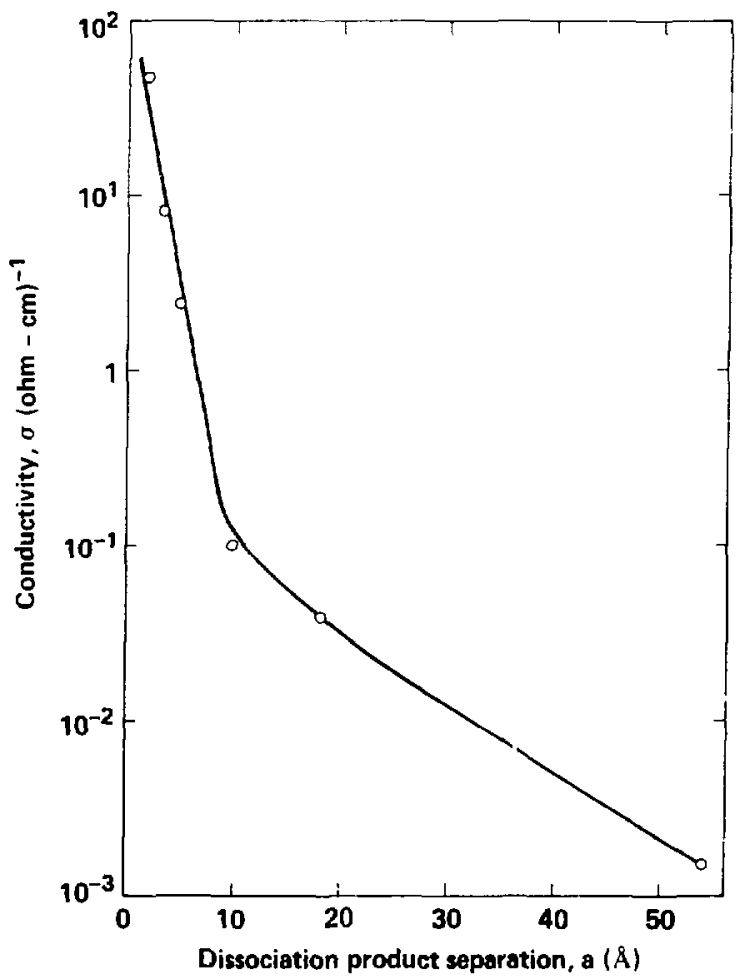

Fig. 6.5 Dependence of electrical conductivity of shock compressed liquid nitrogen on the dissociation product separation. 
prove useful. This is shown in Fig. 6.5, where the intermolecular separation between monatomic nitrogen species is defined as

$$
a=\left(\frac{3}{4 \pi N_{s}}\right)^{1 / 3} .
$$

The concentration of monatomic species, $\mathrm{H}_{S}$, is related to the fractional dissociation, $x_{2}$, degree of compressibility, $x$, initial density, $P_{0}$, molecular weight, $M$, and Avogadro's number, $N_{A}$, through

$$
\left.\begin{array}{c}
N_{5}=2 x_{2} N \\
N=\frac{x_{0} N_{A}}{H}
\end{array}\right\}
$$

The plot shows the same qualitative features and trends observed for metal vapor, that is a low-density region with a weak dependence in interparticle separation (a $>10 \AA)$, and a higher density region displaying a rapid increase in electrical conductivity with decreasing separation.

Seager and Pike studied the hopping conduction problem by computing the conductivity of a random lattice with interlattice site conductances depending exponentially on their separation. ${ }^{84}$ Identifying the critical radius for overlapping spheres $r_{s}=0.7 \mathrm{a}$, they were able to determine the critical conductance at which form the first conducting chains of infinite extent. The conductance was given by

$$
\sigma \sim \mathrm{e}^{-(1.4) \frac{2}{\lambda} \mathrm{a}}
$$

where $\lambda$ was identified as the radius of the localized wave function. Identifying the slope in this work $(-1 / 1.3)$ with $-2(1.4) / \lambda$ gives an 
estimate for the wave function radius, $\lambda \sim 3.6 \AA$.

Equation (6.7) can be applied to determine the minimum metallic conductivity expected for this material at $60 \mathrm{GPa}$ for $\mathrm{g} \sim 0.2$ and a $1.9 \AA$. The result is $\sigma=680 \Omega^{-1} \mathrm{~cm}^{-1}$ which is about 14 times larger than the measured value.

The measured data for the electrical conductivity of liquid nitrogen are consistent with a model which describes the low dynamic pressure $(P \leq 30 \mathrm{Ga})$ region is predominantly involving thermal activation of carriers across a psuedo gap, and a high temperature region where thermally-activated hopping takes over. This latter regime generally precedes the onset of metallic-like electronic transport.

\subsection{Oxygen Electrical Conductivity}

It was stated in Chapter 2 that good agreement was found between Hugoniot EOS measurements and Ross' calculations which incorporated a corresponding states scaling of an argon potential. This differs from the circumstances found for nitrogen where dissociation played an important role in the electrical conduction process.

An Arrhenjus plot is shown in Fig. 6.6 which combines Ross' calculated temperatures with the electrical conductivity data for oxygen. The remarkable feature of this plot is the nearly constant activation energy over all but the highest temperatures. The slope corresponds to an energy of $4.6 \mathrm{ev}$. Molecular oxygen is known to have an allowed 


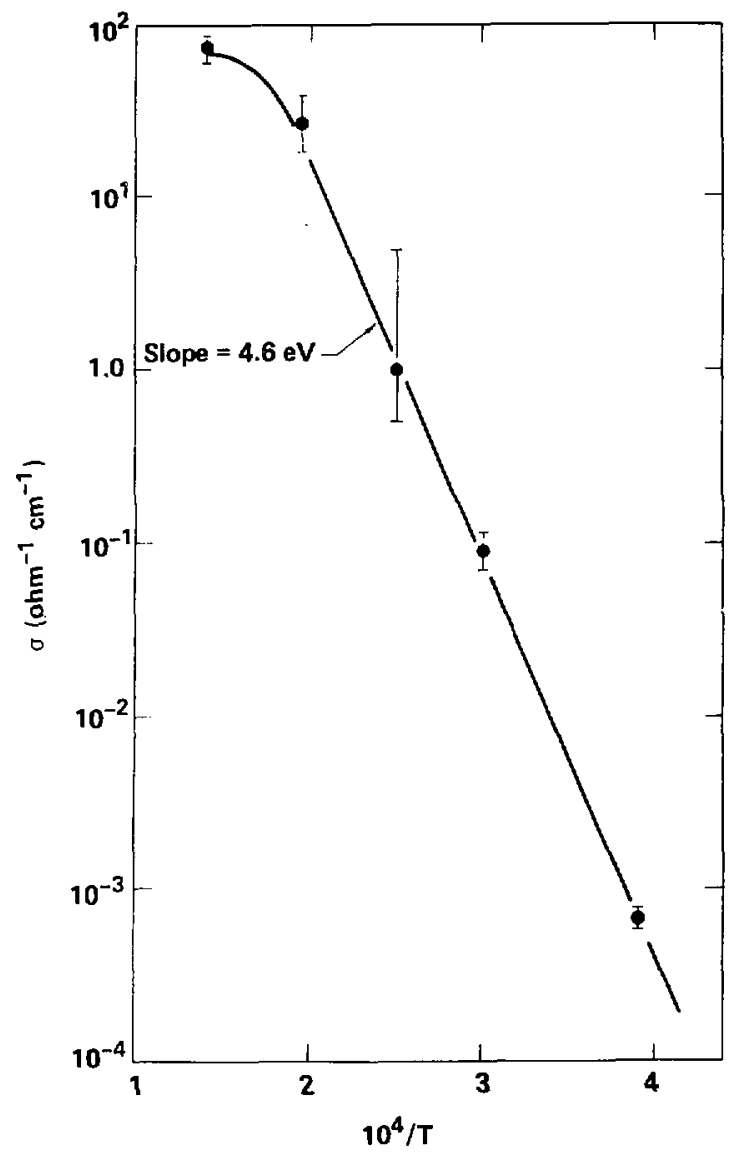

Fig. 6.6 Arrhenius plot of electrical conductivity for shock compressed liquid oxygen.

transition $x^{3} \Sigma_{g}^{-} \rightarrow B^{3} \Sigma_{u}^{-}$with a corresponding energy of $6.1 \mathrm{ev}{ }^{61}$ The spectroscopic symbols, $X$ and $a$, indicate the ground state and second electronic energy levels, while ${ }^{3} \Sigma_{\mathrm{u}}^{-}$and ${ }^{3} \Sigma_{\mathrm{g}}^{-}$indicate the spin and symmetry properties of the state. 


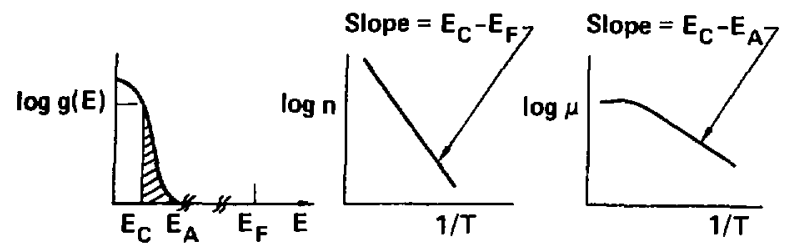

Fig. 6.7 Schematic representation of temperature dependence for carrier concentration and mobility for carriers in a band with a density of states shown on left.

These results can be interpreted in terms of change transport in extended states beyond a mobility edge. See Fig. 6.7 which is reproduced from Mott. ${ }^{81}$ Electrical conductivity for disordered materials in the high temperature regime such as shock-compressed liquid oxygen involve transport of carriers at a mobility edge $E_{c}$. The activation energy is $E_{c}-E_{F}$. The drift mobility is trap-limited at these temperatures and shows a temperature dependence with an activation energy $E_{C}-E_{A} \equiv \Delta E$. The distribution of tail states, which was discussed in Section 6.2, is due to fluctuations in the intermolecular potential and can be appreciable for hot liquids. For materials containing one or more elements from Group $V I$ of the Periodic Table the width of tail states at room temperature lies in the neighborhood 0.1-0.5 ev and increases, approximately, linearly with emperature. ${ }^{81,82}$ Another characteristic of extended state conduction is that mobility is weakly dependent on pressure (or density).

Suppose the density of states and the positions of $E_{C}, E_{A}$ and $E_{F}$ do not change appreciably with temperature and pressure. In this case, the Arrhenius plot shown in Fig. 6.6 represents a true activation energy equivalent to a measured electrical conductivity dependence along an 
isobar. If the current-carrying species are electrons and holes, thermally excited from the valence to the conduction band and the fermi energy is at mid-gap, then $E_{C}-E_{F} \sim 3 \mathrm{ev}$. So, the difference between the measured activation energy $(4.6 \mathrm{ev})$ and $E_{c}-E_{F}$ may represent the tail width $\Delta E=1.6 \mathrm{ev}$.

For a tail-state distribution $g(E) \sim\left(E-E_{A}\right)^{n}$ the drift mobility becomes

$$
\mu \sim \mu_{0}\left(\frac{\Delta E}{k T}\right)^{n} e^{-\frac{\Delta E}{k T}},
$$

where $\mu_{0}$ is the drift mobility at the mobility edge, $E_{c} \cdot{ }^{81}$ for higher temperatures the drift mobility saturates, and shows a weaker $(1 / T)^{n}$ dependence. The magnitude and shape of the saturation at higher temperatures are consistent with this interpretation.

The pre-exponential factor in the conductivity, $\sigma=\sigma_{0} \mathrm{e}^{-E / K T}$ where $E=E_{C}-E_{F}+\Delta E=4.6 \mathrm{ev}$, is $\sigma_{0}=7 \times 10^{5} \Omega^{-1} \mathrm{~cm}^{-7}$ and is a magnitude characteristic of transport in the extended state regime.

An upper bound for the charge carrier concentration can be estimated by assigning a minimum value for the saturated extended state mobility (frequent diffusion-type scattering). Applying Cohen's expressions (Eqs. (6.20) and (6.21), presented in the next section) for $a=1.7 \AA$ and $T=$ $5000 \mathrm{~K}$ gives $\mu \sim 1 \mathrm{~cm}^{2} / v \cdot \mathrm{sec}$. Since $\sigma=n e_{\mu}$, it follows $n \sim 2 \times 10^{20}$ $\mathrm{cm}^{-3}$ which is about 1 electron per 300 oxygen molecules. 


\subsection{Benzene Electrical Conductivity}

Here, a madel for the observed electrical conductivity of benzene is described. Three assumptions are made: (1) Ree's hydrocarbon model, which assumes that benzene decomposes to form a two phase mixture of diamond and molecular hydrogen, is valid. (2) The electronic properties of the diamond phase are similar to those of the amorphous forms of other Group IV elements. (3) The diamond structure which is formed, besides being amorphous, will also contain voids which can exhibit a connectivity-dependence in the electronic properties characteristic of percolation problems.

Hugonfot measurements performed on benzene support Ree's model which predicts that benzene decomposes ( $P \geq 14 \mathrm{GPa}$ ) into a mixture which is predominately carbon in a diamond-like solid phase and molecular hydrogen in a fluid phase. It is expected that agreement between the simple two-phase model and the measured final state should improve with higher dynamic pressures. Any other $H: C$ species present, particularly at lower shock pressures, are expected to play a minor role in determining the electrical properties. Also, molecular hydrogen will be treated as an inert (insulator) material since the degree of electronic excitation and/or ionization for the conditions reported here should be negligible.

Comparisons with diamond can be made with germantum and silicon, because these materials all belong to the the same column (IV) of the Periodic Table. An extensive amount of information has been gathered on these materials over the last 20 years. Group IV-type materials, such as 
carbon in the diamond phase, are tetrahedally coordinated, covalent materials. The outer orbitals ( $2 p$ and $2 s$ ) determine the bonding properties. As the interatomic separation decreases the energy levels of the individual atoms interact and broaden into bands. Eventually, upon further decrease in separation, the states mix and hybridize to form four equivalent covalent bonds which are directed toward the corners of a tetrahedron. The sp orbitals interact in such a manner as to form bonding and antibonding states. The lower band (valence) is completely filled, since there are four valence electrons per atom, and the upper band (conduction) is empty.

The nature of these bonds which detemines short-range order and influences the long-range structure. With sufficiently slow condensation, the atoms form a lowest-energy configuration where each atom is bonded to four other atoms thus forming a giant $C_{n}$ molecule. The nearest-neighbor separation is $1.54 \AA$. The angle formed by an atom and its two nearest neighbors is about $109.8^{\circ}$. Since each carbon atom must itself be surrounded by such a tetrahedron, there are 12 second-nearest neighbors 2.52 \& away.

It was stated in Chapter 2 that the cooling path followed in a condensation process (that is, whether the crystalline or glassy solid phase is formed), is determined by the cooling rate. Constraints, imposed by the degree of coordination between nearest neighbors, favor the lowest -energy crystalline configuration. ${ }^{35,78,82}$ Because Group IV materials have a large coordination number, extremely fast deposition rates are required to form an amorphous structure. 
The first two peaks in the radial distribution function for vapor-deposited silicon shows a structure similar to the crystalline form indicating that the nearest-neighbor environment of a silicon atom in an amorphous structure is also approximately tetrahedral with about the same bonding distance as in the crystalline form. The second peak has a greater width indicating a disorder-induced spread in the second-neighbor distance, but with a mean value similar to the crystalline case. There is no clear indication of a third peak, reflecting the disorder in the geometry and topology of amorphous structures. Amorphous structures have more freedom to vary the angle between adjacent bonds, $\theta$, than the bond lengths, $a$, and coordination number, $z$. Continuous random networks (CRH) can be constructed satisfying local requirements, with small spreads in $\theta$, and still lack long-range order. ${ }^{8}$ CRHs are idealizations in that they are perfectly connected. Amorphous materials produced by vapor deposition, however, are not homogeneous but contain voids between $5-40$ $A$ in diameter, depending on the details of deposition. Evaporated germanium, for example, contains $10^{18}$ voids $/ \mathrm{cm}^{3}$ with an $17 x$ density deficit. Where there are voids there usually are broken bonds which can be detected with electron spin resonance. A sketch of the density of states for a typical amorphous Group IV material with voids is show in Fig. 6.1b.

This model for the density of states together with Eq. (6.5) can be used to calculate the electrical conductivity of a fully connected diamond lattice of finite temperatures. Let oxt denote conduction due to delocalized electrons. If conduction takes place by e?ectrons sufficiently far from $E_{F}$ and a shaip Mott-like mobility edge is 
assumed, then it follows that

$$
\sigma_{\text {ext }}=e g\left(E_{c}\right) \mu_{e} K T \exp \left[-\left(E_{c}-E_{F}\right) / K T\right]
$$

which is of the form

$$
\sigma=n \text { e } \mu .
$$

The charge carrier concentration is given by

$$
n=g\left(E_{c}\right) K T \exp \left[-\left(E_{c}-E_{F}\right) / K T\right] \text {. }
$$

In diamond the conduction band width is about $5 \mathrm{eV}$. The corresponding density of states at $E_{c}, g\left(E_{c}\right)$, can be estimated by recognizing that there are fou: valence electrons per atom and about $2 \times 10^{23}$ atoms per unit volume. Therefore, $g\left(E_{c}\right) \sim 2 \times 10^{23} \mathrm{~cm}^{-3} \cdot \mathrm{ev}^{-7}$. If Mott's expression for the minimum mobility for extended state conduction is used,

$$
\mu_{e}-\frac{e a_{E}^{2} B \Gamma}{3 \hbar K T},
$$

where $a_{E}$ is the distance over which the wave function loses phase menory (corresponding to a lattice parameter, $3 \lambda$ ), $B$ is the band width and $\Gamma$ is a constant which depends on the coordination number (typically $1 / 3$ for coordinator 4) then $\mu_{e} \sim 10 \mathrm{~cm}^{2} / \mathrm{volt} \cdot \mathrm{sec}$ at room temperature. This mode of transport is described by Cohen as diffusive (Brownian motion) transport and gives ar expression 48

$$
\mu_{e}^{B M} \sim \frac{e D}{K T} .
$$

where the diffusion coefficient, $D$, is related to the electron frequency, el, and interatomic spacing, a, through

$$
D \sim \frac{1}{6} \mathrm{ues}^{\mathrm{a}^{2}} .
$$


The values of $\mu_{e}$ given by Mott's and Cohen's expressions are comparable. This mode of extended state transport can also be described as one where the electron mean-free-path is on the order of/or smaller than an interatomic separation. The observed activation energy at high temperatures for extended state conduction is not $E_{c}^{\prime}-E_{v}^{\prime}$ because the mobility gap is a function of temperature. Phonons contribute a term to electron self-energies. A linear dependence is typical.

$$
E_{C}^{\prime}-E_{v}^{\prime} \sim \Delta E_{G}^{\prime}-Y^{T}
$$

where the temperature coefficient, $r$, is observed to lie between

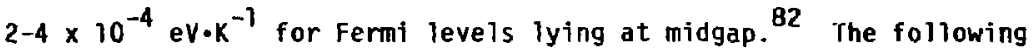
expressions for the carrier concentration and mobility,

$$
\begin{aligned}
& n=2 \times 10^{23}(K T) \exp (\gamma / K) \exp \left[-\left(E_{C}-E_{F}\right) / K T\right], \\
& \mu=\mu_{0}(300 K)\left(\frac{T O}{T}\right),
\end{aligned}
$$

where $\mu_{0}(300 \mathrm{~K})=10 \mathrm{~cm}^{2} / \mathrm{v} \cdot \mathrm{sec}, T_{0}=300 \mathrm{~K}, E_{F}=\frac{E_{V}+E_{C}}{2}$ and $\gamma=2 \times 10^{-4}$, can be substituted into $\mathrm{Eq} .(6.10)$ to give the minimum extended-state conductivity for a fully connected amorphous diamond. A plot of oxt is shown in Fig. 6.8.

It has been stated that similarities could be expected in the structure and hence the density of states between vapar deposited silicon and the diamond form of carbon produced in a shock process. However, in addition to being amorphnus, the material also contains voids. Transport properties are sensitive to the connectivity of the lattice structure. The density deficit or void fraction is a measure of the lack of 


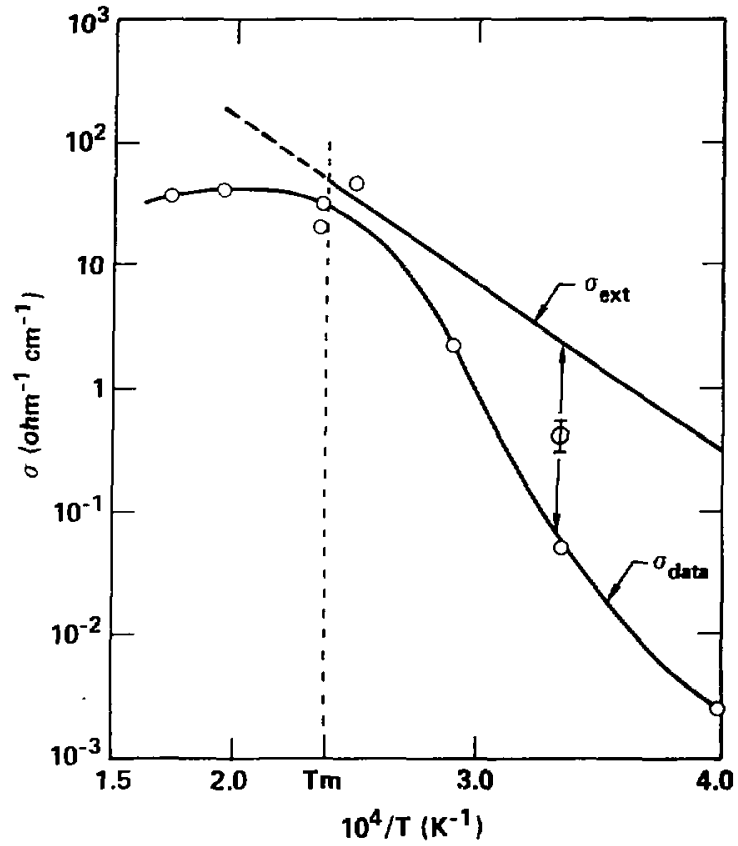

Fig. 6.8 Arrhenius plot of electrical conductivity for shock compressed benzene showing data and calculated results, assuming a fully connected, semiconducting diamond.

connectivity and for this reason will now be calculated. The volume fraction of diamond (or void fraction) to total volume can be estimated by recognizing the final composition is 50\% carbon. The number of carbon atoms per unit volume will be

$$
N_{C}=\frac{6 x_{B} P_{B} N_{A}}{M}
$$

where $x_{B}$ is the degree of compression of berizene $\left(\rho / \rho_{B}\right), M$ is the molecular weight of benzene (78), $N_{A}$ is Avogadro's number, and $\rho_{B}$ is the 
initial density $(.88 \mathrm{~g} / \mathrm{cc})$. The factor 6 is included since there are 6 carbon atoms per benzene molecule. The unit cell for a diamond lattice measures 3.567 and contains 8 atoms. Therefore, the concentration of carbon atoms becomes

$$
N_{0}=1.76 \times 10^{23} x_{0}\left(\mathrm{~cm}^{-3}\right),
$$

where $x_{D}$ is the degree of compression for diamond. Since the EOS for diamond is known, $x_{D}$ can be determined for any chosen dynamic pressure. The fraction of diamond-like carbon in the total volume is therefore

$$
x=\frac{N_{C}}{N_{0}} .
$$

Another method to determine $x$ requires the EOS of hydrogen. The bulk density, $S(x)$, can be expressed in terms of the density of diamond, $x_{D} P_{D}$, and the density of hydrogen, $x_{H} P_{H}$, to give

$$
S(x)=x_{P_{D}} x_{D}+(1-x) p_{H} x_{H}
$$

and can be inverted to give $x$. Table 6.2 gives the compressibility of benzene and diamond, the volume fraction of diamond and the percentage decrease in lattice parameter (assuming isotropic compression) as a function of pressure. The factor of two discrepancy in the density suggests that the material contains defects and/or macroscopic voids.

It is expected that the bulk conductivity, $\sigma_{\text {BuLK, will be less }}$ than the conductivity for a fully connected network. There are three basic structural regimes to consider for composite materials: 
Table 6.2. Degree of compression for benzene and diamond used to calculate volume fraction and lattice parameter change of diamond as a function of shock pressure.

\begin{tabular}{ccccc}
\hline$P(6 \mathrm{~Pa})$ & $X \mathrm{~B}$ & $\mathrm{XD}$ & $x$ & $-\Delta \mathrm{A}(\boldsymbol{X})$ \\
\hline 21.1 & 2.248 & 1.022 & 0.507 & 0.7 \\
24.9 & 2.318 & 1.028 & 0.519 & 0.9 \\
30.0 & 2.397 & 1.036 & 0.533 & 1.2 \\
37.2 & 2.487 & 1.049 & 0.546 & 1.6 \\
41.9 & 2.538 & 1.057 & 0.553 & 1.9 \\
\hline
\end{tabular}

1. The conductor regime; where the volume fraction of the conductor, $x$, is large and forms a continuum with intermixed insulator inclusions.

2. The insulator regime; $x$ is small and represents an inversion of the conductor regime. Electrical transport occurs primarily through the insulator by tunneling or thermionic emission between conducting islands.

3. The transition region; this is where a structural inversion takes place and displays large changes in the electrical conductivity for small changes in $x$. The number of contacts per particle is becoming sufficiently large, with positive changes in $x$, to produce networks of very long chains. This is a percolation phenomenon. A critical percolation threshold exists that depends on a critical percolation probability (lattice-dependent) and the potential number of contacts possible per atom (coordination-dependent). The author believes that the discrepancy between $\sigma_{\text {DATA }}$ and $\sigma_{E X T}$ is due to an increase in the 
number of conduction paths through the diamond matrix as $x$ increases. This behavior has been observed by Abeles and coworkers, ${ }^{85}$ Malliaris and Turner, 86 and Gurland 87 for various matrix combinations. They onserved a critical transition in the percolation process at some critical void fraction $(1-x \sim 0.4-0.6)$, which depended on the particular material. The volume fraction of diamond, in this work, corresponds to these critical composition values $(x \sim 0.5)$. Also the rapidly approaching agreement between the measured conductivity and calculated values, assuming a perfectly connected network $(x=1)$, is consistent with this interpretation. The author defines a dimensionless geometrical factor, $\Phi$, given by

$$
\sigma_{\text {DATA }}=\phi^{-1} \sigma_{E X T}
$$

which is anaiagous to the geometrical factor introduced by Abeles and coworkers and represents a measure of the connectivity between conducting particles (Fig. 6.9). The dependence displays features similar to the work of Abeles and coworkers on $\mathrm{Ni}-\mathrm{SiO}_{2}$ and $\mathrm{Au}-\mathrm{Al}_{2} \mathrm{O}_{3}$ systems.

The final point the author wishes to discuss is the prospect of melting. The vanishing temperature coefficient of resitivity near $4000 \mathrm{~K}$ (Fig. 6.8) is characteristic of a metallic transition. This is also the region Van Vechten proposes as the melting temperature of diamond ( $T$ $4100 \mathrm{~K}$ and $\mathrm{P}-46 \mathrm{GPa})$. A phase diagram for a number of hydrocarbons, including the predicted diamond melting line, can be found in Ree's paper. $^{56}$ One is generally accustomed to observing an abrupt increase In the electrical conductivity upon melting Group IV-type materials. In Germantum, for example, the conductivity increases by nearly a factor of 


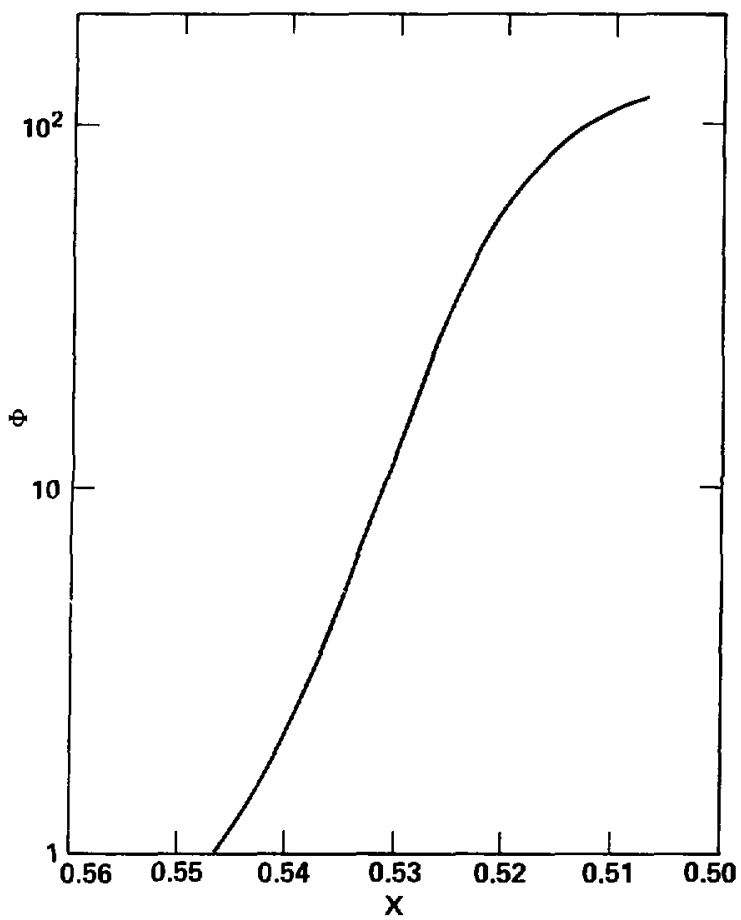

Fig. 6.9 Geometry factor of the resistivity as a function of the volume fraction of diamond.

20 from $1000 \Omega^{-1} \mathrm{~cm}^{-1}$ to $16000 \Omega^{-1} \mathrm{~cm}^{-1}$. At this transition the number of carriers increases about 4 orders-of-magnitude, (corresponding to 3 carriers per atom) and the drift mobility decreases almost 3 orders-ofmagnitude, to around $1 \mathrm{~cm}^{2} /$ volt.sec. The number of carriers, using Eq. $(6.23)$, is $4 \times 10^{20} \mathrm{~cm}^{-3}$. If there are 4 carriers per carbon atom in the metallic state, then the concentration of carriers becomes $10^{23}$. A factor of $10^{3}$ increase, could be of fset by a factor of $10^{3}$ decrease in the mobility. This seems doubtful since it has been assumed that the regime 
is one of minimum extended state mobility. Another way to offset the $10^{3}$ increase in carriers would involve a change in connertivity, $\phi$. The density of germanium increases by $4 \%$ when it melts. This should effect ${ }_{D}$ and hence, $x$. If it is assumed that diamond aiso increases in density by $4 \times$ then $\times$ will change from its diamond lattice value of 0.55 to 0.53 and - (from Fig. 6.9) decreases by a factor of 20 . A $4 \%$ change in specific volume should be within the limits of resolution for Hugoniot measurements previously reported for benzene. There does not seem to be any indication of a phase change in this region. Other than a gradual transition to a temperature-independent conductivity, which is characteristic of "dirty" metals with strong scattering, the data presented do not strongly support Van Vechten's proposed melting temperature for diamond. 
The fractional error for the electrical conductivity is given by

$$
\frac{\Delta \sigma}{\sigma}=\frac{\Delta \rho}{\rho},
$$

where,

$$
\sigma=\frac{1}{\rho}
$$

and

$$
\rho=\frac{R}{F} \quad .
$$

Assuming independent random error in $F$ and $R$, it follows that

$$
(\Delta \rho)^{2}=\rho^{2}\left[\left(\frac{\Delta R}{R}\right)^{2}+\left(\frac{\Delta F}{F}\right)^{2}\right] .
$$

In a few rare cases, particularly when stainless steel electrodes were used to measure high conductivities, the measured resistance $R^{\prime}$ would be the sum of electrode resistivities $R_{E}$ and sample resistivity $R, R_{E}$ and $\Delta R_{E}$ for the stainless and copper electrodes were $0.034 \pm 0.0004$ ohms and $0.002 \pm 0.0004$ ohms, respectively. This would introduce ar, uncertainly in $R$ as

$$
(\Delta R)^{2}=\left(\Delta R^{\prime}\right)^{2}+\left(\Delta R_{E}\right)^{2}
$$

The analysis depends on which circuit is considered. For a constant-current circuit, the analysis is based on the error associated with Eq. (4.5) and the measured parameters $V_{x}, V_{0}$ and $R_{S H}$. The fractional uncertainty in the total resistance, $R^{\prime}$, becomes 


$$
\left(\Delta R^{\prime}\right)^{2}=\left(R^{\prime}\right)^{2}\left[\left(\frac{\Delta R_{s}}{R_{s}}\right)^{2}+\left(\frac{V_{0}}{V_{x}}\right)^{2}\left(\frac{\Delta V_{x}}{V_{x}}\right) \frac{1}{\left(\frac{V_{0}}{V_{x}}-1\right)^{2}}\right] .
$$

The uncertainty in the shunt resistance is assigned a value of $7 \%$ based on a measured reproducibility of resistance values of the precision resistors after cryogenic cooling. The uncertainty in the initial deflection, $v_{0}$, can be ignored. The uncertainty in the final deflection is largely subjective and judgment is based on signal-to-noise ratios and clarity in definition of the signal break when the shock wave reached the electrode base. In all cases conservative values are assigned. Eq. (A.6) also implicity demonstrates the dependence of $\Delta R$ on the ratio $v_{0} / v_{x}$ and is minimized for $v_{0}=2 v_{x}$.

A linear-regression analysis is used for the constant-voltage circuit scope calibration. ${ }^{89}$ In this case the standard deviations for the fitting parameters of Eq. (4.4), $S_{a}$ and $S_{b}$, and the calculated value from the fit, $x_{0}$, and its standard deviation, $S_{0}$, are needed to determine the fractional uncertainty in the measured resistance. The standard deviation for the calculated value, $x_{0}$, is

$$
s_{0}^{2}=\frac{s_{y}^{2}\left[\begin{array}{c}
k \\
\Sigma \\
i
\end{array} x_{1}^{2}-2 x_{0} \sum_{i}^{k} x_{i}+k x_{0}^{2}\right]}{(k-1) 0}
$$

where $k$ is the number of standard resistors, $S_{y}$ is the standard deviation of the difference of scope deflections between measured and fit values,

$$
S_{y}^{2}=\frac{\Sigma(\delta y)^{2}}{K-1}
$$


and $D$ is the Jacobian

$$
k \underset{i=1}{k} x_{i}^{2}-\left(\sum_{i=1}^{k} x_{i}\right)^{2}
$$

The error associated with the linear fit in determining the resistance is expressed

$$
\left(\Delta x_{0}\right)^{2}=\left(\frac{y_{0}}{b}\right)^{2}\left[\left(\frac{s_{0}}{y_{0}}\right)^{2}+\left(\frac{S_{b}}{b}\right)^{2}\right]+\left(\frac{a}{b}\right)^{2}\left[\left(\frac{s_{a}}{a}\right)^{2}+\left(\frac{s_{b}}{b}\right)^{2}\right] .
$$

where $y_{0}$ is the measured deflection value,

$$
\begin{aligned}
x_{0} & =\frac{y_{0}-a}{b}, \\
s_{a}^{2} & =\frac{s_{y}^{2} k}{(k-1)} \sum_{i=1}^{k} x_{i}{ }^{2} . \\
\text { and } s_{b}{ }^{2} & =\frac{s_{y}^{2} k^{2}}{(k-1) B} .
\end{aligned}
$$

The uncertainty in the calculated resistance (Eq. (4.4)) becomes

$$
\left(\Delta R_{0}\right)^{2}=\left(R_{0}\right)^{2}\left[\left(\frac{\Delta R_{2}}{R_{z}}\right)^{2}+\left(\frac{V_{0} R_{z}}{R_{0}}\right)^{2}\left(\frac{\Delta X_{0}}{X_{0}}\right)^{2}\right] \text {, }
$$

where

$$
R_{0}=R_{z}\left[\frac{v_{0}}{x_{0}}-1\right] .
$$

Finally, the fractional uncertainty in the conductivity is

$$
\left(\frac{\Delta \sigma}{\sigma}\right)^{2}=\left(\frac{\Delta \rho}{\rho}\right)^{2}=\left[\left(\frac{\Delta R_{0}}{R_{0}}\right)^{2}+\left(\frac{\Delta F}{F}\right)^{2}\right] \text {. }
$$




\section{APPENDIX B. REFERENCE ENERGY FOR 1-BUTENE}

The Initial energy, $E_{0}$, used in CHEQ for calculating the Hugoniot and which appears in the third Hugoniot relation, is referenced to the reduced form of molecular hydrogen and graphite at $298.15 \mathrm{~K}$ and 1 atmosphere pressure. The heat of formation of liquid 1 -butene at $262.6 \mathrm{~K}$ and 1 atmosphere pressure was not available so the following procedure was necessary.

The reduction of the initial liquid 1-butene $\left(\mathrm{CH}_{2}=\mathrm{CH}-\mathrm{CH}_{2}-\mathrm{CH}_{3}\right)$ sample at $262.6 \mathrm{~K}$ can follow paths $1 \rightarrow 2 \rightarrow 3$ as shown in Fig. B.1. The energy difference between the end states $\Delta E$ is

$$
\begin{aligned}
\Delta E= & E\left(4 \text { moles of } H_{2}+\text { graphite a } 298.15 \mathrm{~K}\right)-E(1 \text { mole of 1-butene } \\
& \quad(262.6 \mathrm{~K}) \\
= & \Delta E_{1}+\Delta E_{2}+\Delta E_{3} .
\end{aligned}
$$

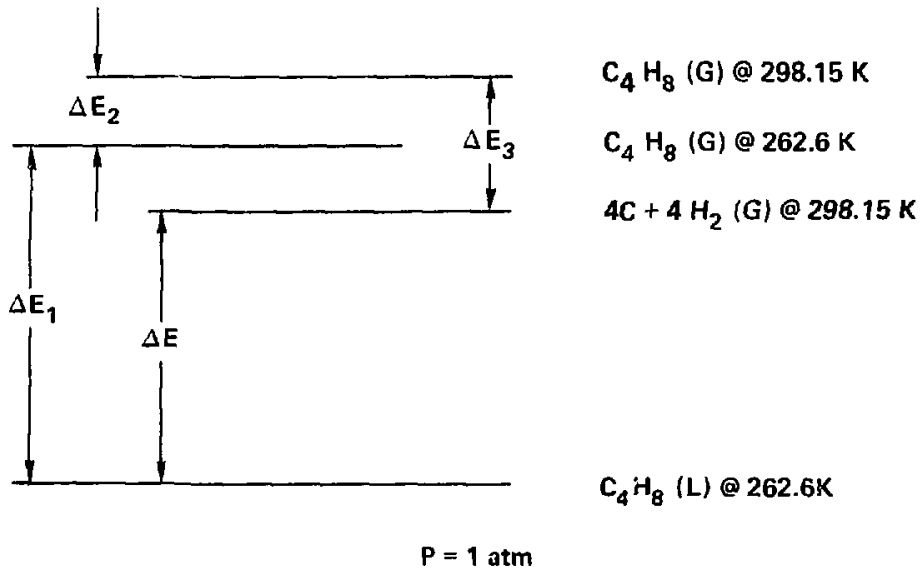

Fig. B.1 Determining the reference state for 1 butene. 
Calculating the energy difference along each path can be simplified by recognizing some useful relations between the energy and enthalpy $H$,

$$
E=H-P V .
$$

For example, the energy difference between state 1 and 2 (path ?) car be expressed

$$
(\Delta E)_{1}=(\Delta H)_{1}-\Delta(P V)=L_{12}-\left[(P V)_{G}-(P V)_{L}\right] \simeq L_{12}-R T \text {, }
$$

where $L_{12}$ is the latent heat of vaporization at $262.6 \mathrm{~K}$. Also, use was made of the fact that $(P V)_{G} \gg(P V)_{L}$. Substituting $L_{12}=5331$ $\mathrm{cal} / \mathrm{mole} \mathrm{e}^{90}$ into $\mathrm{Eq}$. (B.3) gives $(\Delta \mathrm{E})_{1}=4809 \mathrm{cal} / \mathrm{mole}$.

The energy difference along path 2 is

$$
(\Delta E)_{2}=\delta H_{2}-\Delta(n R T) \text {, }
$$

where

$$
\delta H_{2}=\int_{262.6}^{298.15} C_{p} d T
$$

and

$$
\Delta(n R T)=n R \Delta T .
$$

Data for the specific heat at constant pressure $C_{p}$ for 1-butene are found in Ref. 75 and can be fitted to a polynomial. The results are $\delta H_{2}=427 \mathrm{cal} / \mathrm{mole}$, and combined with $\mathrm{nR \Delta T}=67 \mathrm{cal} / \mathrm{mole}$ give $(\Delta E)_{2}=360 \mathrm{cal} / \mathrm{mole}$.

The energy difference along path 3 is

$$
\Delta H_{f}^{0}, 298.15-\Delta(n R T) \text {, }
$$

where $\Delta H_{f}^{0}, 298.15(=280 \mathrm{cal} / \mathrm{mole})$ is the heat of formation for 1-butene at $298.75 \mathrm{~K}$ and $\Delta(\mathrm{nRT})=3 \mathrm{RT}$, since there is a net change of 3 moles in 
going from 1 mole of 1-butene to 4 moles of molecular hydragen plus graphite. ${ }^{90}$ it follows that $(\Delta E)_{3}=-1497 \mathrm{cal} / \mathrm{mole}$.

Combining these results give the total energy difference

$$
\Delta E=-3672 \mathrm{cal} / \mathrm{mole}=2.738 \times 10^{-3} \frac{\mathrm{mbar} \cdot \mathrm{cm}^{3}}{\mathrm{~g}} .
$$

The negative sign is included because the zero reference is defined as the state $\mathrm{H}_{2}+\mathrm{C}$. 
What shouid the signal look like for a constant-current circuit (Fig. 4.8b) and how does it compare to what is observed? Consider experiment $\mathrm{NC}-8$ where,

$$
\begin{aligned}
& R_{s}=1 \mathrm{ohm}, \quad F=4.2 \mathrm{~cm}^{-1}, U_{s}=0.82 \mathrm{~cm} / \mathrm{\mu s} \text {, and } \\
& \Delta t_{\text {elec }}=t_{f}-t_{0}=0.11 \mathrm{\mu s} .
\end{aligned}
$$

Figure $[$.$] shows a reproduction of the signal record. The geometrical$ fringing field correction $F$ is defined as

$$
F=\frac{\ell}{A} f .
$$

Since $\ell=0.7 \mathrm{~cm}$ and $A=0.07 \mathrm{~cm}^{2}, f$ is calculated to be 0.39 .

Suppose $f=$ constant from the moment the shock wave reaches the electrode $t i p\left(t=t_{0}\right)$ to shock arrival at the electrode base $(t=$ $\left.t_{f}\right)$. The time-dependence of the exposed electrode area is

$$
\begin{aligned}
A=A(t) & =(0.1)(0.82) \mathrm{cm}^{2} . \\
{[t] } & =\mu \mathrm{S},
\end{aligned}
$$

so the resistance of the sample $R_{x}$ becomes $R_{x}=0.157 / t$. The resultant resistance of the parallel resistance combination is

$$
R=\frac{R_{S} R_{X}}{R_{S}+R_{X}}=\frac{0.157 / t}{1+0.157 / t}
$$




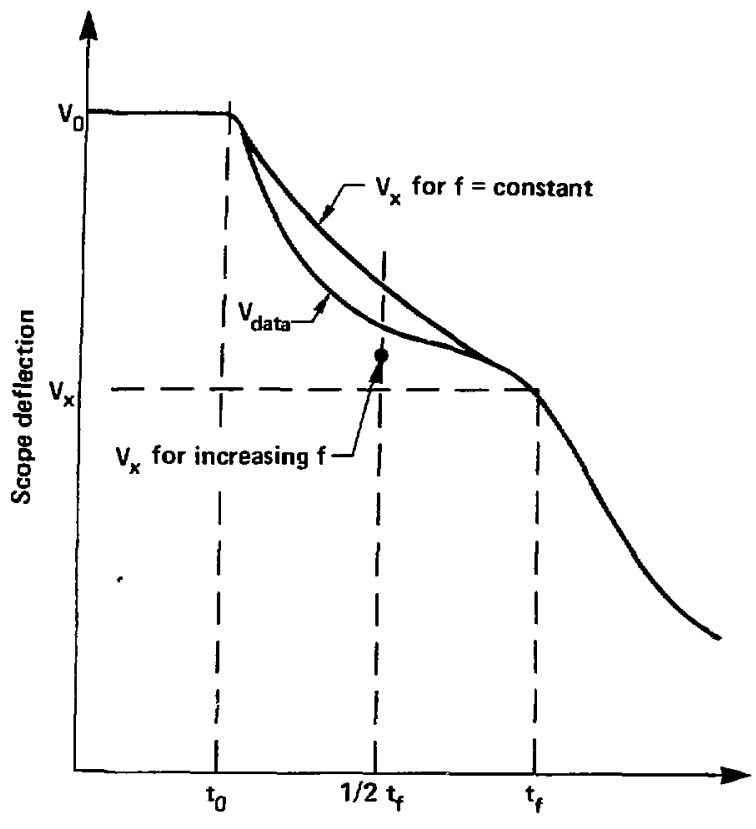

Time

Fig. C.1 Comparison of signal record with calculated shape assuming constant and reduced $f$.

This leads to the following expression for the scope deflection, $v_{x}$,

$$
v_{x}=\frac{v_{0}}{R_{C L}}\left[\frac{0.157 / t}{0.157 / t+1}\right] \text {, }
$$

where $v_{0}$ is the initial deflection and $R_{C L}$ is the resistance of the current limiters. If $V_{0} / R_{c L}$ is scaled to the initial deflection for $t<t_{0} \equiv 0$ then

$$
v_{x}=c_{1}\left[\frac{c_{2} / t}{c_{2} / t+1}\right] \text {. }
$$


The calculated signal shape is also shown in Fig. C.1. The disparity between the calculated signal shape and measured signal shape suggests that the assumption of a constant fringing-field correction is invalid. This seems reasonable since a greater departure from $f=1$ as $\mathrm{A} / \mathrm{l}$ decreises is to be expected.

Some preliminary measurements on the dependence of $f$ on the ratio $A / \ell$ were done for three values of $A / \ell(.01,0.1,10)$. These measurements gave a progressively smaller $f$ for decreasing $A / \ell(f \sim 1$ $\rightarrow 0.05)$.

The increase of $f$ from $f\left(t=t_{0}\right)$ to $f\left(t=t_{f}\right)$ is consistent with the observed trend of Fig. C.1. For half-exposure of the electrode surface, the ratio $\mathrm{A} / \mathrm{l}$ is $\mathbf{0 . 0 5}$. The measured fringing field correction for this ratio is 0.25 and corresponds to $t=1 / 2 t_{f}$. The deflection is also included in Fig. C.I and appears to be too small when compared with the data. This probably reflects the crude procedure in determining the geometry invoived in calculating $A / l$ and underscores the importance of implementing a better-defined geometry when the concern is accurate time-resolved measurements. 
Detatis of the sample cavity dimensions, baseplate and impactor densities and thicknesses, electrode materials, and cell constants are given in Table D.1. Uncertainties in the impactor and baseplate densities were typically $\pm 0.002 \mathrm{~g} / \mathrm{cm}^{3}$. Uncertainties in the target dimensions were approximately $0.002 \mathrm{~mm}$. Cell constant uncertainties were assigned $a$ value of $10 x$. Figure $D .1$ defines the $x_{j}$ symbols used in the table. For conductivity shots, $x_{5}$ is the distance between the electrode tip and baseplate. For shock velocity measurements, $x_{5}$ is the distance between the second pin plane and the baseplate.

Electrode separations were nominally $1 \mathrm{~mm}$ except for $\mathrm{CBZ}-10$, which were separated $3 \mathrm{~mm}$. Sulphur was added to CBZ-11 at a concentration of 0.15 percent by weight. 


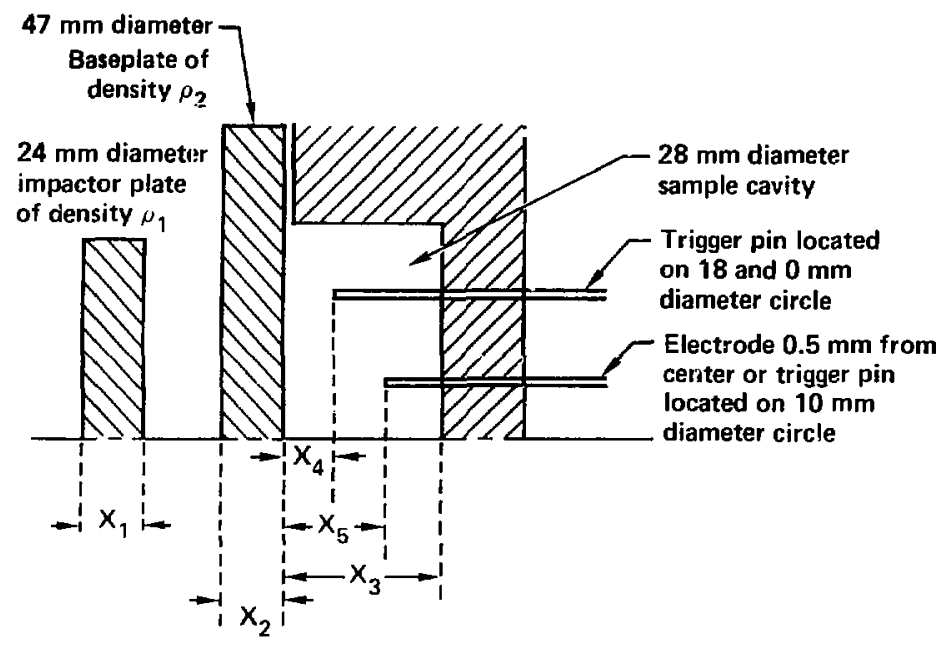

Fig. 0.1 Target dimensions. 
Table D.1 Baseplate and Impactor Densities, Target Dimensions, Electrode Materials, and Cell Constants.

\begin{tabular}{|c|c|c|c|c|c|c|c|c|c|}
\hline Shot & $\begin{array}{c}P_{1} \\
\left(g / \mathrm{cm}^{3}\right)\end{array}$ & $\left(\begin{array}{c}P_{2} \\
\left(g / \mathrm{cm}^{3}\right)\end{array}\right.$ & ${ }_{(m)}^{x_{1}}$ & $\begin{array}{c}x_{2} \\
(m m)\end{array}$ & $\begin{array}{c}x_{3} \\
(m m)\end{array}$ & $\begin{array}{c}x_{4} \\
(m m)\end{array}$ & $\underset{(m)}{x_{5}}$ & terial & $\begin{array}{l}\text { a) } \mathrm{F} \\
\left(\mathrm{cm}^{-1}\right)\end{array}$ \\
\hline $\begin{array}{r}\text { NC-3 } \\
6 \\
8 \\
9 \\
10 \\
11\end{array}$ & $\begin{array}{r}2.713 \\
2.712 \\
2.712 \\
2.713 \\
2.717 \\
16.566\end{array}$ & $\begin{array}{l}2.748 \\
2.748 \\
2.711 \\
2.745 \\
2.731 \\
2.710\end{array}$ & $\begin{array}{l}3.005 \\
3.008 \\
4.025 \\
4.004 \\
4.025 \\
2.068\end{array}$ & $\begin{array}{l}2.020 \\
2.015 \\
2.972 \\
2.00(\mathrm{~b}) \\
2.008 \\
2.008\end{array}$ & $\begin{array}{l}3.960 \\
4.401 \\
4.442 \\
5.07(\mathrm{~b}) \\
4.697 \\
4.694\end{array}$ & $\begin{array}{l}2.416 \\
2.368 \\
2.759 \\
1.411 \\
2.828 \\
2.734\end{array}$ & $\begin{array}{l}2.944 \\
3.347 \\
3.579 \\
2.705 \\
3.766 \\
3.636\end{array}$ & $\begin{array}{l}\text { SS } \\
\text { SS } \\
\text { SC } \\
\text { SC } \\
\text { SS } \\
\text { SC }\end{array}$ & $\begin{array}{l}2.0 \\
3.8 \\
4.2 \\
3.4 \\
3.7 \\
3.1\end{array}$ \\
\hline $\begin{array}{r}\mathrm{L} 02-\mathrm{C} 1 \\
2 \\
3 \\
5 \\
6\end{array}$ & $\begin{array}{l}2.713 \\
2.71(b) \\
2.713 \\
2.717 \\
2.717\end{array}$ & $\begin{array}{l}2.751 \\
2.745 \\
2.748 \\
2.731 \\
2.747\end{array}$ & $\begin{array}{l}3.920 \\
3.986 \\
3.990 \\
4.009 \\
4.012\end{array}$ & $\begin{array}{l}1.986 \\
2.00(b) \\
2.008 \\
2.022 \\
2.023\end{array}$ & $\begin{array}{l}5.446 \\
5.943 \\
5.286 \\
5.318 \\
5.468\end{array}$ & $\begin{array}{l}2.506 \\
2.527 \\
2.480 \\
2.886 \\
2.785\end{array}$ & $\begin{array}{l}4.397 \\
4.941 \\
4.319 \\
4.320 \\
4.431\end{array}$ & $\begin{array}{l}\text { SS } \\
\text { SS } \\
\text { SS } \\
\text { SS } \\
\text { SC }\end{array}$ & $\begin{array}{l}2.3 \\
5.5 \\
3.9 \\
2.5 \\
5.6\end{array}$ \\
\hline $\begin{array}{r}\text { C8Z-4 } \\
6 \\
7 \\
10 \\
11 \\
14 \\
15 \\
16\end{array}$ & $\begin{array}{r}2.698 \\
2.699 \\
2.699 \\
2.714 \\
2.713 \\
2.712 \\
16.687 \\
16.688\end{array}$ & $\begin{array}{l}2.706 \\
2.716 \\
2.710 \\
2.711 \\
2.710 \\
2.711 \\
2.712 \\
2.716\end{array}$ & $\begin{array}{l}4.037 \\
4.006 \\
4.029 \\
3.956 \\
3.956 \\
4.026 \\
1.955 \\
1.955\end{array}$ & $\begin{array}{l}2.044 \\
2.001 \\
3.070 \\
1.976 \\
2.014 \\
1.981 \\
3.018 \\
3.004\end{array}$ & $\begin{array}{l}5.232 \\
5.080 \\
4.534 \\
4.567 \\
4.607 \\
4.406 \\
4.229 \\
4.438\end{array}$ & $\begin{array}{l}1.668 \\
2.951 \\
2.951 \\
1.624 \\
1.651 \\
0.281 \\
0.028 \\
2.494\end{array}$ & $\begin{array}{l}4.252 \\
4.163 \\
3.506 \\
3.577 \\
3.783 \\
3.426 \\
3.472 \\
1.564\end{array}$ & $\begin{array}{l}\text { SS } \\
\text { SS } \\
\text { SS } \\
\text { SS } \\
\text { SS } \\
5 C \\
\text { SC } \\
\text { SC }\end{array}$ & $\begin{array}{l}4.7 \\
5.2 \\
3.3 \\
7.4 \\
4.4 \\
3.8 \\
4.6 \\
3.3\end{array}$ \\
\hline $\begin{array}{r}\text { L2C4H8EQTB } \\
2 \\
4\end{array}$ & $\begin{array}{r}2.715 \\
16.680 \\
2.714\end{array}$ & $\begin{array}{l}2.716 \\
2.700 \\
2.699\end{array}$ & $\begin{array}{l}3.00(b) \\
1.576 \\
3.015\end{array}$ & $\begin{array}{l}2.023 \\
2.010 \\
2.031\end{array}$ & $\begin{array}{l}6.360 \\
6.446 \\
6.393\end{array}$ & $\begin{array}{l}0.149 \\
0.360 \\
0.305\end{array}$ & $\begin{array}{l}1.666 \\
2.603 \\
2.542\end{array}$ & $\begin{array}{l}-- \\
-- \\
-\end{array}$ & $\begin{array}{l}-- \\
-- \\
--\end{array}$ \\
\hline
\end{tabular}

(a) $s s=$ platinum-plated stainless steel and $s c=$ platinum-plated soft copper

(b) Prectse measurements not recorded, so nominal values given. 


\section{REFERENCES}

1. Finger, M., Lee, E., Helm, F. H., Hayes, B., Hornig, H., McGuire, R., Kahara, M., and Guidry, M., "The Effect of Elenental Composition on the Detonation Behavior of Explosives," Proc. Sixth Symp. on Detonation, ACr-221, Office of Naval Research - Dept. of the Navy (1976).

2. Ree, F. H., "Super Critical Fluid Phase Separations: Implications for Detonation Properties of Condensed Explosives," Lawrence Livermore National Laboratory, UCRL-93929 (1986).

3. Ree, F. H., "Statistical Mechanica; Theory of Chemically Reacting Multiphase Mixtures: Application to the Detonation Properties of PEIN." J. Chem. Phys., 81, 1251 (1984).

4. Stevenson, D. J., "High Pressure Physics and Chemistry in Giant Planets and their Satellites," J. de. Physique, 45, C8-97 (1984).

5. Stevenson, D. J., "Condensed Matter Physics of Planets: Puzzles, Progress and Predictions," Mat. Res. Soc. Symp. 22, 357 (1984).

6. Nellis, W. J., Holmes, N. C., Mitchell, A. C., and van Thie1, M., "Phase Transition tn Fluid Nitrogen at High Densities and Temper.itures," Phys. Rev. Lett. 53, 1661 (1984). 
7. Mellis, W. J. and Mitchell, A. C., "Shock Compression of Liquid Argon, Nitrogen, and Oxygen to $90 \mathrm{GPa}, " \mathrm{~J}$. Chem. Phys. $\underline{73}, 6137$ (1980).

8. Zabarev, V. N. and Telegin, 6. S., Akad. Nauk SSSR, 142, 309 (1962) [Sov. Phys. Dok I. I, 34 (1962)].

9. Ross, M. and Ree, F. H., "Repulsive Forces of Simple Molecular and Mixtures at High Density and Temperature," J. Chem. Phys. 73,6146 (1980).

10. LeSar, R., Ekberg, S. A., Jones, L. H., M111s, R. L., Schwelbe, L. A., and Schifer1, D., "Raman Spectroscopy of Solid Nitrogen up to 374 Kbar," Solfd State Commun. 32, 131 (1979).

11. Retchlin, R., Schiferl, D., Martin, S., Vanderborgh, C., and Mills, R. L., "Optical Studies of Nitrogen to $130 \mathrm{GPa,"} \mathrm{Lawrence}$ Livermore National Laboratory, UCRL-93168 (1985).

12. Radousky, H. B., Nellis, W. J., Ross, M., Hamilton, D. C., and Mitche11, A. C., "Molecular Dissociation and Shock-Induced Cooling In Flutd Nitrogen at High Densities and Temperature," Lawrence Livermore National Laboratory, UCRL 94515 (1986).

13. Ross, M., "The Dissociation of Dense Liquid Nitrogen," Lawrence Livermore Mational Laboratory, UCRL 95234 (1986). 
14. McMahan, A. K. and LeSar, R., "Pressure Dissociation of Nitrogen Under 1 Mbar," Phys. Rev. Lett. 55, 1929 (1985).

15. Wackerle, J., Seita, W. L., and Jamieson, J. C., in Behavior of Dense Media under High Dynamic Pressure (Gordon and Breach, New York, 1968), p. 85.

16. Nellis, W. J., Ree, F. H., Trainor, R. J., Mitche11, A. C., and Boslough, M. B., "Equation of State and Optical Luminosity of Benzene, Polybutene, and Polyethylene Shocked to $210 \mathrm{GPa}$, Lawrence Livermore National Laboratory, UCRL-89711 (1983).

17. Dick, R. D., "Shock Waye Compression of Benzene, Carbon Disulfide, Carbon Tetrachloride, anu Liquid Nitrogen," J. Chem. Phys. $\underline{52}, 6021$ (1970).

18. Yakushev, O. B., Yakushev, V. V., and Oremin, A. N., "The Opacity Mechanism of Shock-Compressed Organic Liquids," High-Temp.-High Press. 3, 261 (1971).

19. Ershov, A. P., "Ionization During Detonation of Solid Explosives," Combustion Explosion and Shockwaves, 11, 798 (1975).

20. Al'tshuler, L. V., Kuleshova, L. V., and Pavlovski, M. N., "The Dynamic Compressibility, Equation of State, and Electrical Conductivity of Sodium Chloride at High Pressures," Sov. Phys. JETP 12. 10 (1961). 
27. Balchan, A. S. and Drickamer, H. G., "Effect of Pressure on the Resistance of Iodine and Selenium," J. Chem. Phys. 34, 1948 (1961).

22. Kormer, S. B., Sinitsyn, M. V., Kirillov, G. A., and Popova, L. T., "An Experimental Determination of the Light Absorption Coefficient in Shock-Compressed $\mathrm{NaCl}$. The Absorption and Conductivity Mechanism," Sov. Phys. JETP 22, 97 (1966).

23. Ahrens, T. J., "High-Pressure Electrical Behavior and Equation of State of Magnesium 0xide from Shock Wave Measurements," J. Appl. Phys. 37,2532 (1966).

24. Hamann, S. D. and Linton, M., "Electrical Conductivity of water in Shock Compression." Trans. Faraday Soc. 62, 2234 (1966).

25. Mitche11, A. C. and Keller, R. N., "Accurate Measurement of the Electrical Conductivity of Shocked Fluids," Lawrence Livermore National Laboratory, UCRL-70136 (1967).

26. Kaleshova, L. V., "Electrical Conductivity of Boron Nitride, Potassium Chloride, and Polytetrafluoroethylene Behind a Shock-Wave Front." Sov. Phys. Solid State 11, 886 (1969).

27. Yakushev, V. V. and Oremin, A. N., "Electrochemical Effecis During Shock Compression of Dielectrics. Mechanism of Electrical Conduction in Shack Compressed Liquids," Russian Journal of Phys. Chem. 45. $50(1971)$. 
28. Piche, E. and Rubin, G. A., "The Effect of Pressure on the Electrical Conductivity of Sapphire," Canadian J. Phys. 51, 9 (1973) .

29. Gatilov, L. A. and Kuleshova, L. V., "Heasurements of High Electrical Conductivity in Shock-Compressed Dielectrics, "Soviet J. Appl. Mechanics and Tech. Phys. 22, 114 (1981).

30. Gatilov, L. A. and Kuleshova, L. V., "Electrical Conductivity of Cesiun Iodine Behind a Shock-Wave Front at Pressures up to 100 GPa," Sor. Phys. Solid State 23, 1663 (1981).

31. Champion, A. R., "Effect of Shock Compression on Electrical Resistivity of Three Polymers," J. Appl. Phys. 43, 2216 (1972).

32. Solids Under Pressure, ed. by Paul, W. and Warschauer, D. M., (McGraw-Hill, Inc., New York, 1963).

33. Fritzsche, H., in Physics of Solids at High Pressures, ed. by Tomizuka, C. T. and Emrick, R. M. (Academic Press, New York, 1965), p. 184 .

34. Drickamer, H. G. and Frank, C. W., in Electronic Transitions and the High Pressure Chemistry and Physics of Solids (Chapman and Ha 11, London, 1973). 
35. Zallen, R., Physics of Amorphous Solids (John Wil ley and Sons, New York, 1983).

36. Harrison, W. A., Electronic structure and the Properties of Solids (W. H. Freeman and Co., San Francisco, 1980).

37. Ziman, J. M., Models of Disorder (Cambridge University Press, Cambridge, 1979).

38. Mott, N. F., Metal-Insulator Transittons (Taylor and Franc is LTD, London, 1974), Chapter 1.

39. Mott, N. F. and Twose, W. D., "The Theory of Impurity Conduction," Adv. Phys. 10, 107 (1961).

40. Mott, N. F., "Conduction in Non-Crystalline Systems: I. Localized Electronic States in Disordered Systems," Phil. Mag. 17, 1259 (1968).

41. Hott, N. F., "Conduction in Non-Crystalline Systems: II. The Meta 1-Insulator Transition in a Random Array of Centres," Phil. Mag. 17, 1269 (1968).

42. Mott, N. F., "Conduction in Non-Crystalline Materials:

III. Localized States in a Pseudo Gap and Near Extremities of Conduction and Valence Bands," Phi1. Mag. 19, 835 (1969). 
43. Mott, N. F., "Conduction in Non-Crystalline Systems: IV. Anderson Localization in a Disordered Lattice," Phil. Mag. 22, 7 (1970).

44. Spear, W. E., "Electronic Transport and Localization in Low Mobility Solids and Liquids," J. Non-Cryst. Solids, 523 (1974).

45. Hulls, K. and McMillan, P. W., "Amorphous Semiconductors: A Review of Current Theories," J. Phys. D: App 1. Phys. $\underline{5}, 865$ (1972).

46. Boer, K. W., "The Electrical Conduction Mechanism in Highly Disordered Semiconductors," J. Non-Cryst. Solids 2, 444 (1970).

47. Davis, E. A. and Shaw, R. F., "Characteristic Phenomena in norphous Semiconductors," J. Non-Cryst. Solids 2, 406 (1970).

48. Cohen, M. H., "Review of the Theory of Amorphous Semiconductors," J. Non-Cryst. Solids 4,391 (1970).

49. Cohen, H. H., "Electronic Structure and Transport in Covalent Amorphous Semiconducting Alloys," J. Non-Cryst. Solids, 2, 432 (1970).

50. Ree, F. H. and Winter, N. W., "Ab Initio and Gordon-Kim Intermolecular Potentials for Two Nitrogen Molecules," J. Chem. Phys. $\underline{73}, 322$ (1980). 
51. Hequarrie, D. A., Statistical Mechanics, (Harper and Row, New York, 1976).

52. Harsen, J. P. and McDona 1d, I. R. Theory of Simple Liquids, (Academic Press, London, 1975).

53. Egelstaff, P. A., An Introduction to the Liquid state, (Academic Press, London, 1967).

54. Ross, M., "The Repulsive Forces in Dense Argon," 3. Chem. Phys. 73, $4445(7980)$

55. Ross, M., "A High-Density Fluid Perturbation Theory Based on an Inverse 12th-Power Hard-Sphere Reference System," J. Chem. Phys., 71. 1567 (1979).

56. Ree, F. H., "Systematics of High-Pressure and High-Temperature Behavior of Hydrocarbons," J. Chem. Phys., 70, 974 (1979).

57. Hayes, D. B.. "Introduction to Stress Wave Phenomena," Sandia Laboratories, Albuquerque, SLA-73-0801 (August 1973).

58. Holian, B. L., Hoover, W. G., Moran, B., and Straub, G. K., -Shock -wave Structure via Nonequilibrium Molecular Dynamics and Navier-Stokes Continuum Mechanics," Phys. Rev. A, 22, 2798 (1980). 
59. Karpman, V. I., Man-linear Waves in Dispersive Media, (Pergamon Press, 0xford, 1975).

60. Holian, B. L., "Simulation of Vibrational Relaxation in Dense Molecular Fluids. 1. Methods," Chem. Phys. B4, 3138 (1986). See earlier work in, Johnson, J. D., Shaw, M. S., and Holian, B. L., "The Thermodynamics of Dense Fluid Nitrogen by Molecular Dynanics." J. Chem. Phys, B0, 1279 (1984).

61. Zel'dovich, Ya. B. and Raizer, Yu. P., Physics of Shock Waves and High-Temperature Hydrodynamic Phenomena, (Academic Press, Hew York, 1966).

62. Shapiro, Ascher H., The Dynamics and Thermodynamics of Compressible Fluid Flow, (The Ronald Press Co., New York, 1953).

63. Al'thshuler, L. V., Kormer, S. B., Brashrik, M. I., Vladimirov, L. A., Speranskaya, M. P., and Fatikov, A. I., "The Isentropic Compressibility of Aluminum, Copper, Lead and Iron at High Pressures," Soviet Physics JETP, 11, 766 (1960).

64. Mitche11, A. C. and Nellis, W. 3., "Diagnostic System of the Lawrence Livermore National Laboratory Two-Stage Gas Gun," Rev. Sci. Instrum. 52, 347 (7981). 
65. Mitche 11, A. C. and Nellis, W. J., "Equation of State and Electrical Conductivity of Water and Ammonia Shocked to the $100 \mathrm{GPa}$ Pressure Range," J. Chem. Phys. $\underline{76}, 6273$ (1982).

66. Mitchell, A. C. and Nellis, W. J., "Shock Compression of Aluminum, Copper and Tantalum," J. App 1. Phys. 52, 3363 (1981).

67. Nellis, W. J.. "Shocked Fluids at High-Densities and Temperatures," Asay, J. R., Graham, R. A. and Straub, G. K., (ed.), Shock Waves in Condensed Matter. (Elsevier Scienc: Publishers B.V., 1984).

68. Isbell, W. M., Shipman, F. H., and Jones, A. H., "Use of a Light-gas Gun in Studying Material Behavior at Megabar Pressures," in Behavior of Dense Media Under High Dynamic Pressures, Symposium H.D.P., Paris 1967 (Gordon and Breach, New York and Dunod, Paris, 1968).

69. Woodruff, J. P., Kovec Users Manual, Lawrence Livermore National Laboratory, UCID-17306 (1976) and Wilkins, M. L., "Calculation of Elastic-Plastic Flow, "Lawrence Livermore National Laboratory, UCRL 7322, Rev. 1 (1969).

70. Yakushev, V. V., "Electrical Measurements in a Oynamic Experiment," Combustion, Explosion and Shockwaves, 14, 131 (1978). 
71. Mitche11, A. C. and Keeler, R. N., "Technique for Accurate Measurement of the Electrical Conductivity of Shocked Fluids, "Rev. Sci. Instrum., 39, 513 (1968).

72. Brish, A. A., Tavasov, M. S., and Tsukerman, V. A., "Electrical Conductivity of Dielectrics in Strong Shock Waves," Sov. Phys. JETP, 11, 15 (1960).

73. Brish, A. A., Tavasov, M. S., and Tsukerman, V. A., Electrical Conductivity of the Explosion Products of Condensed Explosives," Sov. Phys. JETP, 37, 1095, (1960).

74. Matassov, 6., "The Electrical Conductivity of Iron Silicon Alloys at High Pressures and the Earths Core," Ph.D Thesis, Lawrence Livermore Nationa 1 Laboratory, UCRL-52322, (1977).

75. Vargaftik, H. B., Tables on the Thermophysical Properties of Liquids and Gases, (John Wiley \& Sons, New York, 1975).

76. Jacobsen, R. T., Stewart, R. 2., McCarty, R. D., and Hanley, H. J. M., Thermophysical Properties of Nitrogen From the Fusion Line to $3500 \mathrm{R}$ for Pressures to 150,000 PSIA, National Bureau of Standards, Technical Note $648,1973$.

77. Chao, J., Key Chemicals Data Books - Benzene (Thermodynamics Research Center, Texas Engr. Experiment Station, Texas A\&H University, 1978). 
78. Hoshino, H., Schmutzler, R. W., Warren, W. W., and Hense 1, F., "The Electrical Conductivity of Fluid Selenium Up to Super Critical Temperatures and Pressures," Ph11. Mag. 33, 255 (1976).

79. Renkert, H., Hensel, F., and Franck, E. U., "The Electrical Conductivity and the Metal-Nonmetal Transition in Dense Cesium Vapor." J. Non-Cryst. Solids, 4, 180 (1970).

80. Hense 1, F., "Optical Absorption in Dense Gaseous Hercury," J. Non-Cryst. Solids, 4. $179(1970)$.

81. Mott, N. F. and Davis, E. A., Electronic Processes in Non-Crystalline Materials, (Clarendon Press, Oxford, 1979).

82. Elliott, S. R., Physics of Amorphous Materials (Longman, London, 1983).

83. Ross, R. G. and Greenwood, D. A., "Liquid Metals and Vapors Under High Pressure," Progress in Materials Science, ed. Chalmers, Bruce and Hame-Rothery, W., Vol. 14, p. 215, (Pergamon Press, Oxford).

84. Seager, C. H. and Pike, G. E., "Percolation and Conductivity: A Computer Study. II," Phys. Rev. B, 10, 1435 (1974).

85. Abeles, B., Sheng., P., Coutts, M. O., and Arie, Y., "Structural and Electrical Properties of Granular Metal Films," Adv. Phys. 24 , 407 (1975). 
86. Malliaris, A., and Turner, D. T., "Influence of Particle Size on the Electrical Resistivity of Compacted Mixtures of Polymeric and Metallic Powders," J. App1. Phys. 42, 614 (1971).

87. Gurland, J., "An Estimate of Contact and Continuity of Dispersions in Opaque Samples," Trans. Met. Soc. AIME, 236, 642 (1966).

8B. Physical Properties of Amorphous Materials (Ed. Aider, 0., Schwarts, B. B., Steele, M. C., Plenum Press, New York, 1985).

B9. Beers, Y., Introduction to the Theory of Error, (Addison Wes ley, Reading, MA, 1957).

90. Handbook of Chemistry and Physics, 44th Ed. (Chemical Rubber Publishing Co., Cleveland, $\mathrm{OH}, 1962$ ). 